\title{
GRAVITY WAVE DYNAMICS AND EFFECTS IN THE MIDDLE ATMOSPHERE
}

\author{
David C. Fritts and M. Joan Alexander \\ Colorado Research Associates Division \\ Northwest Research Associates \\ Boulder, Colorado, USA
}

Received 6 July 2001; revised 29 August 2002; accepted 6 November 2002; published 16 April 2003.

[1] Atmospheric gravity waves have been a subject of intense research activity in recent years because of their myriad effects and their major contributions to atmospheric circulation, structure, and variability. Apart from occasionally strong lower-atmospheric effects, the major wave influences occur in the middle atmosphere, between $\sim 10$ and $110 \mathrm{~km}$ altitudes because of decreasing density and increasing wave amplitudes with altitude. Theoretical, numerical, and observational studies have advanced our understanding of gravity waves on many fronts since the review by Fritts [1984a]; the present review will focus on these more recent contributions. Progress includes a better appreciation of gravity wave sources and characteristics, the evolution of the gravity wave spectrum with altitude and with variations of wind and stability, the character and implications of observed climatologies, and the wave interaction and instability processes that constrain wave amplitudes and spectral shape. Recent studies have also expanded dramatically our understanding of gravity wave influences on the large-scale circulation and the thermal and constituent structures of the middle atmosphere. These advances have led to a number of parameterizations of gravity wave effects which are enabling ever more realistic descriptions of gravity wave forcing in large-scale models. There remain, nevertheless, a number of areas in which further progress is needed in refining our understanding of and our ability to describe and predict gravity wave influences in the middle atmosphere. Our view of these unknowns and needs is also offered. INDEX TERMS: 3319 Meteorology and Atmospheric Dynamics: General circulation; 3334 Meteorology and Atmospheric Dynamics: Middle atmosphere dynamics (0341, 0342); 3379 Meteorology and Atmospheric Dynamics: Turbulence; 3384 Meteorology and Atmospheric Dynamics: Waves and tides; KEYWORDS: gravity waves; middle atmosphere dynamics; wavemean flow interactions; wave-wave interactions; gravity wave instability; turbulence

Citation: Fritts, D. C., and M. J. Alexander, Gravity wave dynamics and effects in the middle atmosphere, Rev. Geophys., 41(1), 1003, doi:10.1029/2001RG000106, 2003.

\section{INTRODUCTION}

[2] Considerable progress has occurred in our understanding of middle atmosphere gravity waves in the four decades since the pioneering study by Hines [1960]. Indeed, interest in the field continues to expand as the many effects of gravity waves become better understood. Early results were reviewed extensively by Fritts [1984a], while subsequent reviews addressed specific aspects of gravity wave instability [Fritts and Rastogi, 1985], saturation [Dunkerton, 1989; Fritts, 1989], or climatologies and effects [Vincent, 1984a; Schoeberl, 1987; Hamilton, 1996; McLandress, 1998]. Arguably, the greatest quantitative advances in our understanding have been the most recent and have accompanied equally dramatic advances in observational and computational techniques and capabilities. This review will span middle atmosphere gravity wave dynamics and effects but will emphasize results subsequent to the review by Fritts [1984a].
[3] In retrospect, our understanding of middle atmosphere gravity waves in 1984 was quite limited. We had recognized the role of gravity waves in transporting energy and momentum, in contributing turbulence and mixing, and in influencing the mean circulation and thermal structure of the middle atmosphere, based on limited theoretical, numerical, and observational studies [Lindzen, 1981; Holton, 1982, 1983; Vincent and Reid, 1983]. However, we had little or no understanding of the characteristics of the gravity wave spectrum and of its behavior with altitude, of the variability imposed by sources and variable mean and low-frequency motions, of the global climatology of gravity waves and their effects, or of the processes and interactions that constrain wave amplitudes, effect spectral energy transfers, and account for turbulence, fluxes, and mixing. We also had explored only the most rudimentary schemes for describing gravity wave effects in large-scale models [Lindzen, 1981; Holton, 1982; Matsuno, 1982; Dunkerton, 1982a] and had no appreciation for the rich range of 
behaviors accompanying gravity waves throughout the middle atmosphere.

[4] More recent studies have yielded a more detailed understanding of middle atmosphere gravity waves on many fronts. In situ, ground-based, and space-based observational studies have contributed greatly to our knowledge of gravity wave scales, amplitudes, fluxes, and spectra. They have also addressed instability dynamics, vertical propagation, variations with altitude, and seasonal and geographic variability. Recent theoretical and numerical studies have addressed source characteristics and scales, spectral character, evolution, and energy transfers, instability dynamics, wave-wave and wavemean flow interactions, and the implications of various parameterizations for atmospheric circulation and structure.

[5] Our purpose here is to review the contributions since 1984 that have led to our more advanced understanding of middle atmosphere gravity waves and their effects. In doing so, it will be impossible to cite all of the work that has been published. Instead, we will restrict ourselves to what we consider to be the more significant contributions in each area. We also will note, on occasion, where errors in interpretation have occurred and where debate over observational or theoretical implications continues to allow the reader to steer clear of mistakes and identify topics of current interest.

[6] We begin by reviewing the linear inviscid theory of gravity wave propagation as well as departures due to nonlinearity, dissipation via wave breaking, transience, localization, and variable environments, in section 2 . Sources, propagation, and scales relevant to the middle atmosphere will be addressed in section 3 . Spectral character and evolution, including evidence for and against universality, are reviewed in section 4 . Section 5 summarizes what is known of gravity wave climatologies and their implications for sources and effects. Gravity wave interactions, instability dynamics, and wave-breaking or saturation processes are examined in section 6. Sections 7 and 8 describe efforts to parameterize gravity wave effects to date and their implementation and effects on the large-scale circulation and structure of the middle atmosphere. Our summary and recommendations are presented in section 9.

\section{EQUATIONS OF MOTION}

[7] Atmospheric gravity waves can frequently be described with a simple linear theory that treats them as small departures from a stably stratified background state varying only in the vertical. The restoring force for gravity wave oscillations is the buoyancy that results from the adiabatic displacements of air parcels characteristic of these disturbances. Here we outline a general gravity wave solution to the linearized forms of the fundamental conservation equations. We then discuss the simpler forms of the dispersion and polarization relations that result when limited portions of the possible gravity wave spectrum are considered.

\subsection{Linear Theory}

[8] We begin with the fundamental fluid equations in Cartesian coordinates $(x, y, z)$ that follow from conservation of momentum, mass, and energy [e.g., Holton, 1992]:

$$
\begin{gathered}
\frac{d u}{d t}-f v+\frac{1}{\rho} \frac{\partial p}{\partial x}=X, \\
\frac{d v}{d t}+f u+\frac{1}{\rho} \frac{\partial p}{\partial y}=Y, \\
\frac{d w}{d t}+\frac{1}{\rho} \frac{\partial p}{\partial z}+g=0, \\
\frac{1}{\rho} \frac{d \rho}{d t}+\frac{\partial u}{\partial x}+\frac{\partial v}{\partial y}+\frac{\partial w}{\partial z}=0, \\
\frac{d \theta}{d t}=Q,
\end{gathered}
$$

where $d / d t$ represents a total, or advective, derivative; $(u, v, w)$ is the fluid velocity vector; and the terms $X, Y$, and $Q$ represent unspecified forcings that could include wave-driven forces and diffusive mixing effects accompanying wave dissipation. The remaining symbols have the usual meanings: $p$ is pressure, $\rho$ is density ( $\rho$ $=\rho_{0} \exp \left[-\left(z-z_{0}\right) / H\right]$ with $\rho_{0}=\rho\left(z_{0}\right)$ the density at reference level $z_{0}$ and $H$ the scale height), and $f=2 \Omega \sin \phi$ is the Coriolis parameter (where $\Omega$ is the Earth rotation rate and $\phi$ is latitude). These five equations plus the definition of potential temperature $\theta$,

$$
\theta=\frac{p}{\rho R}\left(\frac{p_{0}}{p}\right)^{\kappa},
$$

define a complete set describing inviscid fluid motions. Here $p_{0}=p\left(z_{0}\right), R$ is the ideal gas constant, and $\kappa$ $=c_{p} / c_{v}$ is the ratio of specific heats at constant pressure and constant volume. Here $\theta$ represents the temperature a parcel of air would have if lowered adiabatically from $p$ to $p_{0}$.

[9] The unforced forms of equations (1)-(6) linearized about a horizontally uniform hydrostatic basic state with background wind $(\bar{u}, \bar{v}, 0)$, potential temperature $\bar{\theta}$, pressure $\bar{p}$, and density $\bar{\rho}$ varying only in $z$ are

$$
\begin{gathered}
\frac{D u^{\prime}}{D t}+w^{\prime} \frac{\partial \bar{u}}{\partial z}-f v^{\prime}+\frac{\partial}{\partial x}\left(\frac{p^{\prime}}{\bar{\rho}}\right)=0, \\
\frac{D v^{\prime}}{D t}+w^{\prime} \frac{\partial \bar{v}}{\partial z}+f u^{\prime}+\frac{\partial}{\partial y}\left(\frac{p^{\prime}}{\bar{\rho}}\right)=0, \\
\frac{D w^{\prime}}{D t}+\frac{\partial}{\partial z}\left(\frac{p^{\prime}}{\bar{\rho}}\right)-\frac{1}{H}\left(\frac{p^{\prime}}{\bar{\rho}}\right)+g \frac{\rho^{\prime}}{\bar{\rho}}=0, \\
\frac{D}{D t}\left(\frac{\theta^{\prime}}{\bar{\theta}}\right)+w^{\prime} \frac{N^{2}}{g}=0,
\end{gathered}
$$




$$
\begin{gathered}
\frac{D}{D t}\left(\frac{\rho^{\prime}}{\bar{\rho}}\right)+\frac{\partial u^{\prime}}{\partial x}+\frac{\partial v^{\prime}}{\partial y}+\frac{\partial w^{\prime}}{\partial z}-\frac{w^{\prime}}{H}=0, \\
\frac{\theta^{\prime}}{\bar{\theta}}=\frac{1}{c_{s}^{2}}\left(\frac{p^{\prime}}{\bar{\rho}}\right)-\frac{\rho^{\prime}}{\bar{\rho}} .
\end{gathered}
$$

Here the derivative $D / D t$ is the linearized form of the time derivative,

$$
\frac{D}{D t}=\frac{\partial}{\partial t}+\bar{u} \frac{\partial}{\partial x}+\bar{v} \frac{\partial}{\partial y},
$$

primed quantities are perturbations to the background state, and $N=(g \partial \ln \theta / \partial z)^{1 / 2}$ is the buoyancy frequency.

[10] We now neglect the background shear terms in equations (7) and (8) by assuming $(\bar{u}, \bar{v})$ and $N$ vary only slowly over a wave cycle in the vertical (the WKB approximation [e.g., Gill, 1982]). We also assume the gravity wave solutions have the form

$$
\begin{aligned}
\left(u^{\prime}, v^{\prime}, w^{\prime},\right. & \left.\frac{\theta^{\prime}}{\bar{\theta}}, \frac{p^{\prime}}{\bar{\rho}}, \frac{\rho^{\prime}}{\bar{\rho}}\right)=(\tilde{u}, \tilde{v}, \tilde{w}, \tilde{\theta}, \tilde{p}, \tilde{\rho}) \\
& \cdot \exp \left[i(k x+l y+m z-\omega t)+\frac{z}{2 H}\right] .
\end{aligned}
$$

These describe a monochromatic wave perturbation with wave number components $(k, l, m)$ and groundrelative (Eulerian) frequency $\omega$. Substitution into equations (7)-(12) yields a set of six algebraic equations for $(\tilde{u}, \tilde{v}, \tilde{w}, \tilde{\theta}, \tilde{p}, \tilde{\rho})$ :

$$
\begin{gathered}
-i \hat{\omega} \tilde{u}-f \tilde{v}+i k \tilde{p}=0, \\
-i \hat{\omega} \tilde{v}+f \tilde{u}+i l \tilde{p}=0, \\
-i \hat{\omega} \tilde{w}+\left(i m-\frac{1}{2 H}\right) \tilde{p}=-g \tilde{\rho}, \\
-i \hat{\omega} \tilde{\theta}+\left(N^{2} / g\right) \tilde{w}=0, \\
-i \hat{\omega} \tilde{\rho}+i k \tilde{u}+i l \tilde{v}+\left(i m-\frac{1}{2 H}\right) \tilde{w}=0 \\
\tilde{\theta}=\tilde{p} / c_{s}^{2}-\tilde{\rho},
\end{gathered}
$$

where $\hat{\omega}=\omega-k \bar{u}-l \bar{v}$ is called the intrinsic frequency, i.e., the frequency that would be observed in a frame of reference moving with the background wind $(\bar{u}, \bar{v})$. The above equations can be combined to form a single equation for the perturbation vertical velocity amplitude. Demanding the imaginary coefficients of this equation go to zero gives,

$$
\frac{g}{c_{s}^{2}}=\frac{1}{H}-\frac{N^{2}}{g}
$$

$$
\begin{array}{r}
\hat{\omega}^{2}\left(k^{2}+l^{2}+m^{2}+\frac{1}{4 H^{2}}-\frac{\left(\hat{\omega}^{2}-f^{2}\right)}{c_{s}^{2}}\right)=N^{2}\left(k^{2}+l^{2}\right) \\
+f^{2}\left(m^{2}+\frac{1}{4 H^{2}}\right) .
\end{array}
$$

The second equation is fourth-order in $\hat{\omega}$ and supports both acoustic and gravity waves. By letting the sound speed $c_{s} \rightarrow \infty$ we retain the compressibility term related to the background density gradient and obtain the gravity wave dispersion relation,

$$
\hat{\omega}^{2}=\frac{N^{2}\left(k^{2}+l^{2}\right)+f^{2}\left(m^{2}+\frac{1}{4 H^{2}}\right)}{k^{2}+l^{2}+m^{2}+\frac{1}{4 H^{2}}},
$$

or alternatively for the vertical wave number as

$$
m^{2}=\frac{\left(k^{2}+l^{2}\right)\left(N^{2}-\hat{\omega}^{2}\right)}{\left(\hat{\omega}^{2}-f^{2}\right)}-\frac{1}{4 H^{2}} .
$$

The dispersion relation relates the wave frequency to the wave's spatial characteristics (wave numbers) and to the background atmosphere properties $N$ and $(\bar{u}, \bar{v})$. The gravity wave solutions above admit waves with a broad range of properties. For vertically propagating waves, $(k, l, m)$ are real, and the intrinsic frequency is confined to the range $N>\hat{\omega}>|f|$. All parts of this spectrum can be important to middleatmosphere dynamics. Because of this wide range of properties and because the intrinsic frequency and vertical wave number can vary strongly as a wave propagates vertically through background shear and stability gradients, models describing their propagation across a wide range of conditions may need to retain all terms in equation (23) in their analysis. Simpler forms representing waves in certain limited regions of this spectral space, however, provide considerable insight and will be summarized below.

[11] The wave group velocity describes energy transport and wave packet propagation and is written as

$$
\begin{aligned}
&\left(c_{g x}, c_{g y}, c_{g z}\right)=\left(\frac{\partial \omega}{\partial k}, \frac{\partial \omega}{\partial l}, \frac{\partial \omega}{\partial m}\right)=(\bar{u}, \bar{v}, 0) \\
&+\frac{\left[k\left(N^{2}-\hat{\omega}^{2}\right), l\left(N^{2}-\hat{\omega}^{2}\right),-m\left(\hat{\omega}^{2}-f^{2}\right)\right]}{\hat{\omega}\left(k^{2}+l^{2}+m^{2}+\frac{1}{4 H^{2}}\right)} .
\end{aligned}
$$

Our sign convention here assumes that $\hat{\omega}$ is positive definite. The vector $(k, l)$ defines the direction of horizontal propagation. Then $m$ is negative for upward group velocity and positive for downward. Thus, for eastward $(k>0)$ and upward $(m<0)$ energy propagation relative to the wind, intrinsic phase speeds are eastward $\left(\hat{c}_{x}=\hat{\omega} / k\right)$ and downward $\left(\hat{c}_{z}=\hat{\omega} / m\right)$ relative to the wind. Note that the phase speed is not a vector quantity, although wave phase propagation has a direction given by the vector $(k, l, m)$. 
[12] Polarization relations relating perturbation amplitudes of the different variables to one another can also be derived from equations (15)-(20). Some useful examples are

$$
\begin{gathered}
\tilde{u}=\left(\frac{i \hat{\omega} k-f l}{i \hat{\omega} l+f k}\right) \tilde{v}, \\
\tilde{p}=\left(\frac{\hat{\omega}^{2}-f^{2}}{\hat{\omega} k+i f l}\right) \tilde{u}=\left(\frac{\hat{\omega}^{2}-f^{2}}{\hat{\omega} l-i f k}\right) \tilde{v}, \\
\tilde{w}=\frac{\left(m-\frac{i}{2 H}\right) \hat{\omega}}{N^{2}-\hat{\omega}^{2}} \tilde{p},
\end{gathered}
$$

and equation (18).

\subsubsection{High-Frequency Waves: $\hat{\omega} \gg f$}

[13] The dispersion relation (23) is derived by neglecting acoustic wave solutions, but the terms proportional to $1 / 4 H^{2}$ retain one of the larger compressibility effects associated with gravity wave motions. These arise when the vertical wave number $m$ becomes small so that $1 / 2 H$ is a nonnegligible factor. With $H \sim 7 \mathrm{~km}$ in the middle atmosphere, this term becomes significant for waves with vertical wavelengths of $\sim 30 \mathrm{~km}$ or longer, where the change in background density becomes significant over the vertical wavelength of the wave. This compressibility effect is important in the interpretation of airglow images of gravity wave perturbations near the mesopause [e.g., Swenson et al., 2000]. As the vertical wavelength grows very large and $m \rightarrow 0$, the wave will undergo total internal reflection where the vertical group velocity can change sign (called a turning level). Above this level, the wave is evanscent. If turning levels occur both above and below, the wave is said to be ducted. Observational and modeling studies associated with these effects will be described further in section 3.2.2.

[14] Neglecting rotation effects but retaining the scale-height compressibility term and setting $m=0$ in equation (23) yields an equation describing the maximum horizontal intrinsic phase speed $\hat{c}_{\text {max }}$ prior to total internal reflection as a function of horizontal wavelength:

$$
\left|\hat{c}_{\max }\right|=N\left(k^{2}+l^{2}+\frac{1}{4 H^{2}}\right)^{-1 / 2}
$$

This gives a useful approximation to the fully compressible solution [Marks and Eckermann, 1995]. Figure 1 compares solutions for $\hat{c}_{\max }$ and the corresponding maximum intrinsic frequency $\hat{\omega}_{\max }$ versus horizontal wavelength for the fully compressible, incompressible, and approximate equation (29) solutions.

[15] Figure 1a shows that short horizontal wavelengths are much more easily reflected and will likely remain trapped at lower altitudes. Thus only waves with
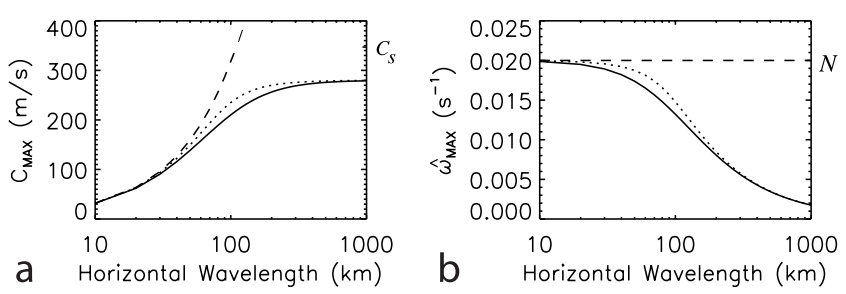

Figure 1. Analytical solutions derived from linear theory for the (a) maximum intrinsic phase speed ( $\left.\mathrm{c}_{\mathrm{MAX}}\right)$ and (b) maximum intrinsic frequency $\left(\omega_{\mathrm{MAX}}\right)$ at the point of total internal reflection, also known as the turning level. The dashed and solid lines are the incompressible and fully compressible solutions, respectively. The dotted line shows the approximation given by equation (29).

horizontal wavelengths $>10 \mathrm{~km}$ are generally considered important to middle-atmosphere dynamics. Although the vertical wavelength theoretically goes to infinity at the turning level (and therefore the WKB approximation should be violated), the theory embodied in equation (29) appears to provide a good approximation even for waves observed near this theoretical limit [Swenson et al., 2000].

[16] For high-frequency waves for which the Coriolis force can be neglected and for which $m^{2} \gg 1 / 4 H^{2}$, the dispersion relation simplifies to

$$
\hat{\omega}^{2}=\frac{N^{2}\left(k^{2}+l^{2}\right)}{k^{2}+l^{2}+m^{2}}=N^{2} \cos ^{2} \alpha,
$$

where $\alpha$ is the angle between lines of constant phase and the vertical. These are simple plane waves with zero velocity perturbations perpendicular to the plane of propagation. Group velocity simplifies to

$\left(c_{g h}, c_{g z}\right)=\left(\bar{u}_{h}, 0\right)+\frac{N m}{k_{h}^{2}+m^{2}} \frac{\left(m,-k_{h}\right)}{\left(k_{h}^{2}+m^{2}\right)^{1 / 2}}$,

where $c_{g h}$ is the horizontal component of group velocity, $\bar{u}_{h}$ the horizontal wind in the direction of propagation, and $k_{h}=\sqrt{k^{2}+l^{2}}$ is the horizontal wave number. Note that phase propagation is downward ( $m$ $<0$ ) for upward group velocity. In this form it can be seen that group velocity is parallel to lines of constant phase and perpendicular to phase propagation.

[17] High intrinsic frequency waves are observed not only in mesopause airglow image data [Swenson and Espy, 1995; Taylor and Garcia, 1995; Gardner et al., 1996; Taylor et al., 1997; Isler et al., 1997; Hickey et al., 1998; Haque and Swenson, 1999] but also in sublimb viewing infrared and microwave satellite observations in the stratosphere [Wu and Waters, 1996a, 1996b; Alexander, 1998; Dewan et al., 1998; Picard et al., 1998; McLandress et al., 2000] and in middle-atmosphere radar data [Reid et al., 1988; Fritts et al., 1990a, 1992; Fritts and Wang, 1991; Hoppe and Fritts, 1995; Mitchell and Howells, 1998]. 


\subsubsection{Medium-Frequency Waves: $N \gg \hat{\omega} \gg f$}

[18] For gravity waves with midrange intrinsic frequencies, very simplified relationships emerge that lend valuable insight into gravity wave properties and the effects due to changes in the background wind and stability. Letting $c_{h}$ represent the horizontal phase speed and $k_{h}=\sqrt{k^{2}+l^{2}}$, the dispersion relation then simplifies to

$$
\hat{\omega}=N\left|\frac{k_{h}}{m}\right|,
$$

and the vertical wave number can now be very simply related to the background wind and stability:

$$
|m|=\frac{N}{\left|\hat{c}_{h}\right|} .
$$

Note that $k_{h}>0$ for $\hat{c}_{h}=\left(c_{h}-\bar{u}_{h}\right)>0$, and $k_{h}<0$ for $\hat{c}_{h}<0$. Equations (32) and (33) demonstrate that vertical wavelength and intrinsic frequency are both proportional to the intrinsic phase speed $\hat{c}_{h}=c_{h}-\bar{u}_{h}$. The level where $c_{h}=\bar{u}_{h}(z)$ is a critical level for the wave where vertical wavelength shrinks to zero. This theoretical limit is never achieved in the real atmosphere because a host of instability and dissipation mechanisms become more likely as a wave approaches a critical level.

[19] The group velocity for medium-frequency gravity waves becomes

$$
\left(c_{g h}, c_{g z}\right)=\left(\bar{u}_{h}, 0\right)+\left(\frac{\hat{\omega}}{k_{h}}, \frac{-\hat{\omega}}{m}\right)
$$

and has the same magnitude as the phase propagation but opposite sign in the vertical. The time-dependent ray equations [Lighthill, 1978] show that a wave packet requires an infinite time to reach a critical level (with $m \rightarrow \infty$ ), implying that time-dependent and nonlinear effects will become important as a critical level is approached. Note, however, that wave transience and time dependence of the background flow can allow different interactions near the critical level. Departures from linear theory due to transience and nonlinearity are discussed in section 6 .

[20] The polarization relations under the mediumfrequency approximation take simple forms:

$$
\begin{gathered}
\tilde{p}=c_{g z} \widetilde{w}, \\
\widetilde{w}=-\frac{k_{h}}{m} \tilde{u}_{h}=\frac{k_{h} \hat{c}_{h}}{N} \tilde{u}_{h}=\frac{\hat{\omega}}{N} \tilde{u}_{h} .
\end{gathered}
$$

Note that group velocity, vertical wavelength, and intrinsic frequency increase as a wave propagates "upshear," defined as an altitude region where the intrinsic phase speed $\left|\hat{c}_{h}\right|$ is increasing. These simplified forms demonstrate that vertical velocity will grow proportionately larger than the horizontal velocity perturbations in these conditions, and pressure perturbations become increas- ingly important. The opposite is true for waves propagating "downshear" such as those approaching a critical level $\left(\left|\hat{c}_{h}\right| \rightarrow 0\right)$. In fact, it is the tendency for $\tilde{u} /\left|\hat{c}_{h}\right|$ to increase as the wave approaches a critical level that drives the tendency for instability and turbulence generation. These approximations for waves with midrange frequencies are inappropriate for most detailed analyses, but the set of very simple relationships that results provides an extremely valuable intuitive view of gravity wave properties and their relationship to background winds and stability.

\subsubsection{Low-Frequency Waves: $\hat{\boldsymbol{\omega}} \sim \boldsymbol{f}$}

[21] Low-frequency gravity waves, or inertia-gravity waves, are those for which the rotation of the Earth has an important influence. These wave perturbations have an interesting three-dimensional helical structure. Velocity perturbations in the direction perpendicular to the direction of propagation are no longer zero but grow proportionately larger as intrinsic frequency decreases toward $f$. For a zonally propagating wave the meridional velocity amplitude $\tilde{v}$ is

$$
\tilde{v}=-i \frac{f}{\hat{\omega}} \tilde{u} .
$$

An approximation to the dispersion relation appropriate for both low- and medium-frequency gravity waves is

$$
\hat{\omega}^{2}=N^{2} \frac{k_{h}^{2}}{m^{2}}+f^{2} .
$$

Any wave nearing a critical level (where the background wind speed approaches the horizontal phase speed and $m \rightarrow \infty$ ) will move toward lower intrinsic frequency where rotation effects become important. The true critical level is therefore where $\hat{\omega} \rightarrow f$, somewhere below the critical level described for mediumfrequency waves $\left(c_{h}=\bar{u}_{h}\right)$.

[22] Because the ratio of vertical to horizontal group velocity,

$$
\left|\frac{c_{g z}}{c_{g h}}\right|=\left|\frac{k_{h}}{m}\right|=\frac{\left(\hat{\omega}^{2}-f^{2}\right)^{1 / 2}}{N},
$$

becomes quite small for inertia-gravity waves relative to their higher-frequency counterparts, they can be found at large horizontal distances from their sources [Dunkerton, 1984; Eckermann, 1992]. They are very commonly seen in lower stratosphere observations.

[23] A number of observational techniques are sensitive to inertia-gravity waves. Balloon-borne measurements of horizontal wind profiles in the lower stratosphere enable inertia-gravity wave hodograph and rotary spectral analyses [Kitamura and Hirota, 1989; Barat and Cot, 1992; Tsuda et al., 1994a; Ogino et al., 1995, Hamilton and Vincent, 1995; de la Torre et al., 1996; Sato et al., 1994; Sato and Dunkerton, 1997; Shimizu and Tsuda, 
1997; Vincent and Alexander, 2000]. Limb viewing satellite measurements can also be sensitive to inertia-gravity waves [Fetzer and Gille, 1994, 1996; Eckermann and Preusse, 1999; Tsuda et al., 2000], and they are prominent signals in stratospheric radar and lidar observations [Sato, 1994; Mitchell et al., 1994; Sato et al., 1997; Riggin et al., 1997] and middle-atmosphere rocket sounding data [Hirota and Niki, 1985; Hamilton, 1991; Tsuda et al., 1992; Eckermann et al., 1995; Fritts et al., 1997a].

\subsection{Conservative Propagation and Departures}

[24] Gravity waves conserve wave pseudomomentum flux if they propagate without dissipation through a background atmosphere that varies only in the vertical. The vertical flux of horizontal pseudomomentum

$$
\left(F_{P x}, F_{P y}\right)=\bar{\rho} c_{g z} \frac{E}{\hat{\omega}}(k, l)
$$

is related to the Reynolds stress and vertical flux of horizontal momentum under these assumptions as

$$
\left(F_{P x}, F_{P_{y}}\right)=\bar{\rho}\left(1-f^{2} / \hat{\omega}^{2}\right)\left(\overline{u^{\prime} w^{\prime}}, \overline{v^{\prime} w^{\prime}}\right) .
$$

Dissipation results from processes such as radiative damping [Fels, 1984; Zhu, 1994], wave-wave and wavemean flow interactions [Broutman and Grimshaw, 1988; Sutherland, 2000, 2001], and wave breaking and instability processes (see section 6). We note here only that nonlinearity and time dependence usually prevent the attainment of amplitudes anticipated by linear, inviscid, steady wave theory.

[25] Viscosity and molecular diffusion are generally not important dissipation mechanisms except in the thermosphere where the exponentially decreasing density and growth of kinematic viscosity with altitude ultimately allow these to dominate over other dissipation processes. (We note here that the "viscosity waves" proposed by Hocking et al. [1991] are, in reality, the viscous solutions of the Orr-Sommerfeld equation [e.g., Fritts and Geller, 1976]. They do not propagate, and they do not have a dispersion relation. Thus their role is to eliminate, not create, the artificial singularities that occur in the linear inviscid equations, and they cannot play the role proposed by Hocking et al. [1991] accounting for enhanced radar backscatter.) This growth of kinematic viscosity with altitude accounts for the turbopause at an altitude typically $\sim 110 \mathrm{~km}$.

[26] The negative of the zonal mean pseudomomentum flux is the gravity wave contribution to the more general Eliassen-Palm flux (EP flux) that is commonly used to describe planetary-scale wave effects [Edmon et al., 1980; Miyahara et al., 1986]. The divergence of the EP flux is proportional to the force exerted on the mean flow. In the case of gravity waves, the zonal average is replaced by an average over some smaller, but still large, area such as a GCM grid scale, and the force on the background wind on that scale becomes

$$
(\bar{X}, \bar{Y})=-\frac{\varepsilon}{\bar{\rho}} \frac{\partial}{\partial z}\left(F_{P_{x}}, F_{P_{y}}\right) .
$$

This is the wave driving (or wave-induced force) [Andrews and McIntyre, 1976] that would be used for studying wind evolution in the momentum conservation equation for the background flow as in equations (1) and (2). The force is a vector quantity such that the direction and sign of the force are always the direction and sign of the horizontal phase propagation in the frame of reference moving with the wind (i.e., the horizontal intrinsic phase speed of the wave).

[27] The factor $\varepsilon$ has been called an efficiency [Holton, 1982] or intermittency [Alexander and Dunkerton, 1999] factor, and it can be thought of as describing the fractional coverage of the wave dissipation event within the larger-scale space- and/or time-averaging interval. The intermittency factor is a necessary ingredient in gravity wave parameterization estimates of $(\bar{X}, \bar{Y})$ when the input parameters are describing mean gravity wave properties. We note, however, that for observational estimates of $\left(F_{P x}, F_{P y}\right)$ and $(\bar{X}, \bar{Y})$, such as derived from time-averaged radar data, the $\varepsilon$ factor is already included.

[28] Gravity waves also have an associated energy flux, so departures from conservative propagation will lead to energy dissipation and local heating of the atmosphere. If wave dissipation involves turbulence generation, then mixing and turbulent heat, momentum, and constituent transport will also occur. Direct heating has been neglected in most GCMs that parameterize gravity wave effects. Models of the middle atmosphere, however, generally require parameterized vertical mixing that is commonly called eddy diffusion. Such mixing could, for example, result from convective overturning of material surfaces and subsequent turbulence following gravity wave breaking (see section 6.2.1).

[29] A momentum eddy diffusion coefficient associated with gravity wave dissipation may be approximated for a single wave [Lindzen, 1981; Holton, 1982] as

$$
D_{M}=\frac{\hat{c}|(\bar{X}, \bar{Y})|}{N^{2}} .
$$

This coefficient is related to the thermal eddy diffusion coefficient via the Prandtl number. The value of the Prandtl number associated with gravity wave breaking is poorly defined at present and may vary with height. It is expected to be large and highly variable based on numerical and theoretical models of gravity wave convective instability [Chao and Schoeberl, 1984; Fritts and Dunkerton, 1985; Coy and Fritts, 1988; McIntyre, 1989]. Assessments of turbulent thermal diffusion required to account for observed atmospheric structure in global models likewise imply large turbulent Prandtl numbers and smaller thermal diffusion coefficient than the momentum diffusion coefficient predicted by equation (43) [Strobel et al., 1985, 1987; Holton and Schoeberl, 1988; Smith and Brasseur, 1991; Huang and Smith, 1991]. 
While equation (43) with an arbitrary scaling factor is very commonly applied, this relation remains to be confirmed and extended to multiple waves by direct numerical simulation.

[30] Departures from linear theory also occur because of the time dependence of the background flow and the spatial and temporal variations in wave activity (i.e., wave packets). In the case of a gravity wave packet that is localized in space and/or time, the result is a spread of horizontal and/or vertical wave numbers and a corresponding spread of intrinsic frequencies. A gravity wave packet in a shear flow may achieve large amplitudes, but it never attains a singular critical level solution. Spatial localization of a wave packet is also now understood to have some surprising effects. Gravity waves due to local topography comprise a continuous spectrum of wave numbers and propagation directions. When such a spectrum of gravity waves is incident on a veering wind, the result is a monotonic decrease in momentum flux and a turning of the direction of wave drag with altitude as successive portions of the spectrum are removed by critical levels [Hines, 1988a; Shutts, 1995, 1998; Broad, 1995, 1999]. At larger wave amplitudes, localization of a wave packet in the vertical can result in its penetration via nonlinear "self-acceleration" beyond its linear critical level [Fritts and Dunkerton, 1984], while localization horizontally and vertically can enable penetration through a turning level [Sutherland, 2000] (see section 6.2.3).

[31] Time dependence of the flow in which a gravity wave propagates also influences wave characteristics, enabling the formation of caustics, inducing wave number transport, and either accelerating or retarding the approach toward unstable local wave amplitudes [Broutman and Young, 1986; Bruhwiler and Kaper, 1995; Eckermann, 1997; Buckley et al., 1999; Walterscheid, 2000]. Given the importance of (or lack of) these processes in the spectral evolution of gravity waves in the middle atmosphere and the current debate within the community over these issues, this topic will be reviewed more fully in section 6 .

\section{SOURCES AND PROPAGATION}

\subsection{Gravity Wave Sources}

[32] A complete survey of the gravity wave sources important for the middle atmosphere is beyond the scope of this paper. Indeed, such a survey is beyond our current state of knowledge based on the extensive theoretical, modeling, and observational efforts to date. Instead, the purpose of this section is to identify the dominant sources, their spatial and temporal variability, and the general characteristics of gravity waves arising from them as we understand them at present. The most obvious sources include topography, convection, and wind shear, although other sources may also be significant at preferred sites or in association with specific larger-scale dynamics. Examples of other sources that are likely to be important statistically include adjustment of unbalanced flows in the vicinity of jet streams and frontal systems, body forcing accompanying localized wave dissipation, and wave-wave interactions. Less significant sources which may, nevertheless, be important at higher altitudes include auroral heating and eclipse cooling.

\subsubsection{Topographic Generation}

[33] Mountain waves have been studied extensively over the past three decades using theoretical, numerical, and observational methods. As a result, idealized twodimensional (2-D) responses to 2-D topography are reasonably well understood in terms of nonlinear wave dynamics and hydraulic theory [Long, 1955; Smith, 1985; Durran and Klemp, 1987; Nance and Durran, 1998; Lott, 1998; Farmer and Armi, 1999]. Responses to three-dimensional (3-D) topography have also been quantified increasingly in a number of measurement programs and numerical studies, yielding estimates of mountain wave scales, amplitudes, and momentum fluxes. Horizontal wavelengths for vertically propagating waves are typically tens to hundreds of kilometers, while amplitudes vary from small to breaking [Lilly and Kennedy, 1973; Nastrom and Fritts, 1992; Chan et al., 1993; Dörnbrack et al., 1999; Leutbecher and Volkert, 2000]. Horizontal and vertical cross sections of a wave structure anticipated over northern Scandinavia in a 3-D mesoscale simulation by Dörnbrack et al. [1999] are shown in Figure 2 and exhibit the large-scale thermal structure (areas of low temperature in Figure 2) believed to trigger polar stratospheric clouds (PSC) and ozone depletion. Such simulations, however, do not yet capture the responses often observed over smaller-scale topography [Nastrom and Fritts, 1992].

[34] Momentum fluxes likewise vary greatly but are typically in the range of $\sim 0.1$ to a few $\mathrm{m}^{2} \mathrm{~s}^{-2}$ (per unit mass, or $\sim 0.01-0.5 \mathrm{~Pa}$ ) in the upper troposphere and lower stratosphere based on in situ aircraft and radar measurements and large-scale cross-mountain pressure distributions [Lilly and Kennedy, 1973; Lilly and Lester, 1974; Smith, 1978; Kennedy and Shapiro, 1979; Shutts et al., 1988; Fritts et al., 1990b; Sato, 1994]. Momentum fluxes are also preferentially associated with the smaller horizontal scale waves that are vertically propagating because of their larger vertical velocities. Maximum (generally negative in Figure 2) momentum fluxes were inferred at horizontal wavelengths between $\sim 10$ and 100 km by Nastrom and Fritts [1992] (see Figure 3).

[35] The Global Atmospheric Sampling Program (GASP) and more recent aircraft observations also enabled assessments of the statistical importance of mountain waves over rough and smooth terrain and relative to other significant sources. These showed horizontal velocity and temperature variances to be $\sim 2-3$ times higher over significant topography compared to plains and oceans, independent of other sources [Nastrom et 

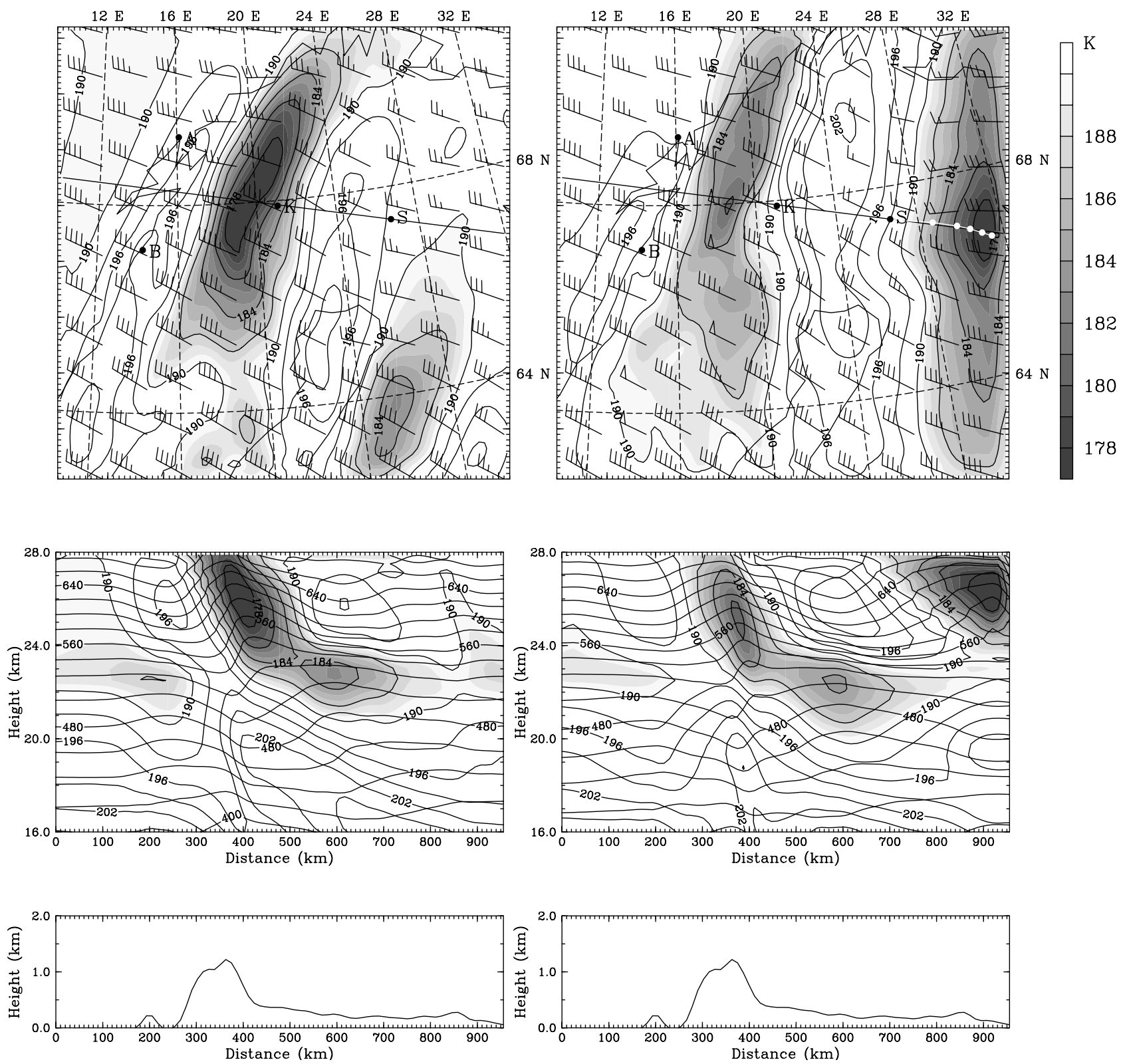

Figure 2. (top) Horizontal and (middle) vertical cross sections of temperature and horizontal wind in a 3-D mesoscale simulation of flow over northern Scandinavia. (bottom) Terrain height beneath the vertical cross section. Modified after Dörnbrack et al. [1999].

al., 1987; Jasperson et al., 1990; Bacmeister et al., 1990a] and $\sim 5$ times higher than regions having no obvious meteorological sources [Fritts and Nastrom, 1992]. Because of the importance of such waves for mean circulations a number of authors have attempted to parameterize their specific effects in large-scale models (see section 7). The importance of mountain waves relative to other significant sources is discussed further in section 5 .

[36] Of relevance to the middle atmosphere, then, mountain waves have dominant scales (in terms of momentum fluxes) of $\sim 10-100 \mathrm{~km}$, phase speeds near zero and vertical wavelengths dictated by the local static stability and mean wind in the plane of wave propagation [Bacmeister et al., 1990b]. Indeed, Bacmeister [1993] has argued that mountain waves likely account for a large fraction of zonally averaged wave-induced force (wave drag) in the mesosphere, although this result is based on predictions of Lindzen-type parameterizations (see section 7) and remains to be demonstrated observationally.

\subsubsection{Convective Generation}

[37] Although it has been known for decades that convection can excite gravity waves, it has only been in more recent years that observations and models have begun to characterize the waves generated by this source and the mechanisms within convection responsible for their generation. In fact, there is at the time of this 

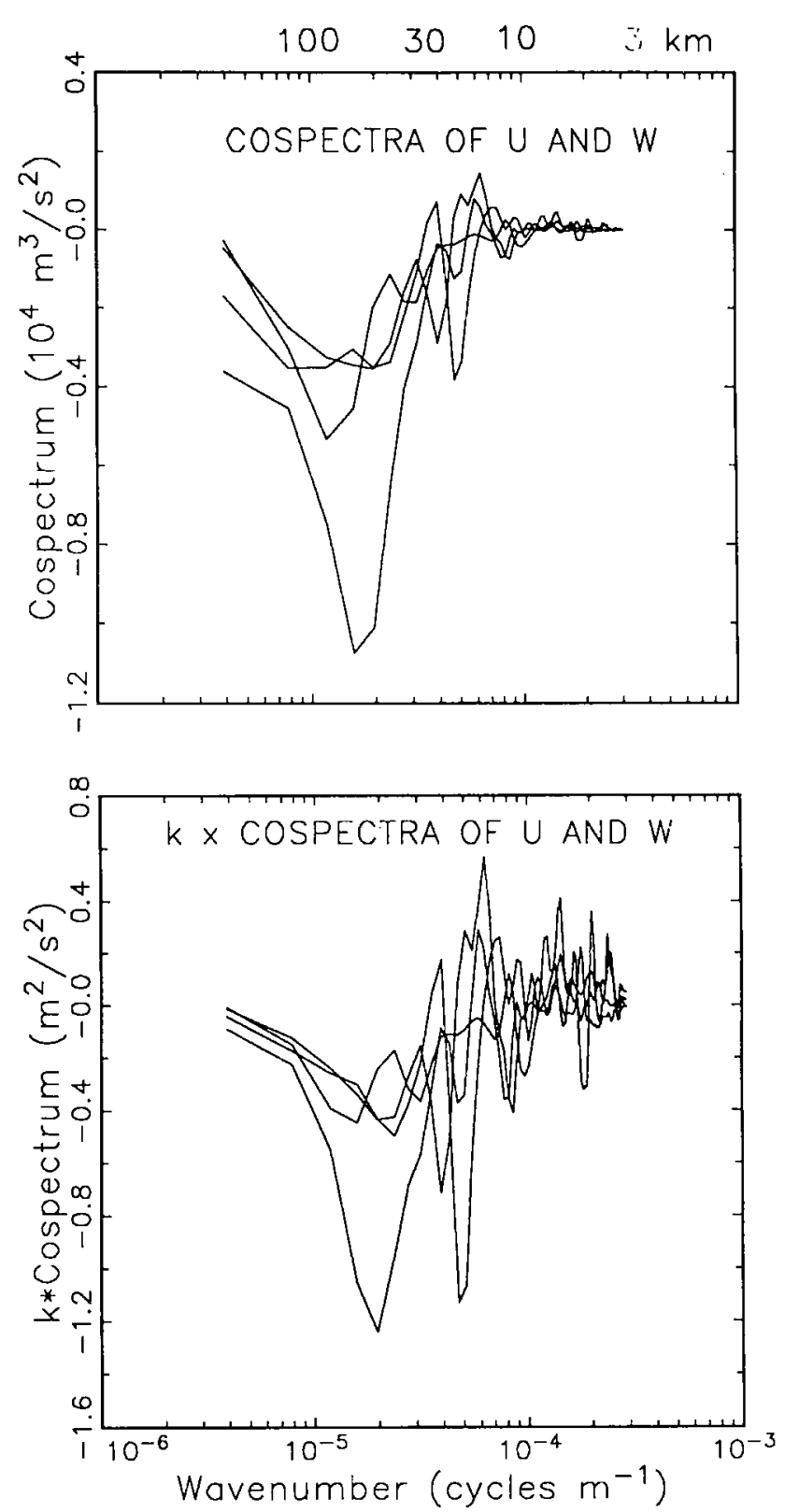

Figure 3. Spectra of momentum flux in (top) standard and (bottom) variance content forms computed for four flights over the central Rockies. Note that the dominant fluxes in each case are largely at horizontal scales between 10 and $100 \mathrm{~km}$. After Nastrom and Fritts [1992] (reprinted with permission of the American Meteorological Society).

review still considerable controversy and ongoing research aimed at understanding this wave generation mechanism.

[38] One of the difficulties in characterizing this source is its inherent intermittency. Observations of high-frequency waves in the stratosphere have shown a close correspondence with deep convective clouds (see Figure 4) [Sato, 1992, 1993; Alexander and Pfister, 1995; Sato et al., 1995; Dewan et al., 1998; McLandress et al., 2000; Alexander et al., 2000]. Observational estimates of momentum flux are fewer but show significant variabil- ity. Local magnitudes range up to $\sim 0.03-0.2 \mathrm{~Pa}$ [Sato, 1993; Alexander and Pfister, 1995; Alexander et al., 2000], and longer-term averages peak at $\sim 1-5 \times 10^{-3} \mathrm{~Pa}$ [Sato and Dunkerton, 1997; Sato et al., 1997; Vincent and Alexander, 2000].

[39] Waves generated by convection are not characterized by a single prominent phase speed or frequency as is the case for topographic waves. Instead, convection can generate waves throughout the full range of phase speeds, wave frequencies, and vertical and horizontal scales. The low-frequency waves, in particular, may be observed in the middle atmosphere at large horizontal distances from the convective source, making correlation with clouds or other indicators of convection more difficult. In the tropics, however, far from topography and regions of baroclinic instability, the occurrence of inertia-gravity waves has been linked to convection as the source [Pfister et al., 1986; Tsuda et al., 1994a; Karoly et al., 1996; Shimizu and Tsuda, 1997; Wada et al., 1999; Vincent and Alexander, 2000].

[40] Model studies of waves generated by convection (Figure 5) have greatly enhanced our understanding of these phenomena. Convection involves a time-varying thermal forcing associated with latent heat release that can interact with overlying stable layers and shear in complex ways that are not fully understood. Three simplified mechanisms have been proposed to describe convective generation: (1) pure thermal forcing, (2) an "obstacle" or "transient mountain" effect, and (3) a "mechanical oscillator" effect.

[41] For pure thermal forcing in the absence of strong shear the heating depth and the vertical wavelength of the gravity waves generated are surprisingly approximately equal [Alexander et al., 1995; Piani et al., 2000]. This relationship (demonstrated by Salby and Garcia [1987] for larger-scale waves) arises because the heating projects most strongly onto a wave with vertical wavelength twice the depth of the heating, but the wave is then refracted to half that vertical wavelength as it propagates across the twofold increase in buoyancy frequency at the tropopause (see equation (33)). Bergman and Salby [1994] used global cloud imagery at tropical latitudes to estimate the planetary-scale and long-wavelength, low-frequency gravity wave responses with this model, and the far-field effects of the waves generated have been extended through the mesosphere by Garcia and Sassi [1999]. The linear mechanism used in these studies has been validated for tropical planetary-scale waves, but the amplitudes are generally larger than those in corresponding nonlinear models [Manzini and Hamilton, 1993]. The gravity wave momentum fluxes derived from these cloud data sets are still highly uncertain. Pandya and Alexander [1999] further describe how the local mesoscale environment surrounding the storm can modify the smaller-scale gravity wave response in the stratosphere from a latent heat source. Observational evidence for the thermal forcing mechanism of gravity wave generation is given by McLandress et al. [2000] 


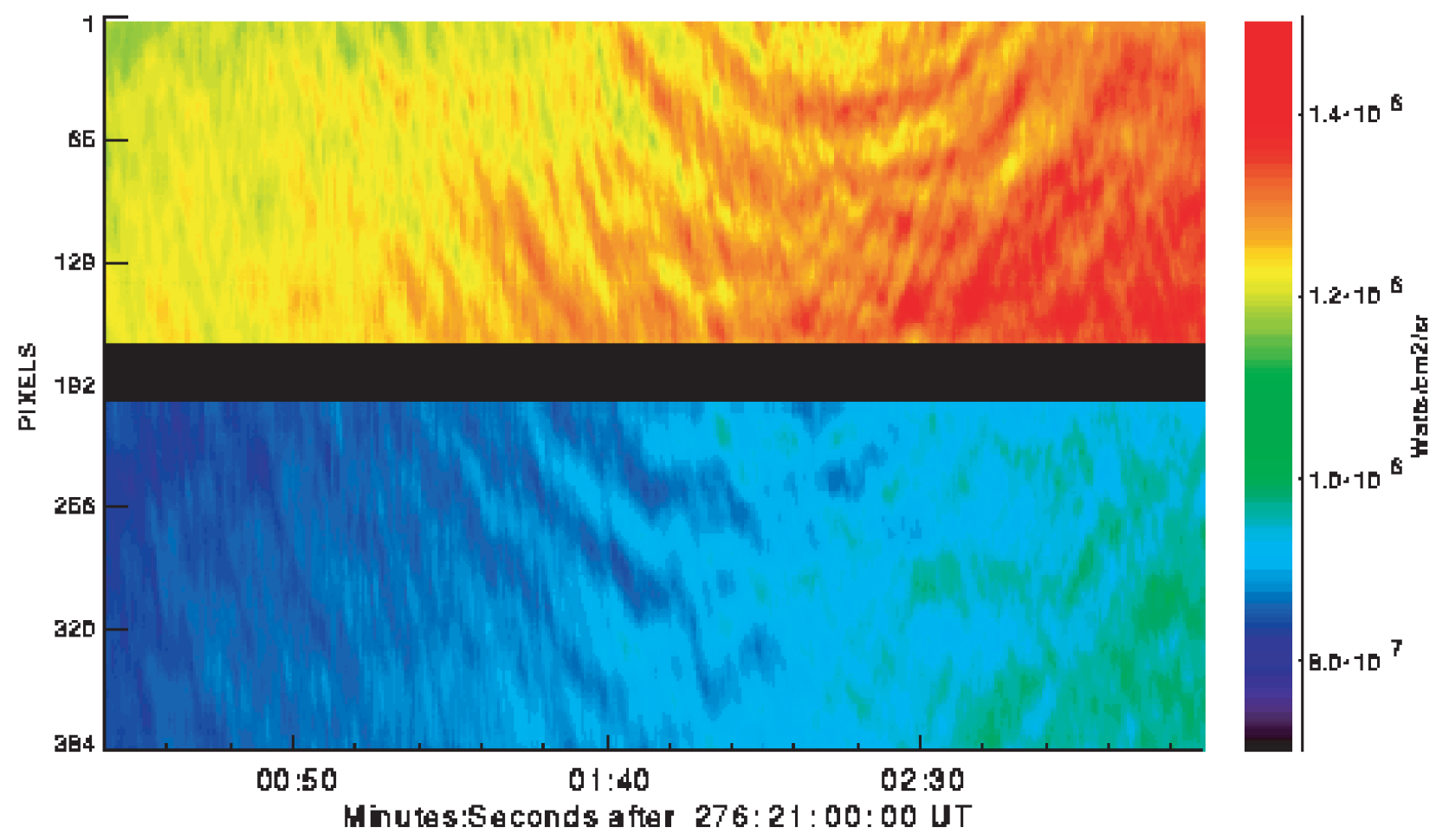

Figure 4. Images of infrared emissions from the stratosphere observed by the SPIRIT III radiometer from the MSX satellite. Circular phase fronts of waves with horizontal wavelength $\sim 25 \mathrm{~km}$ can be seen in these images that were centered around a deep convective cloud below in the troposphere. After Dewan et al. [1998, Figure 1].

(Figure 6), although its importance relative to other mechanisms could not be quantified. Pure thermal forcing would be expected to generate an isotropic wave field, with anisotropies occurring primarily via background wind filtering effects.

[42] The obstacle effect was descibed by Clark et al. [1986] for short horizontal wavelength waves in the troposphere generated by boundary layer convection [Kuettner et al., 1987], but the ideas developed in their study have also contributed to understanding the larger horizontal scale waves in the middle atmosphere generated by convection. The obstacle effect can be thought of as analogous to topographic wave generation where the convective heating modifies the shape of isentropes at the bottom of a stable layer which is moving relative to the convective obstacle. Clark et al. [1986] found that both the pure thermal forcing and the obstacle effect produced gravity waves in the overlying stable layer but that the obstacle effect generated much larger amplitude waves. Pfister et al. [1993a, 1993b] explicitly modeled a linear version of this mechanism and found evidence for it in stratospheric aircraft observations over convection. Pfister et al. [1993b] also described how the time dependence of the obstacle (or transient mountain) affects the phase speed spectrum of the waves that are generated. Observational evidence for the obstacle effect has appeared in radiosonde analyses of low-frequency waves [Vincent and Alexander, 2000; Alexander and Vincent,
2000]. This mechanism is associated with an anisotropic wave spectrum with a preference for generating waves propagating opposite to the mean wind relative to the obstacle.

[43] Fovell et al. [1992] described the "mechanical oscillator" mechanism as a periodic and localized momentum source term that generates waves with a frequency equal to the oscillation frequency. This mechanism is not distinct from the time-varying thermal source when that source oscillates with a regular period [Clark et al., 1986; Alexander et al., 1995; Pandya and Alexander, 1999]. Lane et al. [2001] describe a similar mechanism acting in their 3-D model that had little or no shear in the forcing region, with some added constraints. In their conceptual model the parcel oscillates about its level of neutral buoyancy at the local tropospheric buoyancy frequency which limits this mechanism to generating only very high frequency gravity waves in the upper troposphere at altitudes above any lower minima that occur in the $N$ profile. For the mechanical oscillator mechanism, anisotropies in the wave spectrum can result from background wind effects in a manner similar to the obstacle effect [Fovell et al., 1992].

[44] In summary, the three simplified excitation mechanisms that have been proposed may all be important, depending strongly upon the local shear and the vertical profile and time dependence of the latent heating. In reality, the three mechanisms are not distinct but cou- 

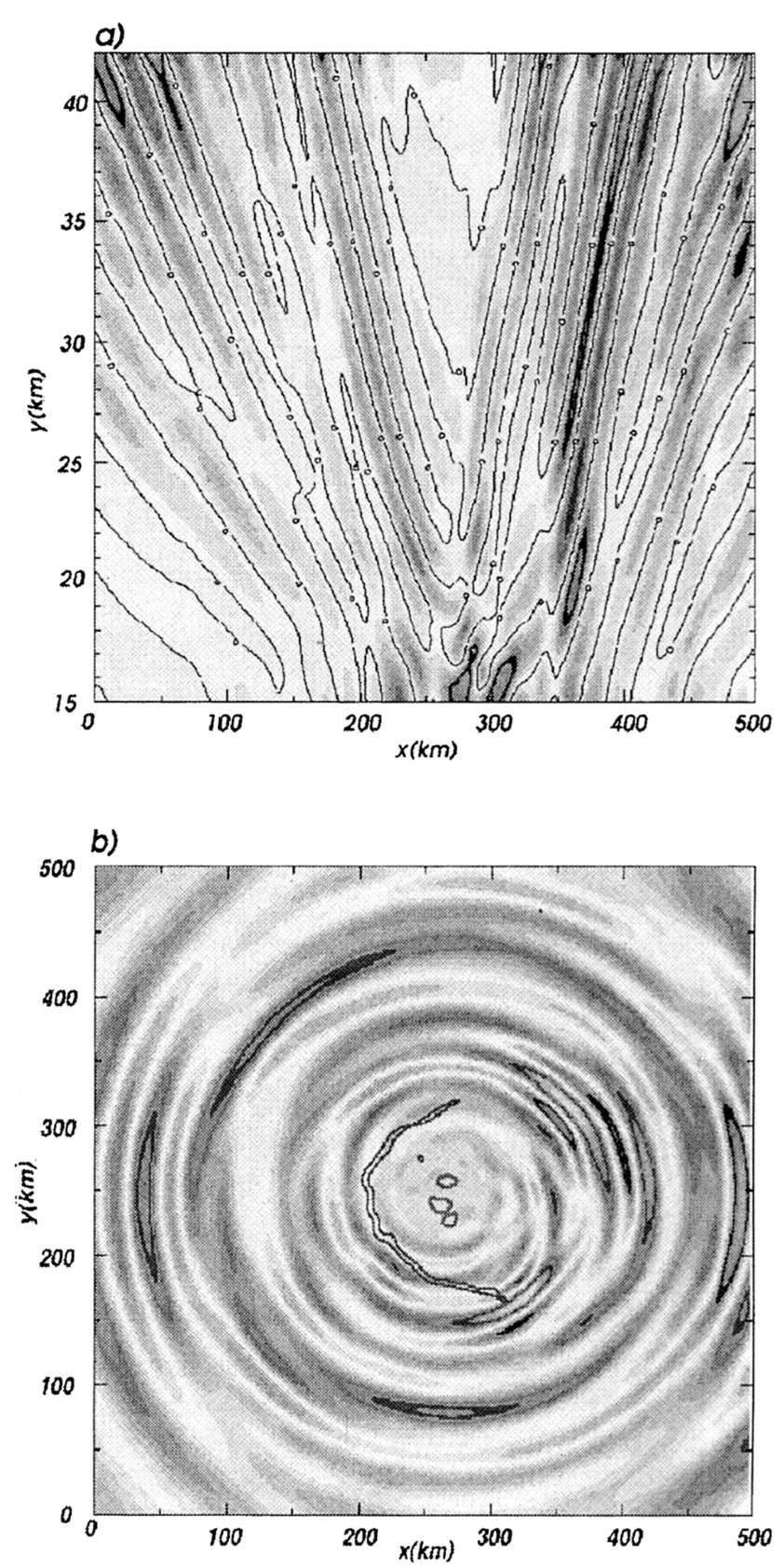

Figure 5. Three-dimensional study of gravity waves generated by convection in a mesoscale model with parameterized microphysics. (a) Vertical velocity patterns in a cross section in the vertical $(z)$ and zonal $(x)$ plane at $y=250 \mathrm{~km}$. (b) The $x-$ $y$ cross section of vertical velocity at $z=40 \mathrm{~km}$. Also shown in Figure $5 \mathrm{~b}$ are the surface gust front (arc-shaped solid line) and regions of strong latent heating in the troposphere (small solid contours). After Piani et al. [2000, Figure 4] (reprinted with permission of the American Meteorological Society.)

pled. However, one mechanism or another may serve to largely explain a set of observations with certain wind shear conditions or may serve to explain the generation of a certain class of waves, such as long vertical wavelength wave generation associated with deep convective heating.

\subsubsection{Shear Generation}

[45] The excitation of gravity waves by unstable shears has been studied for many years but remains one of the least quantified sources of gravity wave activity. A major challenge in these efforts was to account for the emergence of gravity waves that are propagating away from the shear layer on a timescale competitive with the most rapidly growing Kelvin-Helmholtz $(\mathrm{KH})$ instability. This proved difficult for linear modes of instability for which the growth rate depends on the horizontal wave number. The subharmonic interaction, or pairing, of $\mathrm{KH}$ modes was proposed by Davis and Peltier [1979] to provide such excitation, but Fritts [1984b] showed pairing not to be an efficient mechanism when the exterior flow is stably stratified. By invoking a nonlinear interaction between KH and propagating modes, however, Fritts [1984b] and Chimonas and Grant [1984] were able to account for rapid excitation of propagating gravity waves. This "envelope" radiation is essentially excitation of a gravity wave by the packet-scale motions accompanying a packet of coherent $\mathrm{KH}$ billows evolving together in an unstable shear layer of finite horizontal extent. Two aspects of this mechanism have been assessed more recently in greater detail. Bühler et al. [1999] and Bühler and McIntyre [1999] examined wave radiation from the collapse of a mixed region due to $\mathrm{KH}$ billows of finite extent and concluded that this source could not be neglected in the momentum budget at greater altitudes. The mechanism envisioned by Bühler et al. [1999] is represented schematically in Figure 7. Scinocca and Ford [2000] reexamined the nonlinear radiation from spatially localized KH billows and concluded, like Fritts [1984b], that envelope radiation is a viable mechanism for gravity wave generation and likely an important contributor to the mesospheric momentum budget.

[46] Assuming envelope radiation to be the predominant shear excitation mechanism, we can infer characteristic horizontal scales of tens to $\sim 100 \mathrm{KH}$ wavelengths or a few to tens of kilometers, with horizontal phase speeds comparable to the mean wind at the unstable shear layer. There is, nevertheless, some observational evidence suggesting that certain linear modes of shear instability can be excited [Mastrantonio et al., 1976; Lalas and Einaudi, 1976]. Under such circumstances the gravity wave scales are determined by the character of the shear flow and tend to be considerably larger than $\mathrm{KH}$ wavelengths.

\subsubsection{Geostrophic Adjustment}

[47] Most evidence for gravity wave excitation accompanying the restoration of balanced flow, commonly termed geostrophic adjustment, even though the balance need not be geostrophic, comes from theoretical or numerical treatments of adjustment processes. In such a process, an initial or evolving unbalanced flow relaxes to a new balanced state via both a redistribution of mean momentum, energy, and potential vorticity and a radiation of excess energy away as inertia-gravity waves. Such 
(a) $15 \mathrm{~N} \mathrm{JJA}$ (MLS variances at $38 \mathrm{~km}$ )

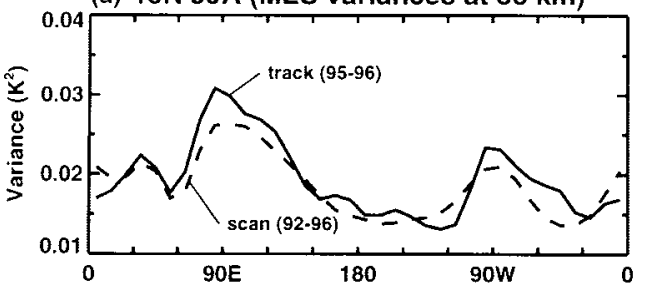

(c) $15 \mathrm{~N}$ JJA (Outgoing LW Radiation)

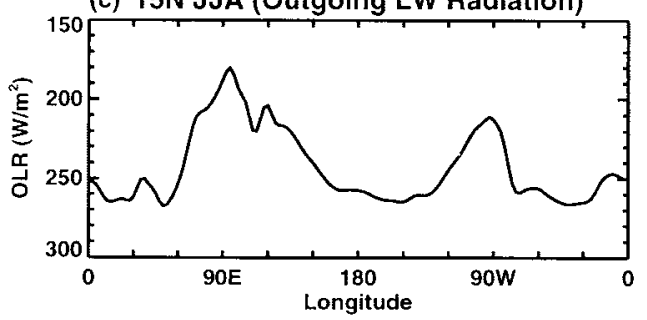

(b) 15S DJF (MLS variances at $38 \mathrm{~km}$ )

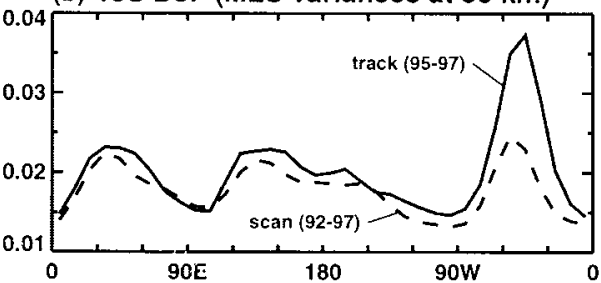

(d) 15S DJF (Outgoing LW Radiation)

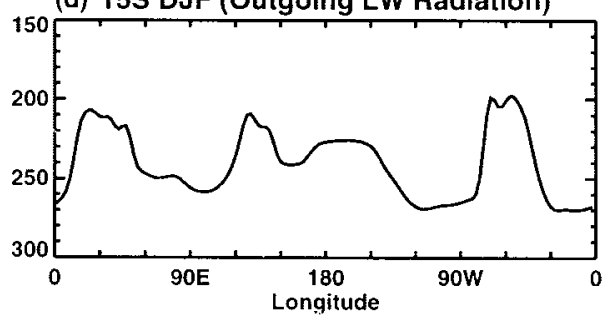

Figure 6. (a) Observations of gravity wave temperature variance as a function of longitude at $15^{\circ} \mathrm{N}$ from the Microwave Limb Sounder (MLS) instrument on the UARS satellite, averaged over the June-July-August season. (b) Observations of Outgoing Longwave Radiation for the same season and locations plotted on an inverse scale. The two plots show a close correspondence between gravity wave variance and infrared emission from deep, cold convective clouds. The gravity waves were also noted to have large horizontal phase speeds and very long vertical wavelengths. The close correspondence between Figures $6 \mathrm{a}$ and $6 \mathrm{~b}$ provided observational evidence that long vertical wavelength gravity waves are preferentially excited by deep latent heating. After McLandress et al. [2000, Figure 8].

unbalanced initial flows can arise, for example, where cross-stream pressure gradients are varying along the fluid motion (requiring an acceleration or deceleration of the motion), accompanying frontal evolution and baroclinic instability, or in response to local body forces due to gravity wave dissipation and momentum flux divergence. The first of these was modeled in 2-D and 3-D by Fritts and Luo [1992] and Luo and Fritts [1993] as adjustments to unbalanced initial conditions and was found to lead to both new balanced 2-D or 3-D flows and a spectrum of inertia-gravity waves that owe their character to the geometry of the source perturbation. Observations of inertia-gravity wave activity by Hirota and Niki [1985] and Thomas et al. [1992] exhibiting downward phase progression above the jet stream and upward phase progression below the jet stream appear to provide some support for this conceptual model. Further support comes from the more recent study by Guest et al. [2000] who found both a high correlation of inertia- gravity wave observations with a synoptic pattern having a jet stream nearby and a tendency for reverse ray tracing to suggest the jet stream as the wave source. Our understanding of such adjustment processes is nevertheless poor at this time and will benefit from further research efforts.

[48] Frontogenesis and baroclinic instability were found by O'Sullivan and Dunkerton [1995], Griffiths and Reeder [1996], and Reeder and Griffiths [1996] to yield similar results, with both adjusted mean and waves scales largely defined by the character of the unbalanced flow. An example of the inertia-gravity waves emitted accompanying adjustment of an evolving baroclinic flow is shown in Figure 8 [O'Sullivan and Dunkerton, 1995]. As discussed above, gravity waves are seen to arise preferentially in regions of strong flow deceleration.

[49] Gravity waves arising from local body forces due to dissipating gravity waves were considered theoretically by Zhu and Holton [1987] and more recently by

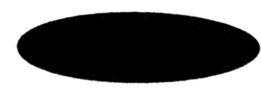

A

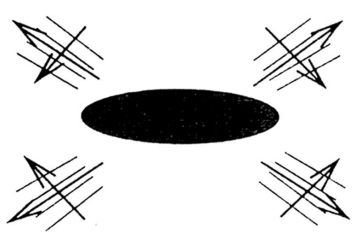

B

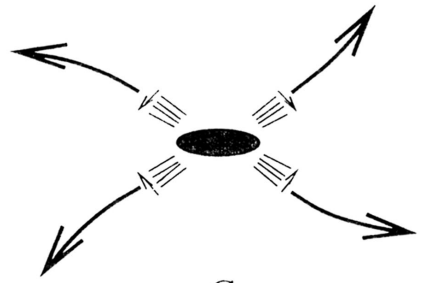

C

Figure 7. Schematic of the consequences of collapse of a train of $\mathrm{KH}$ billows which trigger radiation of gravity waves at larger scales. After Bühler et al. [1999] (reprinted with permission of the American Meteorological Society). 


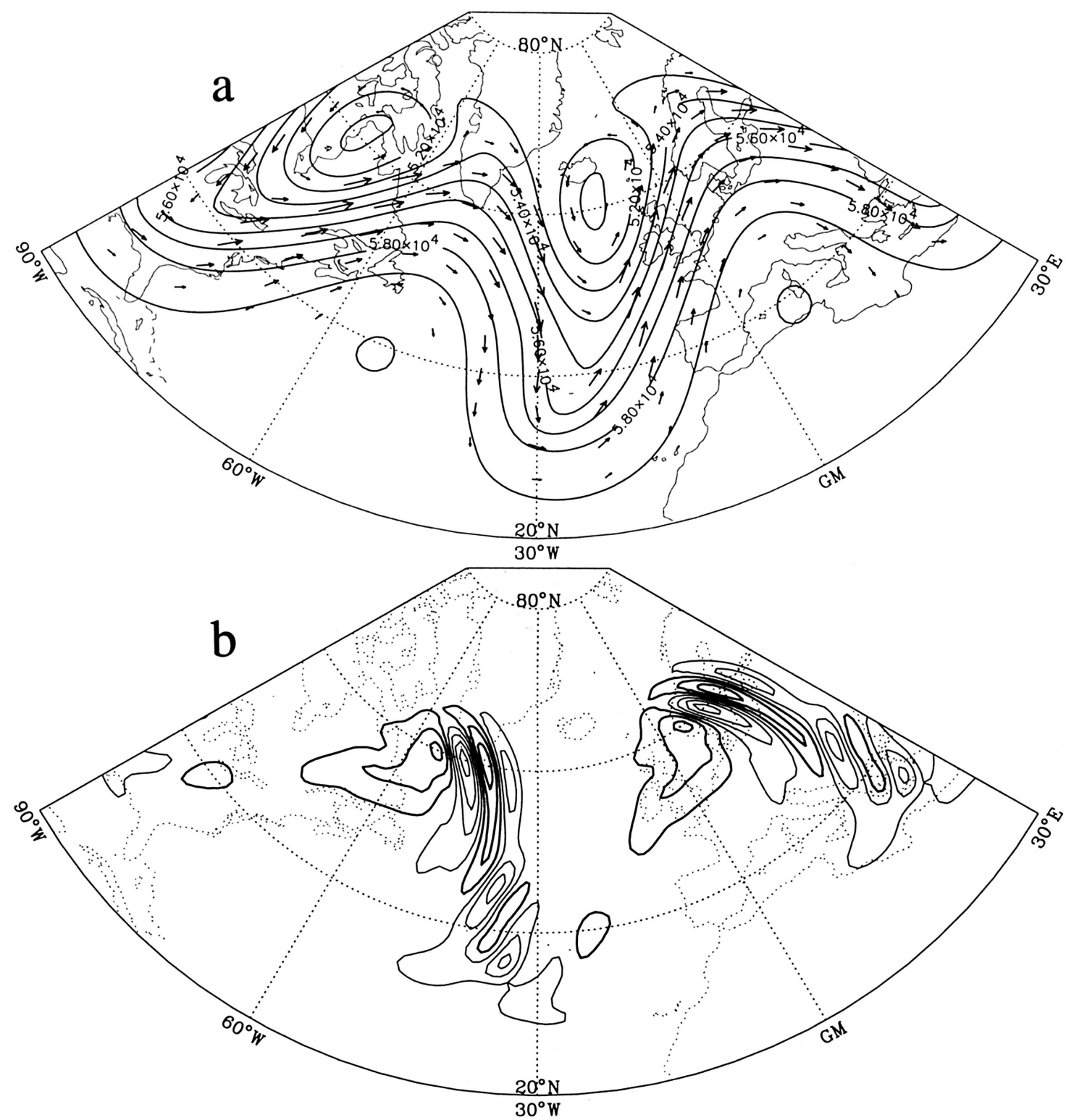

Figure 8. The (a) $500 \mathrm{hPA}$ geopotential height and winds and (b) $130 \mathrm{hPA}$ horizontal divergence (bottom) displaying the excitation of inertia-gravity waves by geostrophic adjustment processes. After O'Sullivan and Dunkerton [1995] (reprinted with permission the American Meteorological Society).

Vadas and Fritts [2001]. In all cases, the response is a combination of an adjusted mean (with redistributed momentum or potential vorticity), an altered mean thermal structure, and radiated inertia-gravity waves ensuring energy conservation in the absence of dissipation. The study by Vadas and Fritts [2001] considered a temporally evolving body force and showed (1) that the adjusted mean state depends on the momentum impulse and forcing geometry but not on the forcing duration and (2) that the radiated inertia-gravity waves are constrained by both the geometry and the temporal structure of the body forcing. Thus, while both long- and short-duration forcing (having the same geometry and momentum impulse) induce the same balanced mean flow, their gravity wave responses are quite different, with long-duration forcing strongly suppressing gravity waves having periods shorter than the forcing duration. The adjustment processes discussed here are also closely analogous to the radiation of gravity waves by a collapsing mixed layer due to shear instability examined by Bühler et al. [1999] and discussed above, though the source here is due to an addition, rather than a redistribution, of momentum.

[50] Scales of inertia-gravity waves arising from adjustment or body forcing processes are imposed by the source spatial scales and further constrained by the source timescales. Typical vertical scales are a few kilometers or more, with horizontal scales along the direction of propagation of $\sim 10-100$ times larger. Wave periods likely vary from an hour or so out to the inertial period. In cases where the source is elongated in one direction horizontally, the predominant direction of 
64-km variances
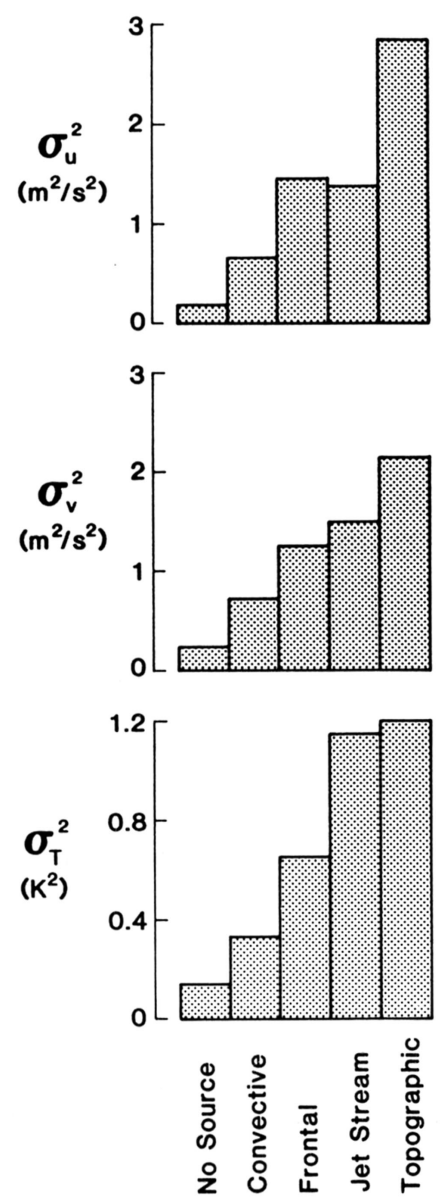

256-km variances
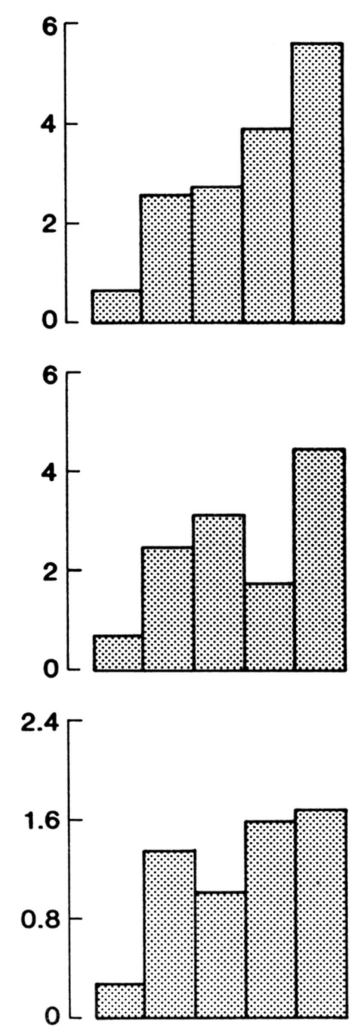

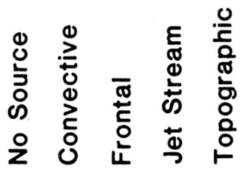

Figure 9. (top) Zonal velocity, (middle) meridional velocity, and (bottom) temperature variances in (left) 64-km and (right) $256-\mathrm{km}$ data segments associated with various gravity wave sources. After Fritts and Nastrom [1992] (reprinted with permission of the American Meteorological Society).

propagation of the excited inertia-gravity waves is normal to the source axis.

[51] A comparison of gravity wave velocity and temperature variances associated with various sources computed using GASP data near the tropopause is shown in Figure 9 [Fritts and Nastrom, 1992]. These variances, for 64- and 256-km flight segments, suggest that topography, jet stream shears, and convective and frontal activity all contribute substantially to gravity wave excitation where these sources are prevalent.

\subsubsection{Wave-Wave Interactions}

[52] Nonlinear wave-wave interactions are arguably one of the more important mechanisms in energy exchanges, amplitude constraints, and spectral evolution for gravity waves in the middle atmosphere. Their roles and importance are not universally acknowledged, however, and there remains much to be done in quantifying their dynamics and effects.

[53] As a source of gravity waves the atmospheric community has taken guidance from oceanographers and attributed energy transfers to resonant and nonresonant interactions of various forms. There has been a long debate over the efficiency of such interactions and the validity of the various approaches in the oceanographic and atmospheric communities. Yet laboratory experiments and direct numerical simulations demonstrate that specific interactions, e.g., the parametric subharmonic instability (or PSI, see section 6.1), can be efficient and robust under a variety of circumstances $[\mathrm{Mc}$ Ewan, 1971; McEwan and Robinson, 1975; Mied, 1976; Dunkerton, 1987; Thorpe, 1994; Vanneste, 1995; Staquet and Sommeria, 1996]. Indeed, Klostermeyer [1984, 1990] has argued for evidence of PSI in radar measurements of the middle atmosphere. Thus wave-wave interactions are likely an important source of waves at a range of scales not directly linked to source scales at lower altitudes. Because of their roles in spectral energy transfers and amplitude constraints, a more complete discussion of wave-wave interactions is deferred until section 6.1.

\subsection{Propagation Issues}

[54] Gravity wave propagation and inferences of gravity wave effects are complicated by a number of factors in the middle atmosphere. Spatially variable wind and stability profiles cause wave refraction, reflection, focusing, and ducting, while temporally variable winds alter wave phase speeds. Instability processes contribute to the generation of turbulence which may interact with or act as a source for additional wave activity. Gravity wave-vortical mode interactions may also exchange energy among these components of the motion spectrum. Our purpose in this section is to identify areas where these dynamics may confuse or obscure the inference of gravity waves and their effects in observational analyses.

\subsubsection{Upward and Downward Propagation}

[55] It is useful for many purposes to distinguish what fractions of the gravity wave spectrum are propagating upward and downward. Two approaches have usually been used to do this. One employs 2-D frequencyvertical wave number spectra or direct measurements of phase progression for individual waves to make the distinction, while a second employs a rotary spectral analysis of individual profiles. Each method is hampered by certain assumptions but yields insightful, if not precise, results.

[56] The 2-D spectral approach employed by Fukao et al. [1985], Fritts and Cho [1987], and Wilson et al. [1991a] assumes that observed and intrinsic frequencies are the same, thus confusing vertical directionality for motions having intrinsic phase speeds comparable to and opposed to mean winds and erring in the assignment of wave frequency. These are not generally serious errors when mean winds are not extreme because the total wave variance at small intrinsic phase speeds (hence small vertical scales and amplitudes) is not large. The 
forms of the observed frequency spectra of horizontal velocity and temperature (slopes typically near $-5 / 3$ ) do not change dramatically for mean winds smaller than the intrinsic phase speeds of the most energetic waves [Fritts and VanZandt, 1987], however, while there is relatively less variance for $m \sim N / \bar{u}_{h}$ for which Doppler-shifting effects are larger. These and other observations suggest that the fraction of upward propagating gravity wave energy is much greater than $1 / 2$ and that the upward propagating fraction is likely an underestimate because of uncertainties over wave frequencies [Vincent, 1984b; Fukao et al., 1985; Fritts and Chou, 1987; Wilson et al., 1991a; Lintelman and Gardner, 1994; Gavrilov et al., 1996; de la Torre et al., 1999].

[57] The second method, rotary spectral analysis, was first employed by Vincent [1984b] and is related to Stokes analysis used to assess the degree of polarization of the motion field [Vincent and Fritts, 1987; Eckermann and Vincent, 1989; Eckermann and Hocking, 1989; Barat and Cot, 1992; Nakamura et al., 1993a; de la Torre et al., 1999; Zink and Vincent, 2001a]. Rotary spectral analysis decomposes all oscillatory motions in the vertical into a superposition of upward and downward propagating motions assumed to be circularly polarized. This assumption causes a single upward propagating inertiagravity wave with amplitude $A$ and intrinsic frequency $\hat{\omega}>f$ (having elliptical polarization) to be misidentified as a sum of an upward propagating wave of amplitude $A_{u}=(1+f / \hat{\omega}) A / 2$ and a downward propagating wave of amplitude $A_{d}=(1-f / \hat{\omega}) A / 2$. As $\hat{\omega} \rightarrow f$, this yields $A_{u} \rightarrow 1$ and $A_{d} \rightarrow 0$ because the dynamics are then consistent with the assumptions. However, for $\hat{\omega}>f$ the inferred (and errant) downward propagating fraction of the total variance of $1 / 2-f \hat{\omega} /\left(\hat{\omega}^{2}+f^{2}\right)$ approaches $1 / 2$ as $f / \hat{\omega} \rightarrow 0$ (or as $\hat{\omega} \rightarrow N$ ) and is entirely errant [Eckermann and Vincent, 1989]. Thus the rotary spectral analysis likewise leads to a conservative estimate of the upward propagating fraction of gravity wave energy. Effects of wave superposition, while not always significant, may also influence the results of a Stokes analysis and contribute to a lack of precision in partitioning of wave energy between upward and downward propagating components [Eckermann and Hocking, 1989]. Studies that use the technique of ellipse fitting to hodographs [Tsuda et al., 1994a; Hamilton and Vincent, 1995; Shimizu and Tsuda, 1997] yield unambiguous estimates of the upward and downward propagating fractions, at least when a single dominant inertia-gravity wave is present in the profiles.

\subsubsection{Vertical Propagation Versus Ducting}

[58] Another issue that has arisen recently because of its implications both for estimates of gravity wave fluxes of heat and momentum and for the parameterization of gravity wave effects in large-scale models is the occurrence of gravity wave ducting. Waves that propagate vertically (with $\mathrm{m}^{2}>0$ ) but having reflection (or turning) levels above and below are trapped or ducted. From the dispersion relation, equation (24) or equation (33), we see that variations in the thermal structure, as measured by the buoyancy frequency $N$, influence waves having any direction of propagation in the same manner, whereas the wind profile affects waves having different propagation directions very differently. In general, wind maxima in a particular direction favor ducting at that level (and in that direction) because of the relative maxima they imply in $\mathrm{m}^{2}$, while maxima of $N^{2}$ favor ducting at this level for all propagation directions [Chimonas and Hines, 1986; Fritts and Yuan, 1989a]. Observational evidence of wind and thermal $\left(N^{2}\right)$ ducts was obtained by Isler et al. [1997] and Walterscheid et al. [1999], while Taylor et al. [1995] and Mitchell and Howells [1998] inferred ducting from limited vertical wave extent. In all these cases, gravity waves having horizontal wavelengths of $\sim 20 \mathrm{~km}$ or less were judged to have a high probability of being ducted by the wind and/or thermal structures of the atmosphere. The probability of ducting remains finite but decreases as horizontal wavelengths increase [Swenson et al., 2000].

[59] Gravity wave structure within a duct typically exhibits very little vertical phase variation across the duct and is evanescent on either side, though more complex wave structures are also possible. Thus typical motions are largely in phase and vertical, have intrinsic frequencies near $N$, may have only a gradual decay of wave amplitude away from the duct, and have little or no associated momentum flux. Yet because of their structure such motions may make large contributions to vertical velocity, temperature, and airglow intensity variances.

[60] Despite our appreciation of the implications of gravity wave ducting, there have been several efforts to associate airglow intensity and/or temperature variances with gravity wave momentum fluxes (to which ducted waves contribute nothing, according to linear theory). These efforts by Swenson et al. [1999] and Gardner et al. [1999a] have been critiqued by Fritts [2000] and are flawed because of their neglect of ducting. Swenson et al. [2000] likewise recognized the lack of sensitivity to ducting behavior in earlier formulations and discussed the implications of the more complete dispersion relation, reaching similar conclusions to those of Isler et al. [1997] and Walterscheid et al. [1999] regarding susceptible horizontal wavelengths. The implications of these studies are that turning levels and ducting are important processes that impact gravity wave structure and inferred effects where wind and thermal variations are significant, particularly for shorter horizontal wavelengths.

\subsubsection{Gravity Waves Versus Two-Dimensional Turbulence}

[61] While quasi-2-D turbulence (hereafter 2DT, also called geostrophic or stratified turbulence under related assumptions) clearly makes contributions to atmospheric dynamics and velocity variances at some altitudes, the role it plays in the middle atmosphere appears 
to be small, at least for scales well below synoptic and planetary scales $(\sim 500 \mathrm{~km})$ [Nastrom and Gage, 1985]. Gage [1979] and Lilly [1983] have advocated 2DT as an explanation for the $-5 / 3$ power law in lower stratospheric horizontal wave number spectra, and Lilly [1983] has suggested that deep convection may be a viable source for 2DT. However, subsequent assessments using radar and balloon data by Smith et al. [1985], Vincent and Eckermann [1990], and Eckermann and Vincent [1993] suggested that only a small fraction of horizontal velocity variance could not be attributed to gravity waves. Increasing energy and momentum fluxes (per unit mass) and energy dissipation rates with altitude appear, at first sight, to imply a reduced role at greater altitudes. Without a more complete understanding of middle-atmosphere turbulence dynamics and energetics, however, it would be a mistake to assume that no strong middleatmosphere sources of 2DT are present. This is particularly true given the potential role of gravity wavevortical mode interactions as wave amplitudes increase [Dong and Yeh, 1988; Yeh and Dong, 1989], the departures of ratios of kinetic to potential energy from those predicted by gravity wave theories [Tsuda et al., 1991; Nastrom et al., 1997; de la Torre et al., 1999], and the increasing scales and energies of turbulence with altitude (see section 4). We must be careful in attributing kinetic to potential energy ratios as evidence against a purely gravity wave interpretation, however, because such departures from theory can also arise from excess gravity wave energy near the inertial frequency [Thompson, 1978; Sato et al., 1999].

\section{SPECTRAL CHARACTER AND EVOLUTION}

[62] Models of the gravity wave spectrum have evolved with time as we have identified spectral characteristics and constraints more precisely. As described in section 6, various theories constrain the gravity wave spectrum to behave in a particular manner over some range of wave numbers and/or frequencies. These observational and theoretical constraints have led to a canonical gravity wave spectrum that offers insights into mean properties of the gravity wave field and its variations with altitude. We emphasize, however, that this canonical spectrum cannot capture the true complexity of the gravity wave field or its evolution in altitude. There are, in addition, many reasons to expect that the spectrum will also exhibit considerable variability spatially and temporally because of various sources, filtering environments, quasi-discrete waves, and gravity wave interactions with larger scales of motion. We first review in this section the form and implications of the canonical mean spectrum. We then discuss some of the causes of departures from the canonical spectral forms.

\subsection{Spectral Shape In $k, m$, and $\omega$}

[63] Frequency spectra of horizontal velocity and temperature typically vary as $E(\omega) \sim \omega^{-p}$ and exhibit slopes varying from $p \sim 1-2$ for frequencies between $f$ and $N$, with most slopes falling close to $p=5 / 3$ [see, e.g., VanZandt, 1982; Balsley and Carter, 1982; Nakamura et al., 1993b; Collins et al., 1994]. Because horizontal wind and temperature variances tend to be concentrated near inertial frequencies, Doppler effects are small, and the intrinsic and Doppler-shifted (i.e., ground based) frequency spectra are expected to have nearly the same forms [Scheffler and Liu, 1986; Fritts and VanZandt, 1987]. This expectation is supported by the limited Lagrangian measurements that are available [Quinn and Holzworth, 1987; Hertzog and Vial, 2001]. Ground-based frequency spectra of vertical velocity, on the other hand, exhibit considerable variability with altitude, primarily because these spectra are highly sensitive to Dopplershifting effects (see below).

[64] Vertical wave number spectra of velocities, temperatures, and various species concentrations have been measured with many instrument types. These spectra typically exhibit a distinct peak at the most energetic scales, with smaller power at lower and higher wave numbers. As noted above, a limited altitude range in observational data may result in a lack of sensitivity to motions with larger vertical scales. This causes those waves having larger vertical group velocities, thus potentially large energy and momentum fluxes, to be systematically underrepresented in such data sets. At the other extreme, resolution and measurement accuracy limits constrain the range of vertical scales that can be reliably measured. This suggests caution in assessments of characteristic scales, spectral slopes, and spectral amplitudes at large $m$. Nevertheless, multiple data sets suggest characteristic vertical wave numbers, $m_{*}$ (i.e., the vertical wave number of the most energetic gravity waves), corresponding to vertical wavelengths of $\sim 2-5 \mathrm{~km}$ in the lower stratosphere increasing to $\sim 10-30 \mathrm{~km}$ near the mesopause.

[65] Spectral slope estimates at high $m\left(m>m_{*}\right)$ also vary, but typical values range from -2.5 to -3 [Dewan et al., 1984; Tsuda et al., 1989, 1991]. Importantly, while there are departures from universal amplitudes and slopes (noted in section 4.2), particularly under strongly sheared conditions [Eckermann, 1995a], there is surprising conformity of spectral amplitudes and slopes at high $m$ with the expectations of the various saturation theories over a broad range of altitudes [Dewan and Good, 1986; Fritts and Chou, 1987; Smith et al., 1987; Fritts et al., 1988a; Sidi et al., 1988; Tsuda et al., 1989, 1990a; Wu and Widdel, 1989, 1990, 1991; Wilson et al., 1991a; Kuo et al., 1992; Collins et al., 1994; de la Torre et al., 1994; Allen and Vincent, 1995; Mitchell et al., 1996]. An example of the vertical wave number spectra of radial velocities exhibiting both the decrease of $m_{*}$ from the troposphere and stratosphere to the mesosphere and conformity to the spectral slopes expected for a satu- 


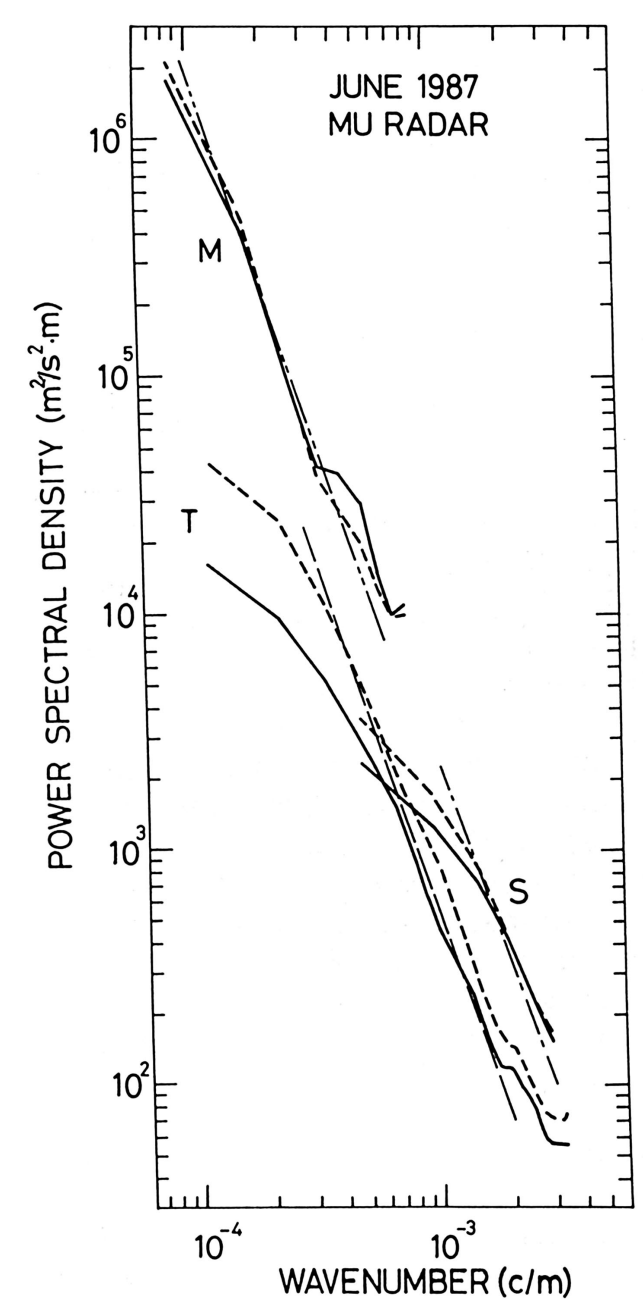

Figure 10. Radial velocity spectra assumed to represent largely horizontal velocities in the troposphere, stratosphere, and mesosphere (labeled T, S, and M) together with theoretical curves having amplitudes of $N^{2} / 6 \mathrm{~m}^{3}$. After Tsuda et al. [1989] (reprinted with permission of the American Meteorological Society).

rated gravity wave spectrum is shown in Figure 10 [Tsuda et al., 1989].

[66] Saturated or nonlinear gravity waves are not the only explanation offered for the observed spectral character, however; a recurring alternative is buoyancy-range turbulence [Weinstock, 1985; Sidi et al., 1988], which likely plays some role at the outer scales of turbulence accompanying initial instability processes. There is evidence, for example, that the ratio of kinetic to potential energy in the wave spectrum often exceeds that value predicted by the spectral model to be described later [Tsuda et al., 1991; Nastrom et al., 1997; de la Torre et al., 1999], perhaps suggesting other 2DT turbulence or quasi-inertial components of the motion field not included in the gravity wave spectral formulation. Spectral amplitudes also exhibit, on occasion, departures from canonical values which cannot be attributed to the measurement technique. These departures and some of the reasons for them are discussed at greater length in sections 4.2 and 4.3 .

[67] Horizontal wave number spectra are more challenging to measure unambiguously, as they mix spatial and temporal sampling. However, assuming that the speed of the measurement platform (typically, an aircraft) is large compared to wave horizontal phase speeds (this is not always true for the larger vertical scales), horizontal wave number spectra are obtained which are again in reasonable agreement with theory and other spectral measurements [VanZandt, 1982; Fritts et al., 1989, 1993a; Gardner et al., 1993; Bacmeister et al., 1996; Manson et al., 1998a]. Horizontal wavelengths range from $\sim 10 \mathrm{~km}$ to thousands of kilometers; typical slopes are $\sim-5 / 3$, and different dynamics appear to account for the spectral slope at larger horizontal scales [Nastrom and Gage, 1985; Nastrom et al., 1987; Jasperson et al., 1990].

[68] A spectral description that captures much of the observed character of the gravity wave field is assumed (largely for convenience) to be separable in intrinsic frequency and vertical wave number and is given by

$$
E(\mu, \omega, \phi)=E_{0} A(\mu) B(\omega) \Phi(\phi),
$$

where $E$ is the total gravity wave energy,

$$
\begin{gathered}
A(\mu)=A_{0} \frac{\mu^{s}}{1+\mu^{s+t},} \\
B(\omega)=B_{0} \omega^{-p},
\end{gathered}
$$

$\Phi(\phi)$ expresses the azimuthal distribution of wave propagation directions, $\mu=m / m_{*}$, and the coefficients are chosen such that $\int_{0}^{\infty} A(\mu) d \mu=1, \int_{f}^{N} B(\omega) d \omega=1$, and $\int_{0}^{2 \pi} \Phi(\phi) d \phi$ $=1$ [Fritts and VanZandt, 1993]. Typical values of the spectral slopes are $(s, t, p)=(1,3,5 / 3)$. The above form of the vertical wave number spectrum is more strongly peaked than was believed to be the case initially, while the requirement for a positive slope at small vertical wave numbers is imposed by the requirement for finite vertical energy fluxes (alternatively, one could impose a small- $m$ cutoff to achieve a finite energy flux). Of these parameters, the slope at small $m$ is perhaps the least well defined observationally or theoretically. The corresponding horizontal wave number spectrum can be derived from equations (45) and (46) and the gravity wave dispersion relation, equation (23); however, the overall spectrum in $(k, \omega)$ is then not separable. We also note that the spectrum given by equation (44) is specifically not separable in the presence of large-scale shears where vertical propagation implies differing evolutions for the $m$ spectrum at different azimuths [Fritts and $\mathrm{Lu}, 1993]$.

[69] The gravity wave spectrum is assumed to be constrained in amplitude at $m>m_{*}$ by saturation processes, with all of the various saturation theories (see section 6) yielding limits for kinetic and potential energy within a factor of 2 or so of 


$$
E_{k}\left(m \gg m_{*}\right) \simeq \frac{p N^{2}}{10 m^{3}}=\frac{N^{2}}{6 m^{3}}
$$

and

$$
E_{p}\left(m \gg m_{*}\right) \simeq \frac{N^{2}}{10 m^{3}} .
$$

Variations of total wave energy density, $E(m)$, with variations in altitude and $N$ are described approximately as [VanZandt and Fritts, 1989]

$$
\begin{aligned}
E(m) & =E(\mu) d \mu / d m \\
& =E_{0} A(\mu) / m_{*} \sim\left(N / N_{0}\right)^{1 / 2} e^{z / H_{E}} A(\mu) / m_{*},
\end{aligned}
$$

where

$$
m_{*} \sim\left(N / N_{0}\right)^{3 / 4} e^{-z / H_{*}}
$$

for $N$ increasing with height. Here $H_{E} \sim 2.3 H$ is a value consistent with observations and linear, fullspectral propagation simulations by Warner and McIntyre [1996]. Corresponding values of $H_{*}$ and $E_{0}$ are $H_{*} \simeq 2 H_{E}$ and $E_{0}=N^{2} / 10 m_{*}^{2}$. Equations (49) and (50) are also appropriate for $N$ decreasing with height and for variable $\bar{u}(z)$, provided that the amplitude decreases implied by these effects are compensated by amplitude increases resulting from density decreasing with altitude.

[70] The corresponding energy flux by the wave field, assuming that all motions propagate upward, is

$$
F_{E} \simeq \frac{N E_{0}}{18 m_{*}} .
$$

The factor $N / 18 m_{*} \simeq \overline{(1 / m)} \bar{\omega}$ is appropriate at midlatitudes (with $f / N \simeq 1 / 200$ ) and may be interpreted as an effective vertical group velocity $\bar{c}_{g z}$ [Fritts and VanZandt, 1993]. Then $m_{*} \sim 3 \mathrm{rad} \mathrm{km}^{-1}$ near the tropopause and $\sim 1 / 3 \mathrm{rad} \mathrm{km}^{-1}$ near the mesopause, with corresponding $\bar{c}_{g z} \sim 0.5$ and $5 \mathrm{~m} \mathrm{~s}^{-1}$, respectively, and model variances are consistent with numerous radar measurements [Vincent, 1984a; Fritts, 1984a, 1989; Balsley and Garello, 1985; Meek et al., 1985]. With $E_{0} \sim 10$ and $10^{3}$ $\mathrm{m}^{2} \mathrm{~s}^{-2}$, this implies $F_{E} \sim 5$ and $5 \times 10^{3} \mathrm{~m}^{3} \mathrm{~s}^{-3}$, respectively, near the tropopause and mesopause.

[71] With the above variations in $E(\mathrm{~m})$ and $m_{*}$ and assuming, for simplicity, a constant $N$ and that all energy flux divergence leads to turbulence, the corresponding turbulent energy dissipation rate may be written [VanZandt and Fritts, 1989] as

$$
\epsilon \simeq \frac{N E_{0}}{18 m_{*}}\left(\frac{1}{H}-\frac{3}{2 H_{E}}\right),
$$

with a corresponding dissipation timescale [Fritts and Werne, 2000] of

$$
T_{d} \sim E_{0} / \epsilon \sim \frac{18 m_{*}}{N}\left(\frac{1}{H}-\frac{3}{2 H_{E}}\right)^{-1} .
$$

There are, of course, uncertainties in $E_{0}, m_{*}$, and the fraction of wave energy that is directly dissipated as a result of wave interaction and instability processes. There are also variations that accompany changes of $N$, $H_{E}$, etc. with altitude. Nevertheless, these expressions provide useful guidance and/or limits for gravity influences throughout the middle atmosphere. The decreasing timescale for wave energy dissipation with increasing altitude, $T_{d} \sim E_{0}^{-1 / 2} \sim e^{-z / 2 H_{E}}$, is of relevance to the discussion of instability processes in section 6 . The decrease with altitude implies a transition from a tendency for local dynamical $(\mathrm{KH})$ instabilities in the lower stratosphere to a tendency for local convective instabilities in the mesosphere and lower thermosphere [Fritts and Werne, 2000] (also see section 6).

\subsection{Spectral Universality, Separability, and Departures}

[72] Despite the apparent role of various saturation processes in constraining wave amplitudes at large $m$, departures from the slopes and amplitudes predicted by the various saturation theories are common. In the troposphere, amplitudes are often larger by factors of $\sim 2$ to 3 [Smith et al., 1987; Tsuda et al., 1989, 1991; Allen and Vincent, 1995]. In the stratosphere, amplitudes vary from those expected from saturation theory due to wave filtering in mean shears [Eckermann, 1995a], enhanced saturation due to increasing $N$ with altitude [VanZandt and Fritts, 1989], or other effects not yet quantified [Cot and Barat, 1990; Senft and Gardner, 1991; Marsh et al., 1991; Tsuda et al., 1991]. Spectral amplitudes may also exceed saturation values at any altitude (but especially in the mesosphere and lower thermosphere) where large vertical group velocities impose wave amplitude growth that is more rapid than the finite time required for instability dynamics to impose wave amplitude constraints (see section 6).

[73] Though departures from canonical frequency spectra of horizontal velocities or temperature occur in many, if not most, observations, the degree of uniformity in frequency spectra of horizontal velocity and temperature is initially surprising. The relative lack of variability of these spectra is due in part to the occurrence of the majority of the variance at (intrinsic and observed) frequencies near $f$ and vertical wave numbers near or less than $m_{*}$. Large variances near $f$ imply vertical scales near $m_{*}$ (to enable large wave amplitudes) and corresponding large intrinsic phase speeds. This is especially the case in the mesosphere and lower thermosphere where $m_{*}$ is small, vertical scales are large, and intrinsic phase speeds are often comparable to or exceed mean winds. In this case, observed frequencies cannot be Doppler shifted more than a factor of 2 or so away from their intrinsic frequency, and intrinsic and observed spectra bear a close resemblance [Scheffler and Liu, 1986; Fritts and VanZandt, 1987]. Even in the stratosphere, where vertical scales and intrinsic phase speeds are smaller, the variance is still largely dominated by 
waves with large intrinsic phase speeds and low frequencies, and therefore Doppler shifting is not severe. In contrast, variances occurring at intrinsic frequencies near $N$ are much smaller and may be Doppler spread more widely without significantly changing the shape of observed frequency spectra.

[74] The situation is quite different for frequency spectra of vertical velocity. For a frequency spectrum of horizontal gravity wave motions as described in section 4.1 , we expect from equation (23) an intrinsic frequency spectrum of vertical velocities to have a slope of approximately $2-p$ and a sharp peak (in variance content, $\left.\hat{\omega} E_{w}(\hat{\omega})\right)$ at frequencies just below $N$. Here large variances at high frequencies are associated primarily with (nonhydrostatic) wave motions having large $\mathrm{k} / \mathrm{m}$ (see equation (30)) and vertical velocities comparable to or larger than horizontal velocities. Hence mean winds are often much larger than intrinsic phase speeds, Dopplershifting effects may be large, and frequency spectra of vertical velocity exhibit significant variability and dependence on mean winds [Fritts et al., 1990a; Fritts and Wang, 1991; VanZandt et al., 1991]. Indeed, several studies using vertical velocity frequency spectra have shown a tendency for waves to be propagating preferentially against the local mean flow [Fritts and Wang, 1991; VanZandt et al., 1991], in agreement with inferences from momentum flux measurements.

[75] Apart from theories which couple wave number and frequency spectra increasingly at higher altitudes [Dewan, 1994, 1997], there are no known reasons why frequency spectra of horizontal gravity wave motions should be universal. Indeed, specific sources impart to the wave field their own spectral attributes (see section 3 ). The tendency toward a canonical frequency spectrum as altitude increases more likely reflects a combination of (1) averaging over many wave sources and wave field realizations, (2) the variations of intrinsic frequency accompanying wave propagation in wind shear, and (3) systematic nonlinear interactions among spectral components which increase in importance with altitude.

[76] There is no reason to suppose that the gravity wave spectrum is separable among any two variables on theoretical grounds. Nor is separability fundamental to any saturation theory, despite claims to the contrary [Gardner et al., 1999b]. Separability simply provides a convenient means of describing the dependence of gravity wave spectra on observed frequency, vertical wave number, and/or azimuth of propagation having some experimental justification [Smith et al., 1987; Fritts and Chou, 1987; Fritts and VanZandt, 1987; VanZandt and Fritts, 1989; Sidi et al., 1988].

[77] Finally, we note that estimates of gravity wave spectral parameters depend to some extent on the technique employed, as each technique has its own measurement biases and limitations. Thus care must be taken in interpreting and, in particular, intercomparing these estimates. Limited altitude coverage, limited temporal extent, spatial and temporal averaging, and windowing and linear trend removal additionally cause underestimates of spectral variance at lower wave numbers and frequencies [Lefrere and Sidi, 1990]. Geophysical variations of the mean wind and stability profiles with altitude likewise introduce biases in spectral amplitudes and slopes [VanZandt and Fritts, 1989; Eckermann, 1990, 1995a].

\subsection{Broad Spectrum or Discrete Waves?}

[78] Spectral characterization of the gravity wave field almost always gives the impression that the wave field is composed of many components at various scales. This is true even for spectra formed from a single time series or vertical profile [Dewan, 1979; Reid et al., 1988; Fritts et al., 1988a; Wu and Widdel, 1989, 1991; Allen and Vincent, 1995; Sato and Yamada, 1994]. There is ample evidence, however, that spectra are often composed of only a few waves of significant amplitude, lending support to the arguments advanced by Hines [2002a] (see section 6.1). Most vertical profiles of velocity or temperature exhibit in any altitude range a single dominant wave structure; the corresponding vertical wave number spectrum typically has $\sim 70 \%$ of the variance within a factor of 2 of the characteristic vertical wave number, $m_{*}$ (see equation (49)).

[79] Additional evidence for a discrete or "narrow spectral," rather than a "broad spectral" description of the local wave field comes from many studies. Airglow observations more often than not exhibit a single or very few wave structures extending over a few to many wavelengths [Taylor et al., 1991, 1995, 1998; Hecht et al., 1997; Nakamura et al., 1998; Swenson et al., 1999; Yamada et al., 2001].

[80] Airglow and noctilucent cloud (NLC) observations have also yielded evidence of gravity wave instability processes accompanying single or a few large-amplitude wave motions at specific spatial scales [Fritts et al., 1993b; Swenson and Mende, 1994; Hecht et al., 1997; Yamada et al., 2001]. Balloon, lidar, and rocket data provide evidence of large-scale overturning of apparently individual waves, or superpositions of a few largeamplitude waves [Shutts et al., 1988; Hecht et al., 1997; Goldberg et al., 1997; Williams et al., 2002]. Radar and optical estimates of gravity wave momentum fluxes provide enticing evidence of discrete, large-amplitude events [Riggin et al., 1997; Fritts et al., 2002] and suggest that such events may comprise a significant part of the mean forcing. Finally, Sato and Yamada [1994] found that a single wave approaching a critical level can yield a vertical wave number spectrum having a -3 slope, while Eckermann [1999] showed that such a spectrum can arise from as few as four discrete sets of wave scales. Thus we must be cautious of claims that the spectral character of the gravity wave field implies a broad mix of waves occupying a common volume, as this often appears not to be the case. 


\section{CLIMATOLOGIES OF GRAVITY WAVE ACTIVITY}

[81] Because global model studies are increasingly recognizing the need to describe gravity wave effects via parameterization, efforts to observe and understand the climatology of gravity waves is an area of active research. Climatology in this context refers to the geographical and temporal variations in gravity wave activity (specifically momentum fluxes) and variations in gravity wave characteristics (wavelengths, phase speeds, etc.). If the mechanisms for gravity wave generation were completely understood and if their sources were fully characterized, we could in theory perfectly describe the temporal and geographical variations in gravity wave occurrence along with the specific wave characteristics for a given set of meteorological conditions. The largest obstacle to accurate parameterization of gravity wave effects in the middle atmosphere would then be overcome. Unfortunately, we instead have a disjointed set of observations that sometimes agree with one another and other times do not. We also have idealized model studies of gravity wave sources which are often poorly constrained by observations. Where such constraints do exist, models and observations do not always agree. Progress is being made, but the global characterization of the climatology of gravity wave occurrence remains a daunting observational challenge.

[82] In the last 15 years there have been a number of compilations of observations of small-scale temperature and horizontal wind structure in the middle atmosphere, with these structures interpreted as gravity wave perturbations. These compilations include long-term observations at single sites from which seasonal and interannual variations can be observed, global observations over some more limited period of time, and regional observations showing both temporal and geographical variations. The reasonably constant appearance of vertical wavelength and ground-based frequency spectra described in section 4 can be deceptive because of the logarithmic axes plotted. In fact, the gravity wave climatology information that has appeared in the literature shows substantial variations with time, with height, as well as with geography.

[83] Part of the difficulty in inferring a climatology of gravity waves from the existing set of observations is related to the fact that gravity waves are phenomena that occur with a broad and multidimensional spectrum of characteristics. Each observation technique tends to be sensitive only to some portion of that spectrum while being almost totally insensitive to other portions. These observational limitations can themselves be responsible for the climatological patterns observed in the data because the vertical wavelengths, intrinsic frequencies, and phase speeds of gravity waves can be dramatically altered by variations in the background atmosphere [ $\mathrm{Al}$ exander, 1998]. These variations in gravity waves due to background atmosphere effects must then be separated from variations associated with gravity wave sources before we can achieve accurate interpretations of gravity wave climatological observations.

[84] Our understanding of the nature of gravity wave sources was summarized in section 3.1. In this section we summarize global patterns and seasonal variations of gravity wave variances and fluxes that have been reported using different observational techniques. We then review the theoretical studies that have helped to explain some of these observed variations and separate background atmosphere effects from source variations. Inferences thus gained about global variations in gravity wave sources are then summarized.

\subsection{Observations}

\subsubsection{Remote Sensing Measurements From Space}

[85] Observations from satellite platforms hold great promise for providing the kind of global coverage at frequent time intervals that is needed to understand gravity wave variability. However, gravity waves can have quite small vertical and horizontal scales and short periods. They can therefore be challenging to observe remotely from space. New techniques have emerged, yet each has been capable of observing only some fraction of the gravity wave spectrum that may be present in the atmosphere, while missing other portions of the spectrum.

[86] Infrared limb-viewing profiles like the Limb Infrared Monitor of the Stratosphere (LIMS) instrument [Gille and Russell, 1984] retrieve temperature profiles from 15 to $60 \mathrm{~km}$ at high vertical resolution $(\sim 1.5 \mathrm{~km})$. However, line-of-sight (LOS) integration effects and the spacing between profiles limit these data to observation of only longer horizontal wavelengths. Fetzer and Gille [1994] retrieved global fields of gravity wave temperature perturbations by subtracting Kalman-filtered temperature fields from unfiltered temperature profiles. The Kalman-filtered data included planetary waves with zonal wave number $\leq 6$. The difference field, assumed to be gravity wave perturbations, included information on waves with vertical wavelengths of $\sim 6-50 \mathrm{~km}$ and horizontal wavelengths longer than $\sim 200 \mathrm{~km}$. (Note that aliasing from shorter unresolved horizontal wavelengths was evident, but these represented a small fraction of the total gravity wave variance observed [Fetzer and Gille, 1994].) Fetzer and Gille [1994] noted that the temperature variances derived from these data are likely dominated by low-frequency inertia-gravity waves. Maps of the LIMS gravity wave temperature variance (Figure 11a) as a function of latitude and height showed a peak near the equator in the lower stratosphere below $20 \mathrm{~km}$ and a peak in the winter stratospheric jet above $20 \mathrm{~km}$. Seasonal variation above $40 \mathrm{~km}$ at the equator related to the semiannual oscillation (SAO) was also evident in the gravity wave variance.

[87] Maps of gravity wave temperature variance have been similarly derived from Cryogenic Infrared Spectrometers and Telescopes for the Atmosphere 


\section{LIMS: November 1978}

a) Fetzer and Gille [1994]

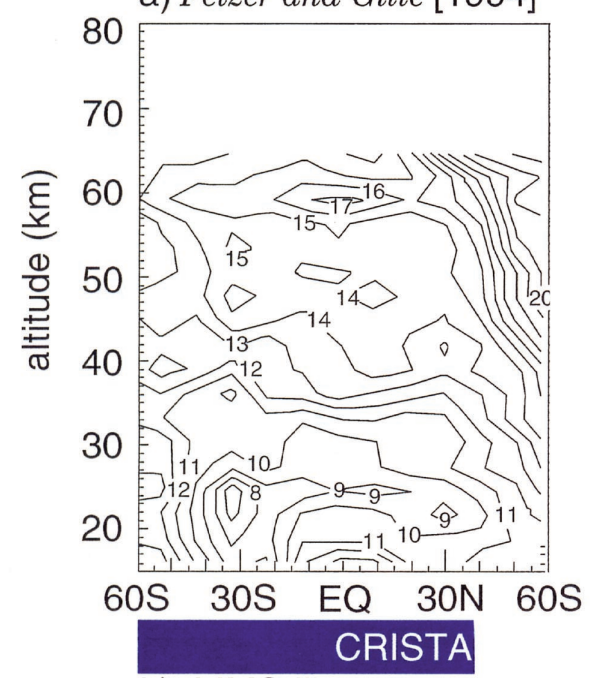

b) LIMS-like

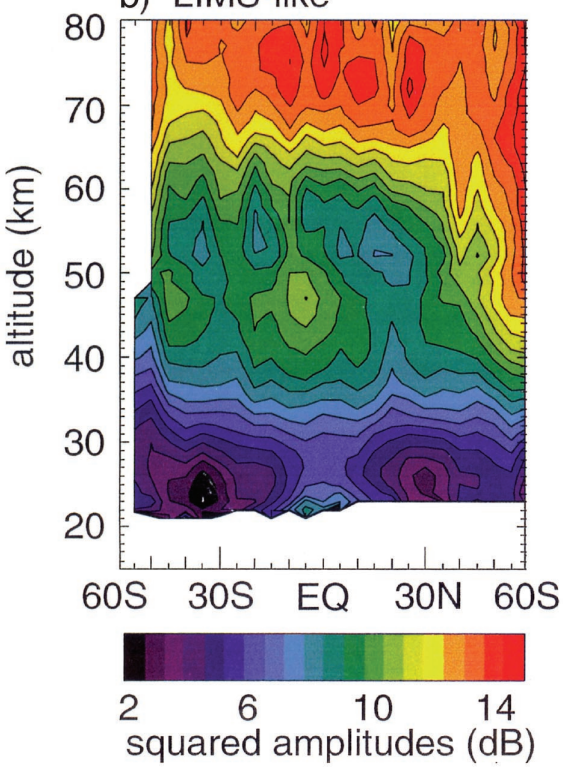

Figure 11. Comparison of zonal mean gravity wave temperature variance in November from (a) LIMS (after Fetzer and Gille [1994], reprinted with permission of the American Meteorological Society) and (b) CRISTA filtered to reflect the same vertical resolution as LIMS (after Preusse et al. [2000]).

(CRISTA) data [Preusse et al., 1999]. These data give temperature profiles at finer horizontal resolution and slightly better vertical resolution than LIMS. CRISTA's altitude range varies with observational mode but can extend from $\sim 20$ to $80 \mathrm{~km}$. The CRISTA data were used to infer that topography was the source for waves observed in the stratosphere from space [Eckermann and Preusse, 1999; Preusse et al., 2002]. Global maps of the gravity wave temperature variance show similar features as those from LIMS (Figure 11b).

[88] Tsuda et al. [2000] derived global maps of gravity wave potential energy derived from GPS occultation data temperature profiles [Rocken et al., 1997]. These observations include gravity waves with vertical wavelengths of $\sim 2-10 \mathrm{~km}$ in the 15 - to $40-\mathrm{km}$ altitude range. The LOS integration path length is also $\sim 200 \mathrm{~km}$, so the observed wave characteristics are similar to LIMS and CRISTA. The gravity wave energy shows a similar equatorial maximum below $\sim 25 \mathrm{~km}$ and midlatitude winter maxima at higher altitudes. At lower latitudes $\left(\leq 30^{\circ}\right)$, the gravity wave energy was particularly enhanced over regions of active convection. At midlatitudes, gravity wave energy was observed to be larger over continents than over oceans.

[89] Observations from the Microwave Limb Sounder (MLS) have been used to derive global maps of gravity wave temperature variance in the stratosphere and mesosphere [Wu and Waters, 1996a, 1996b]. These data include only gravity waves with long vertical wavelengths $\lambda_{z}>12 \mathrm{~km}$ because of the deeper weighting functions associated with microwave profilers compared to infrared profilers. One observation mode (the scanning mode) reportedly included gravity waves with horizontal wavelengths of $\sim 60-150 \mathrm{~km}$ [Wu and Waters, 1996a], while another (the tracking mode) included gravity waves with horizontal wavelengths up to $1000 \mathrm{~km}$. McLandress et al. [2000] noted that the integrated variance observed by the two modes is similar, suggesting that aliasing effects may be extending the range of wavelengths contributing to the variance in data from the scanning mode. Wu and Waters [1997] note that most of the observed waves propagate opposite to the direction of the wind. This, together with the long vertical wavelength restrictions, suggests these waves have much higher intrinsic frequencies than those observed via the other space-based observations described above. The global maps show peaks in variance at middle to high latitudes in winter, with a minor peak at subtropical latitudes in summer. Variances at the equator were indistinguishable from noise [McLandress et al., 2000]. Longitudinal variations suggest deep convection as a source of the waves observed in the summertime and topography as the source for waves at southern midlatitudes in winter [McLandress et al., 2000] (see also Figure 6).

\subsubsection{Radiosonde Profiles}

[90] Observations of temperature and horizontal wind profiles from radiosondes have provided information on short vertical wavelength gravity waves in the lower stratosphere below $\sim 25 \mathrm{~km}$. Several studies have inferred latitudinal, seasonal, and interannual variations in gravity wave activity in these data. The frequency of radiosonde launches rarely exceeds twice per day, so gravity wave perturbations are generally obtained by subtracting a low-order polynomial fit to the profiles and assuming the perturbations as a function of height are caused by gravity waves. In studies near the equator, however, where the inertial period is very long, radiosonde data have also been analyzed in the time domain 
for information on 1- to 3-day period oscillations as inertia-gravity waves [Sato et al., 1994; Sato and Dunkerton, 1997].

[91] Radiosonde profiles of horizontal wind plotted as hodographs generally show rotation indicative of upward propagating inertia-gravity waves [Ogino et al., 1995; Vincent and Alexander, 2000]. In fact, there is abundant evidence from analyses of radiosonde horizontal wind and temperature profiles that the perturbations in these vertical profiles are dominated by very low intrinsic frequency inertia-gravity waves, specifically those with frequencies between $\sim 1$ and $3 f$ [Vincent et al., 1997; Vincent and Alexander, 2000; Zink and Vincent, 2001a].

[92] Seasonal variations at northern midlatitudes and high latitudes show a maximum in gravity wave activity in winter and minimum in summer [Yoshiki and Sato, 2000; Kitamura and Hirota, 1989]. At very high northern latitudes, significant gravity wave variances were observed only in the absence of mountain wave critical levels [Bacmeister et al., 1990b; Worthington and Thomas, 1996; Whiteway and Duck, 1996; Whiteway et al., 1997]. At midlatitudes in the Southern Hemisphere, observations also show peak wave activity in winter [Allen and Vincent, 1995; Zink and Vincent, 2001a]. At Antarctic latitudes a maximum in spring has instead been observed [Allen and Vincent, 1995; Yoshiki and Sato, 2000].

[93] At the tropical latitudes of northern Australia, Indonesia, and the Indian Ocean a seasonal cycle in wave activity has been observed with a maximum in December to February [Allen and Vincent, 1995; Vincent and Alexander, 2000]. This is a period of intense deep convection in that part of the world. Waves observed in radiosonde data from that area also often tend to have long horizontal wavelengths, $1000 \mathrm{~km}$ [Tsuda et al., 1994a; Shimizu and Tsuda, 1997; Vincent and Alexander, 2000]. Propagation directions of gravity waves observed in radiosonde data at low latitudes are influenced by the winds of the quasibiennial oscillation (QBO). An excess of eastward propagating waves has been observed most prominently during periods with strong westward winds in the lower stratosphere and eastward shear aloft [Murayama et al., 1994; Tsuda et al., 1994a; Shimizu and Tsuda, 1997; Sato and Dunkerton, 1997; Vincent and Alexander, 2000]. Cadet and Teitelbaum [1979] observed the analogous situation in the opposite phase of the QBO: westward propagating gravity waves in westward QBO wind shear.

[94] Latitudinal variations are also apparent in gravity waves observed in radiosonde profiles. The data show a trend toward increasing wave energy equatorward of $\sim 30^{\circ}$ [Kitamura and Hirota, 1989; Allen and Vincent, 1995; Ogino et al., 1995]. Note that a similar equatorial maximum was observed in the GPS and IR limb sounding satellite observations in the lower stratosphere described above.

\subsubsection{Rocket Soundings}

[95] Rocket soundings of temperature and horizontal wind have been used to infer seasonal and latitudinal variations in gravity wave activity in the stratosphere below $\sim 60 \mathrm{~km}$ at $\sim 1-\mathrm{km}$ resolution. Rocket profiles from launches in the 1970s and 1980s at sites in the Northern Hemisphere and in the tropics have provided information on the seasonal and latitudinal variations in gravity wave activity in the stratosphere [Hirota, 1984; Hirota and Niki, 1985; Hamilton, 1991; Eckermann et al., 1995].

[96] The analysis method has varied between different studies, but the most common procedure is to subtract a background profile from the data and to assume that smaller-scale perturbations are gravity waves. In general, the rocket sounding analyses have shown a seasonal cycle in wave activity at high latitudes $\left(\sim 40^{\circ}-80^{\circ} \mathrm{N}\right)$ with a maximum in winter, similar to results of radiosonde analyses. Hodograph or rotary spectral analyses of rocket profiles have also shown a dominance of upward propagation, again similar to the radiosondes. At low latitudes, different analyses gave different seasonal cycles in wave activity, suggesting a sensitivity of this result to the vertical scales of waves considered. Mean kinetic and potential energies in the 20 - to $40-\mathrm{km}$ altitude range both showed increases at latitudes $<30^{\circ}$ [Eckermann et al., 1995]. A preference for eastward propagation at sites within $10^{\circ}$ of the equator has been noted as a possible indication that the wave activity at these sites is dominated by equatorial Kelvin waves [Hamilton, 1991; Eckermann, 1995b; Holton et al., 2001].

\subsubsection{Lidar}

[97] Lidar observations of middle atmosphere gravity waves have been restricted to a few specific sites. Among these, only a few have operated for long enough periods to provide information on seasonal variations in gravity wave activity [Wilson et al., 1991b; Marsh et al., 1991; Mitchell et al., 1991; Whiteway and Carswell, 1995]. These are all Rayleigh lidar studies of gravity wave temperature or density perturbations at midlatitude sites. The data include gravity waves with vertical wavelengths as short as $1 \mathrm{~km}$ at altitudes in the upper stratosphere and lower mesosphere. The data have been analyzed both for vertical variations as well as in the time domain, though these observations are limited to nighttime only. The dominant pattern that has appeared in monthly mean averages of these data is a seasonal cycle with maximum in winter and minimum in summer: the same annual cycle that is observed in radiosonde and rocket sounding data at midlatitudes.

\subsubsection{Radar}

[98] Radar observations of gravity wave wind perturbations can be made in the lower stratosphere and in the mesosphere and lower thermosphere. Many of these observations have been made at a limited number of sites and on a short-term campaign basis. We focus here 
on studies that have compiled observations over long enough time periods to study seasonal variations. These long-term studies have, to date, all been at midlatitude sites.

[99] Analyses of gravity wave observations in the mesosphere in the $\sim 65$ - to $90-\mathrm{km}$ region are generally reported for different ground-relative frequency bands for waves with periods ranging from 5 min to 24 hours. Gravity wave kinetic energy as well as variances $\left(\overline{u^{\prime 2}}, \overline{v^{\prime 2}}, \overline{w^{\prime 2}}\right)$ in each of the three component wind directions have shown a semiannual variation at these altitudes with maxima in summer and winter and minima in spring and fall [Meek et al., 1985; Vincent and Fritts, 1987; Tsuda et al., 1990a; Manson and Meek, 1993; Nakamura et al., 1996]. These analyses included observations at both Northern and Southern Hemisphere sites, and the results show similar gravity wave activity in the two hemispheres. A seasonal asymmetry in the occurrence of polar mesosphere summer echos (PMSE) and the ice particles that cause them has been observed. Specifically, there is an absence of PMSE in the Southern Hemisphere that may be a result of warmer temperatures in the south due to a weaker mean meridional circulation and corresponding weaker gravity wave forcing [Balsley et al., 1995; Woodman et al., 1999]. Some mesopause temperature measurements support this idea [Huaman and Balsley, 1999] while others suggest similar thermal structures at high latitudes in each hemisphere [Lübken et al., 1999]. A recent chemical-dynamical model study [Siskind et al., 2003] supports the idea of hemispheric asymmetries in gravity wave forcing and an asymmetric narrow warm layer at the mesopause that may be difficult to resolve with some measurement techniques.

[100] Observations of horizontal and vertical wind covariance $\overline{u^{\prime} w^{\prime}}$ (momentum flux per unit density) in the mesosphere have shown an annual cycle, positive (i.e., eastward) in summer and negative (i.e., westward) in winter, so the flux tends to have the opposite sign as the winds at these altitudes [Tsuda et al., 1990b; Manson and Meek, 1993; Nakamura et al., 1993c, 1996]. The anticorrelation between flux direction and the background wind direction is a condition observed even more generally in shorter-term data records [Vincent and Reid, 1983; Reid and Vincent, 1987; Fritts and Vincent, 1987; Reid et al., 1988; Fritts and Yuan, 1989b; Meyer et al., 1989; Wang and Fritts, 1990; Nakamura et al., 1993c]. The peak magnitudes of monthly mean fluxes (per unit mass) at mesospheric heights have been reported at $\sim 1-4 \mathrm{~m}^{2} \mathrm{~s}^{-2}$. Reports of meridional fluxes $\overline{v^{\prime} w^{\prime}}$ have been variable, so no repeatable seasonal cycle is known, but reported magnitudes are often comparable to or slightly larger than the zonal fluxes.

[101] Reports of seasonal changes in the anisotropy of wave propagation directions have varied among different studies [Manson and Meek, 1988; Nakamura et al., 1993d]. Maekawa et al. [1987] and Fan et al. [1991] inferred critical-level wind filtering as the mechanism responsible for anisotropies observed in the summer mesosphere wind field using the SOUSY and Arecibo VHF radars, respectively. Inferred propagation directions also depend greatly on the characteristics of gravity waves being observed. Vincent and Fritts [1987] and Nakamura et al. [1993b] observed a preference for meridional propagation of the dominant wave motions under winter conditions. Nakamura et al. [1993b] noted a tendency for propagation of the dominant motions toward either the NE or SW under summer conditions. Taylor et al. [1998] and Nakamura et al. [1998] observed propagation preferentially toward the NE for waves of shorter horizontal scales under summer conditions. Finally, Nakamura et al. [1993a] observed similar character in propagation directions at Adelaide and the MU radar at Kyoto in summer but with more isotropic propagation at Adelaide and more zonal propagation at Kyoto in winter.

[102] Observations in the stratosphere of the seasonal cycle of gravity wave activity measured by radar have been reported only for the MU radar near Kyoto in Japan for altitudes below $24 \mathrm{~km}$ [Tsuda et al., 1994b; Murayama et al., 1994; Sato, 1994]. These have shown an annual cycle in gravity wave variances $\left(\overline{u^{\prime 2}}, \overline{v^{\prime 2}}, \overline{w^{\prime 2}}\right)$ in all three wind components with minima in summer and maxima in winter to early spring. Sato [1994] and $\mathrm{Mu}$ rayama et al. [1994] also reported zonal and meridional momentum fluxes. The zonal component showed a clear annual cycle with large negative values $\sim-0.1 \mathrm{~m}^{2} \mathrm{~s}^{-2}$ in winter and near zero in summer. The meridional component also tended to be negative but with smaller magnitudes and no clear annual cycle. The observed wave characteristics at this site have been generally consistent with topographic gravity wave generation. Specific campaigns have, however, also observed waves associated with convection (see section 3.1).

\subsubsection{Aircraft}

[103] Measurements of wind and temperature fluctuations by aircraft flying in the stratosphere have yielded information on the horizontal wavelength spectrum of gravity waves [Nastrom et al., 1987; Bacmeister et al., 1996] (see section 4.1). Some aircraft studies have related gravity wave characteristics in the stratosphere to the wave sources below, including mountains [Gary, 1989; Bacmeister et al., 1990a, 1990b; Nastrom and Fritts, 1992; Leutbecher and Volkert, 2000] and convection and frontal systems [Fritts and Nastrom, 1992; Pfister et al., 1993a, 1993b; Alexander and Pfister, 1995; Alexander et al., 2000]. Several of these studies have provided in situ determination of gravity wave momentum fluxes. However, aircraft observations have been too limited in their duration to provide much information about the gravity wave climatology directly. Instead, they have contributed to our understanding of gravity wave variability by providing important quantitative constraints for theoretical and model studies of gravity wave sources (see section 3.1 and Figure 9). 

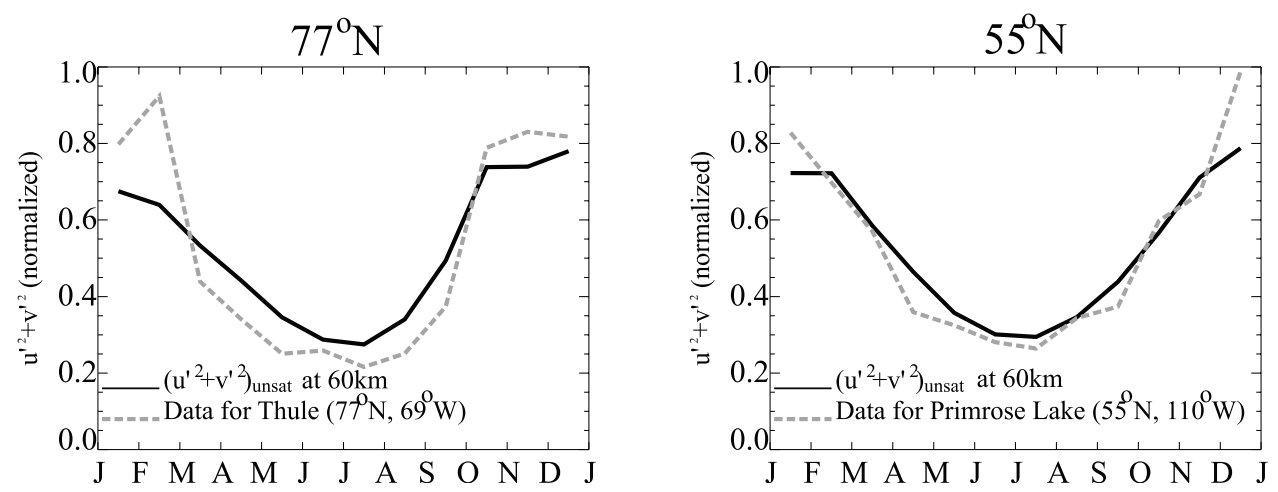

Figure 12. Seasonal variations observed in gravity wave horizontal wind variance in rocket soundings (dashed lines) and theoretical variations given by (54). The dotted line represents the seasonal variation that would be predicted if the wave amplitudes were saturated. After Eckermann [1995b] (reprinted with permission of Elsevier Science).

\subsection{Theoretical Studies of the Global Patterns Observed in Gravity Waves}

[104] At middle to high latitudes throughout the stratosphere the long-term measurements of gravity wave variances cited have shown an annual cycle with maximum in winter and minimum in summer. Eckermann [1995b] described a simple linear theoretical model that explained the annual cycle in both lidar and rocket sounding observations without any seasonal variation in gravity wave sources. This linear theory describes the change in amplitude of a gravity wave propagating vertically without dissipation from a source level $z_{0}$ where amplitudes are presumed constant to the observation level $z$. The theory predicts a simple proportionality for horizontal wind variance,

$$
\overline{u^{\prime 2}} \propto\left(N / N_{0}\right)\left(\rho_{0} / \rho\right),
$$

and for temperature variance,

$$
\overline{\left(T^{\prime} / \bar{T}\right)^{2}} \propto\left(N^{3} / N_{0}\right)\left(\rho_{0} / \rho\right),
$$

that fit the observed annual cycles in variance quite well (Figure 12) despite the fact that shear effects are neglected. The theory yields larger variances in winter because the colder winter stratosphere (with correspondingly smaller-scale height and faster decrease in density) leads to faster amplitude growth with height. Note that the factor depending on stability $N$ is more important for explaining temperature variance $\left(\propto N^{3}\right)$ than wind variance $(\propto N)$. Equations (54) and (55) may also be important for explaining the seasonal variations seen in radar and GPS observations. Note that in equations (54) and (55), $z$ is the geometric altitude. For observations reported on pressure altitude levels $z_{p}$ $=H \ln \left(p_{0} / p\right)$, such as radiosonde data, the density factor will be unimportant, and the effect greatly reduced.

[105] Seasonal and latitudinal variations observed in MLS gravity wave temperature variance were largely explained without variations in gravity wave sources by Alexander [1998]. Background wind effects were shown to have a controlling influence on the patterns in MLS gravity wave activity. Alexander [1998] showed how the MLS observation method essentially applies a low-pass filter to the vertical wave number spectrum of waves in the atmosphere that tends to allow the MLS to observe waves only at those places in the atmosphere where the background winds are very strong. In the stratosphere, these places are in the winter polar jet and in the summer subtropical jet. McLandress et al. [2000] further found that the waves seen by MLS in the winter hemisphere jet have, on average, smaller intrinsic phase speeds $\left(\sim 20 \mathrm{~m} \mathrm{~s}^{-1}\right.$ or less), while those in the summer hemisphere subtropics tend to have larger intrinsic phase speeds $\left(\sim 50 \mathrm{~m} \mathrm{~s}^{-1}\right.$ or less). This result is consistent with topographic sources being more important at high latitudes and deep convective sources being more important at low latitudes. Alexander [1998] also showed that seasonal changes in background winds can give rise to the late summer peak in variance that was observed in rocket sounding observations at low latitudes [Eckermann et al., 1995] without any seasonal changes in sources.

[106] The December to February peak in wave activity observed in low-latitude radiosonde observations [Vincent and Alexander, 2000] was explained without any pronounced seasonal variation in wave sources in a companion model study [Alexander and Vincent, 2000]. However, the spectrum of waves input into their model required certain characteristics. The waves input needed to have slow phase speeds, $<5 \mathrm{~m} \mathrm{~s}^{-1}$ relative to the ground, and the source level had to be in the upper troposphere or at the tropopause. This is consistent with the spectrum of waves that would be generated if the source were slow-moving deep convective complexes that generated waves via the obstacle effect near the tropopause analogous to topographic wave forcing [Clark et al., 1986; Pfister et al., 1993b]. There is also a weak annual cycle in cloud top height near Cocos Island [Vincent and Alexander, 2000] that may imply some contribution to the gravity wave annual cycle due to source variations. 
[107] GPS, CRISTA, radiosonde, and rocket sounding data have all shown a latitudinal variation in gravity wave energy in the lower stratosphere with increasing variance equatorward of $\sim 30^{\circ}$. Alexander et al. [2002] proposed this peak is associated with the latitudinal variation in the Coriolis parameter $f$ which defines the lowest gravity wave intrinsic frequency as a function of latitude. Each of these four observational techniques is sensitive to these low-frequency waves. The linear theory they applied to explain the peak accounts for the dependence of gravity wave vertical group velocity on intrinsic frequency (see equation (39)). If there is intermittency in the forcing of waves in the equatorial region (such as would be expected of waves generated by convection), then the slow vertical group velocities of the low-frequency waves make them the most probable waves to be observed. The theory then accounts for the nearly ubiquitous presence of low intrinsic frequency waves at low latitudes and also accounts for the low-latitude peak seen in long-term averages of gravity wave energy cited above. The results of Alexander et al. [2002] further imply that although higher-frequency waves appear relatively unimportant in the monthly mean zonally averaged gravity wave energy, they may dominate the gravity wave momentum flux spectrum.

[108] Seasonal variations in zonal momentum flux $\overline{u^{\prime} w^{\prime}}$ observed by radar in the midlatitude mesosphere, showing the mean flux opposes the background wind (positive in summer and negative in winter), are qualitatively consistent with our understanding of how the background winds would filter the gravity wave spectrum [e.g., Maekawa et al., 1987; Fan et al., 1991]: Summer westward winds would prevent most of the westward propagating waves from reaching the mesosphere, leaving primarily eastward propagating waves with positive momentum flux, and the reverse would be true in winter. Fluxes were observed to be small at equinoxes, but this is probably not due to averaging of large negative and positive fluxes together. Rather, the small equinoctal fluxes and kinetic energies, taken together, suggest that wave amplitudes are simply smaller at equinoxes. The equinoctal minima may be directly associated with the weak mesospheric winds at these times rather than any seasonal variation in sources. In weak wind conditions, waves are not Doppler shifted to high intrinsic phase speeds, and this results in smaller-amplitude limits inferred from linear instability theory (see section 6).

\subsection{Summary: Global Characteristics of Gravity Waves and Their Sources and Variability}

\subsubsection{Sources}

[109] There is convincing evidence for mountain waves in the stratosphere with fair quantitative agreement between high-resolution model studies and observations. Parameterized mountain waves, however, have a large effect in global models, and improved constraints on their properties and intermittency in occurrence are still needed. There is convincing evidence for convectively generated gravity waves in the stratosphere with qualitatively good agreement between observations and models. Observations that include both detailed information about the storm characteristics and about the waves that are generated are hard to find but are needed to make our understanding of convectively generated gravity waves more quantitative. There is evidence for low-frequency waves emanating from frontal zones in the jet stream and high-resolution model studies describing inertia-gravity wave generation under these conditions. Observational evidence detailed enough for a quantitative understanding of this wave source is lacking. A detailed understanding of the generation mechanisms and the resulting wave characteristics is needed for accurate description of gravity wave effects in global models.

\subsubsection{Variability}

[110] Observations of short vertical wavelength gravity waves in the lower stratosphere show increases in variance at latitudes $<30^{\circ}$. This has been interpreted as evidence for very low intrinsic frequency waves at low latitudes that are excluded from higher latitudes by the variation in $f$ and that have intermittent sources in time, such as would be expected from convection. Very close to the equator, at latitudes $<10^{\circ}$, these measurements could also include contributions from short vertical wavelength Kelvin waves. Also in the tropics, there is evidence for geographical variations in long vertical wavelength gravity waves with high intrinsic phase speeds that are closely tied to deep convection. These observations support model studies that show how deep heat sources generate long vertical wavelength waves.

[111] The evidence for global variations in gravity wave sources from climatologies of gravity wave activity is currently weak because many of the most prominent variations observed can be explained by background atmosphere effects without any seasonal or geographical variations in sources. Yet we have clear evidence for the importance of topography, convection, and frontal generation of gravity waves, and these sources must clearly vary seasonally and geographically. The existing gravity wave climatologies that describe monthly mean gravity wave kinetic and potential energy have largely served their purpose. They have allowed us to test the linear theory of gravity waves and their interaction with the background atmosphere.

[112] Observational gravity wave studies in the future must begin to define the deviations in gravity wave activity from these climatological patterns. Observations and modeling tools must be used together to separate these variations due to the background atmosphere and observational biases from variations due to sources. Quantitative measures of the intermittency in wave activity are needed along with any monthly mean quantity since it is important to define whether a monthly mean 
value represents intermittent large bursts of wave activity or more constant, smaller-amplitude wave activity.

[113] Satellite observations hold the most promise for providing the kind of geographical and temporal coverage needed to understand gravity wave variability. However, inherent observational limitations mean that continued in situ measurements from aircraft and highresolution ground-based profiling instruments will still be needed to understand the full spectrum of gravity waves present in the atmosphere.

\section{INTERACTIONS, INSTABILITY, AND SATURATION}

[114] Nonlinearity is a ubiquitous aspect of gravity wave dynamics. At smaller wave amplitudes, nonlinearity leads to slow energy transfers via three-wave interactions satisfying resonance conditions for wave frequencies and wave number vectors. At larger amplitudes, multiple-wave, nonresonant, and wave-vortical mode interactions become possible, culminating in the local instability of the wave via generalized wave breaking or shear instability processes as amplitudes increase further. Wave-mean flow interactions may also play important roles at larger wave amplitudes, altering wave propagation, inducing instability, and/or leading to excitation of additional waves. Thus nonlinearity has pervasive influences and is always poised to intercede more vigorously when linear dynamics imply excessive wave amplitudes. A number of reviews of the earlier developments in these areas are available; several relevant to gravity wave instability processes include Fritts and Rastogi [1985], Müller et al. [1986], Thorpe [1987], and Dunkerton [1989].

[115] More recent theoretical, numerical, and laboratory studies have expanded our understanding of gravity wave instability processes considerably. Several studies have begun to clarify the diversity of instability character with wave amplitude and propagation angle, including identifying links between the resonant interactions arising at small wave amplitudes and the local instabilities accompanying wave breaking and turbulence generation at large amplitudes [Klostermeyer, 1991; Lombard and Riley, 1996; Sonmor and Klaassen, 1997; Dunkerton, 1997a]. Other efforts have demonstrated the role of wave-wave interactions at small and large wave amplitudes [Dunkerton, 1987; Klostermeyer, 1991; Thorpe, 1994; Vanneste, 1995] and defined the dynamics of the transition to turbulence for gravity waves that are locally convectively or dynamically unstable [Winters and D'Asaro, 1994; Andreassen et al., 1994, 1998; Fritts et al., 1994, 1996a, 1998; Fritts and Werne, 2000]. Further studies have addressed the processes accounting for more general spectral energy transfers, effects of finite amplitude and wave-mean flow interactions on wave propagation, dispersion, and instability, and the competition among and finite-amplitude responses to initial instabil- ities of varying amplitudes. Our purpose in this section is to review the more recent work in these areas.

\subsection{Wave-Wave Interactions}

[116] Wave-wave interactions as a source of middle atmosphere gravity waves were discussed briefly in section 3. The purpose here is to review their expected influences on the shape and evolution of the gravity wave spectrum more broadly. A number of authors have addressed wave-wave interactions and spectral evolution from a statistical perspective; others have approached the problem deterministically. There remain, however, substantial uncertainties and disagreements over the roles these interactions play in spectral evolution and wave amplitude constraints.

[117] Early studies of wave-wave interactions in the atmosphere identified the dominant interactions previously recognized in oceanic applications [McComas and Bretherton, 1977; Yeh and Liu, 1981; Müller et al., 1986]. Three-wave (second order) resonant interactions require a match of wave numbers and frequencies among the participating waves satisfying

$$
\mathbf{k}=\mathbf{k}_{\mathbf{1}}+\mathbf{k}_{\mathbf{2}}
$$

and

$$
\hat{\omega}=\hat{\omega}_{1}+\hat{\omega}_{2},
$$

where subscripts denote the secondary wave quantities, and include (1) elastic scattering, (2) induced diffusion, and (3) parametric subharmonic instability (PSI). Elastic scattering refers to the backscattering of an upward propagating wave into a downward propagating wave (or vice versa) of comparable vertical wave number by a low-frequency motion (or mean flow) having twice the vertical wave number (Bragg scattering). Induced diffusion refers to the transfer of energy from one wave to another having a nearly identical wave number through interaction with a low-frequency (or mean) structure at a much smaller vertical wave number. Alternatively, induced diffusion may be regarded as the evolution of a wave packet in a large-scale shear flow. The third interaction, and arguably the most important in terms of spectral broadening or wave amplitude constraints, is PSI. In its most studied form, PSI represents an exchange of energy from a dominant motion of intermediate frequency to two motions having approximately half the parent frequency and large and nearly opposite wave number vectors, though other wave number combinations exhibit preferred growth in certain circumstances, such as the presence of a mean shear.

[118] More recent efforts have identified other possibilities for energy exchange and further modes of interaction likely to occur among atmospheric waves and other motions. Broutman and Young [1986] and Broutman et al. [1997], for example, noted that an energetic resonant-triad member can result in an irreversible transfer of energy to small wave numbers not anticipated 
by McComas and Bretherton [1977]. Dong and Yeh [1988] relaxed the assumptions of Yeh and Liu [1981] and considered wave-wave interactions not confined to a vertical plane or a resonance surface (nonresonant interactions were also discussed by Müller et al. [1986]). Further efforts revealed a potential for nonresonant gravity wave-vortical mode interactions for which the threshold amplitude is reduced or removed in the presence of rotation [Yeh and Dong, 1989]. As wave amplitudes increase, additional interactions become energetically viable. Dunkerton [1987] performed numerical studies to assess the impact of wave-wave interactions on wave momentum transport, with and without mean shear. He found a rapid energy transfer on the timescale of the forced waves following attainment of large amplitudes, followed by a nonlinear cascade toward increasing complexity as additional interactions became possible. When critical levels were present for the forced waves, Dunkerton [1987] found weaker resonant wave-wave interactions, the occurrence of additional nonresonant interactions, and the excitation of a wave having higher intrinsic frequency than the forced waves corresponding to a different branch of the PSI.

[119] Klostermeyer [1991] likewise found that larger primary wave amplitudes enable a cascade of interactions that quickly populate the spectrum, while waves that approach or exceed a convectively unstable amplitude support various modes of instability [Hines, 1971, 1988b; Lombard and Riley, 1996; Sonmor and Klaassen, 1997; Dunkerton, 1997a]. Vanneste [1995] performed both analytic and numerical studies of wave-wave interactions localized in space and noted good agreement between the two methods. In particular, Vanneste [1995] found that spatially localized interactions transferred significant energy on a timescale of $\sim 10$ primary wave periods for a primary wave amplitude of $a$ $=u^{\prime} / c \sim 0.25$. Vanneste [1995] also noted that mean shear suppresses the dominant unsheared wave-wave interaction but enables a "difference instability" which occurs near the primary wave critical level and has a secondary wave frequency larger than that of the primary wave (like Dunkerton [1987]), downward secondary wave propagation, and a growth rate that increases with shear strength. Such interactions are necessarily only resonant at specific locations but, nevertheless, enable rapid energy transfers under certain conditions [Grimshaw, 1988].

[120] An example of resonant excitation via PSI in the laboratory study by Thorpe [1994] is shown in Figure 13. Here a primary wave with phase aligned along the mean tilt of the tank transfers energy to a lower-frequency wave that becomes the predominant wave at later times. The wave-wave interaction proceeds quickly because of the large forcing wave amplitude, and the secondary wave achieves local convective instability. A numerical wave-wave interaction study performed by Vanneste [1995] is displayed in Figure 14 and exhibits the decay of a primary wave at higher frequency (Figure 14b) into two motions having lower frequencies (Figures $14 \mathrm{c}$ and $14 \mathrm{~d})$. The mean flow also experiences significant modifications in the presence of strong wave-wave interactions and can result in critical-level interactions and convective instability of the wave field [Sutherland, 2001]. Hence wave-wave and wave-mean flow interactions should be expected to play increasing roles in spectral evolution and energy and momentum fluxes as wave amplitudes increase with altitude.

[121] Theories of middle atmosphere spectral evolution employing statistical wave-wave interactions have also borrowed from the oceanic literature. The Lagrangian arguments by Allen and Joseph [1989] were employed in the Doppler spread theory by Hines [1991, 1993] to account for the form of the saturated "tail" spectrum. Hines [1996] extended these arguments and used the apparent success of this model to argue against the relevance of other existing saturation theories, most specifically "linear" saturation theory [Dewan and Good, 1986; Smith et al., 1987] and the "nonlinear diffusion" theory by Weinstock [1976, 1982, 1984, 1990]. The Hines theory attributes the large majority of spectral energy transfers to Doppler spreading by a "broad" spectrum of waves, with wave amplitudes and interactions increasing with altitude. The theory presumes that dissipation generally does not occur except at small scales having vertical wave numbers $m>m_{M}$, where $m_{M}$ is the maximum vertical wave number for which spectral character is determined largely by Doppler spreading, and infers a spectral shape approaching the $m^{-3}$ value most often cited by other saturation theories and various observations cited above. Advantages of the Hines theory include a recognition of the increasing statistical importance of nonlinear wave-wave interactions with increasing wave amplitudes and a natural transition from "linear" source spectra at lower altitudes to a nonlinear "saturated" spectrum at higher altitudes.

[122] There has been considerable confusion over the Allen and Joseph [1989] results and their implications for Doppler-spread theory over time. Hines [1999] noted an error in the Allen and Joseph analysis overlooked in his earlier papers and suggested a corrected high- $m$ spectral form of $\mathrm{m}^{-1}$ rather than $\mathrm{m}^{-3}$. More recently, Hines [2001] and Chunchuzov [2002] argued for the removal of the $m^{-1}$ term arising from the error correction to again infer a high- $m$ spectrum of the form $m^{-3}$ but with an amplitude $\sim 2-3$ times smaller than typically observed.

[123] The Hines Doppler spread theory has its detractors, however. Several authors have performed numerical studies that appear to seriously undermine the assumptions of Doppler spread theory [Zhong et al., 1995; Bruhwiler and Kaper, 1995; Broutman et al., 1997; Eckermann, 1997; Buckley et al., 1999; Walterscheid, 2000]. Essentially, these studies show that when time dependence and vertical motions of the underlying wave field are accounted for, the tendency for transfer of spectral energy to ever smaller vertical scales is substantially reduced (or reversed). Zhong et al. [1995] employed 


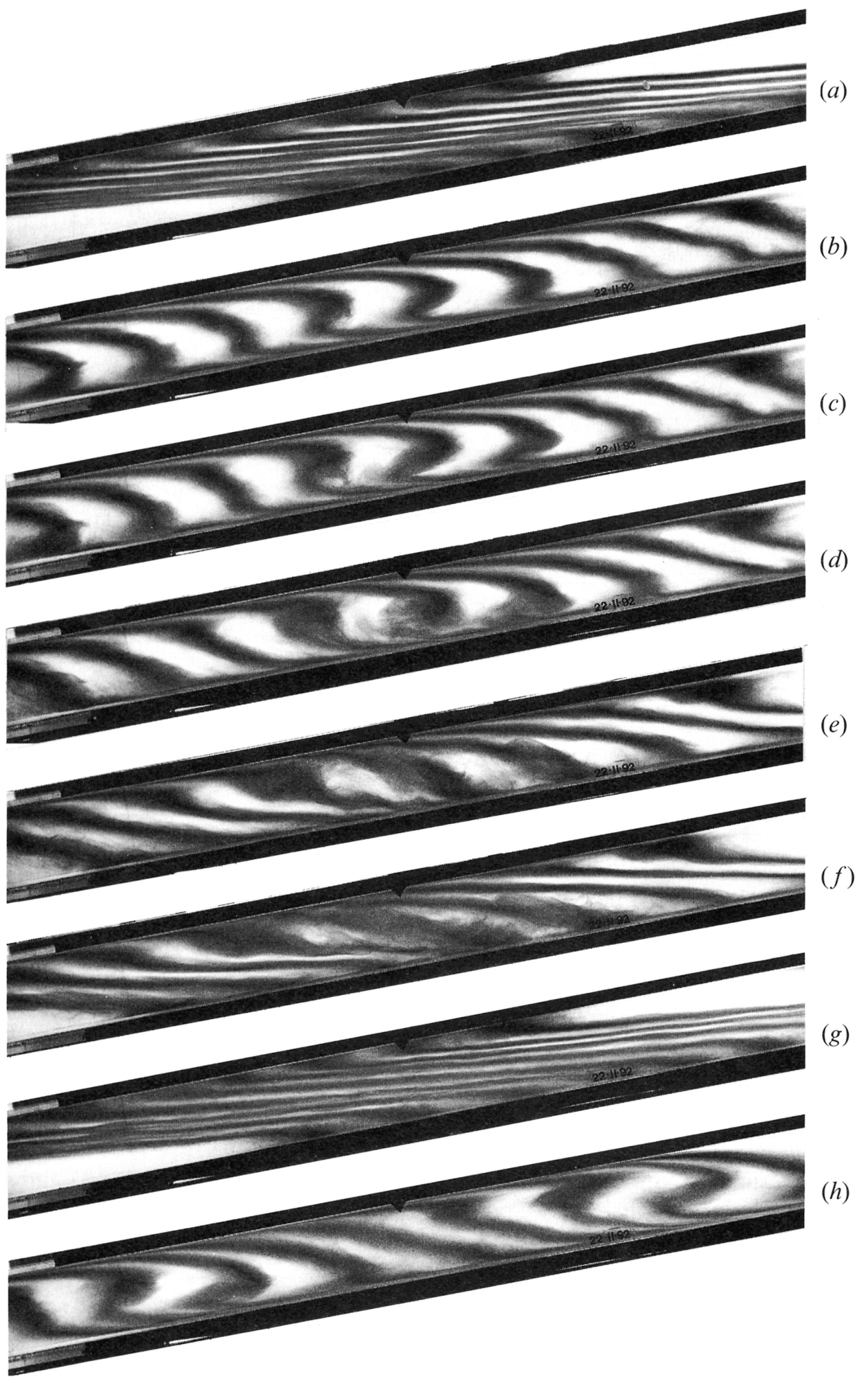

Figure 13. Dyed isopycnal surfaces in a rocked inclined tube showing the development and instability of a wave excited by the PSI. Note that the primary wave (with uniform structure along the tube) contributes largely at early times, whereas the subharmonic wave dominates the instability at later stages. After Thorpe [1994] (reprinted with permission of Cambridge University Press).

ray-tracing techniques to examine tidal modulation of wave propagation and noted that time dependence caused critical levels to become transient. Bruhwiler and Kaper [1995] formulated the problem from a Hamilto- nian perspective and showed that the theory was in good agreement with ray-tracing results for a spectrum of waves with various background wave amplitudes. They found, in particular, a tendency for low- $m$ waves to 


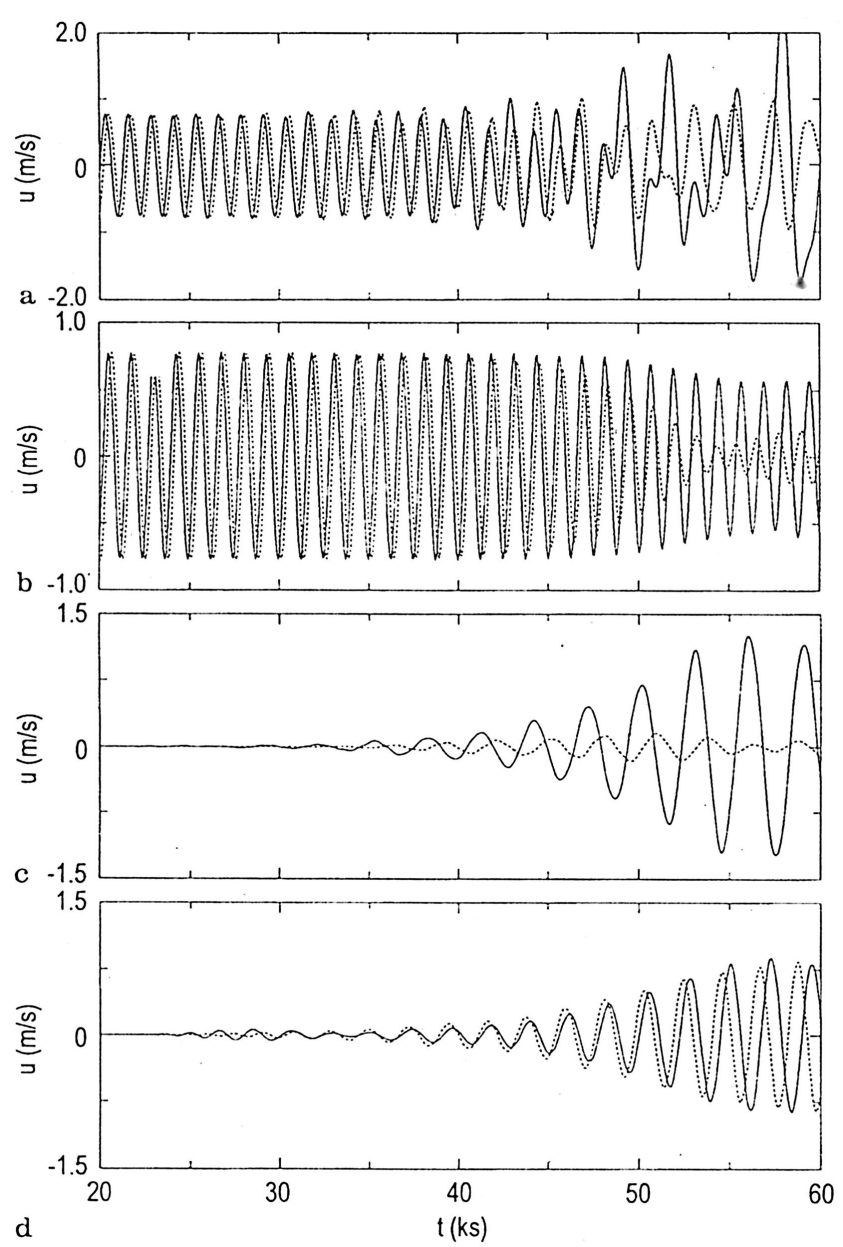

Figure 14. Numerical simulation of PSI in an unsheared atmosphere with a scale height of $7 \mathrm{~km}$. Shown are the (first panel) total, (second panel) primary wave, and (third and fourth panels) two secondary wave vertical velocities at altitudes of 10.7 (solid lines) and $17.75 \mathrm{~km}$ (dashed lines). Note that both secondary waves grow to amplitudes exceeding that of the primary wave as the interaction proceeds. After Vanneste [1995] (reprinted with permission of Springer-Verlag).

remain at low $m$ and for high- $m$ waves to move preferentially to lower $m$, representing a sharp departure from expectations based on Doppler spread arguments.

[124] Broutman et al. [1997] performed both ray-tracing and full numerical simulations, demonstrating the equivalence of the two approaches and the various influences of spatial and temporal variability on shortwave propagation. These results are illustrated in Figure 15. In particular, they exhibit a scattering of short-wave (large $m$ ) energy to both smaller and larger wave numbers, consistent with previous assessments based only on ray tracing [Broutman, 1986; Broutman and Young, 1986; Bruhwiler and Kaper, 1995], and a spectral form close to $\mathrm{m}^{-3}$ that is consistent with the results of Eckermann [1999]. Eckermann [1997] and Walterscheid [2000] also considered single waves in backgrounds having various character. Eckermann [1997] addressed specifically the various assumptions underlying the Doppler spread the- ory by Hines [1991, 1993, 1996], concluding that wave field transience significantly reduces the tendency to transfer wave energy to smaller vertical scales and eliminates a cutoff in unspread vertical wave numbers near $m_{c}$, itself a key element of Doppler spread theory. Walterscheid [2000] obtained similar results, emphasizing both the mitigating effects of time dependence on Doppler spreading and the role of vertical velocities in allowing wave packets to penetrate anticipated critical levels. Hines [1999] raised strong objections to the analysis by Eckermann [1997], arguing that the multiple-wave background offset the results of a single-wave background to a significant degree.

[125] Buckley et al. [1999] and Sonmor and Klaassen [2000] extended the analyses by Eckermann, Broutman et al., and Walterscheid by considering the effects of a spectrum of background waves, isolated wave packets, and a mean shear. Both studies suggest a weaker tendency for refraction toward large $m$ than with the steady assumptions underlying Doppler spread theory. Sonmor and Klaassen [2000] also found that multiple waves induce caustics under more general conditions than supported for single-wave environments, while both studies found that mean shears have significant cumulative refraction effects over timescales of a few inertial periods.

[126] Doppler spread theory, as it is presently developed, precludes competitive instability processes (i.e., linear saturation theory) from contributing to the shape of the vertical wave number spectrum except, to quote from Hines [1996], at wave numbers "a little less than $m_{M}$; but, arising as they almost certainly do in small-scale 'white-cap' regions, their effect is unlikely to extend very far". However, an increasing number of numerical and observational studies are providing evidence that local wave instability, via convective or shear instability, at large vertical scales is more the rule than the exception (see section 6.2.1), despite the clear potential for wavewave interactions to contribute importantly to energy transfers as wave amplitudes increase. Additional observations suggesting that the atmospheric wave spectrum is often not broad but is composed of a single or a few dominant motions further undermine the ability of Doppler spreading among multiple waves to provide large enough spectral transfers and to reproduce the observed amplitude limits.

[127] Clearly, the jury is still out on these issues. There is clear evidence, as noted, of discrete, large-amplitude events or superpositions of a few waves which yield canonical spectral shapes in an Eulerian frame but which suggest a simpler Lagrangian viewpoint. Indeed, Hines [2001] stated "that the occurrence of the large-wave number Eulerian tail has nothing whatever to do with any physical process.... If one insists on defining 'waves' according to their Eulerian linear description, then one is forced to admit to the existence of 'nonlinear wavewave interactions'. . .but these are mere mathematical artifacts and have no physical import." However, while transformation to a Lagrangian coordinate does remove 


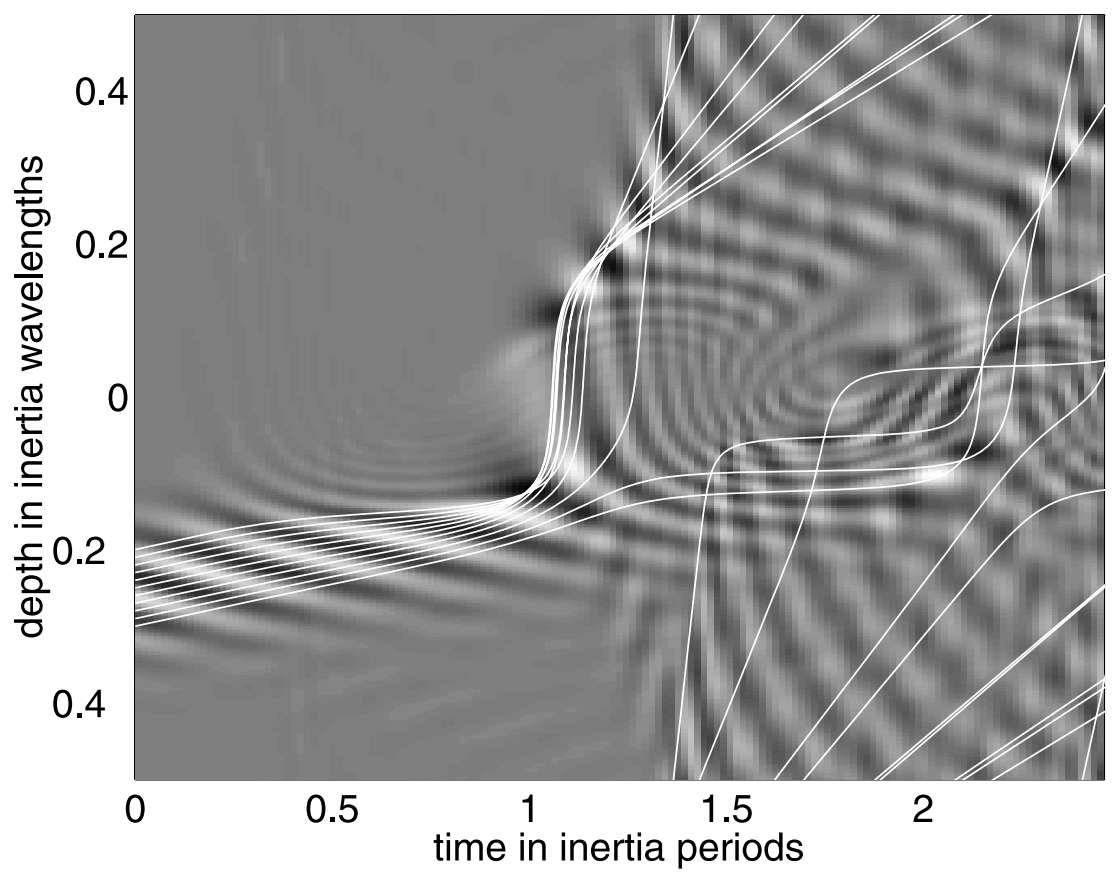

Figure 15. Perturbation density illustrating the encounter of a small-scale wave packet with an inertiagravity wave of much larger scale. In this case, the small-scale wave has a horizontal phase speed 0.2 of the maximum of the inertia-gravity wave motion, no critical level trapping occurs, and both the vertical wavelength and horizontal phase speed of the small-scale wave increase dramatically. After Broutman et al. [1997].

the advective nonlinearity in the Navier-Stokes equations, it does not remove the coupling of wave scales that nonlinearity implies. For example, claims of Lagrangian linearity cannot explain the nonlinear coupling of scales observed in nonlinear simulations or laboratory studies revealing significant wave-wave interactions [McEwan, 1971; Klostermeyer, 1991; Thorpe, 1994; Vanneste, 1995]. Likewise, shears due to mean winds and large-scale waves imply critical levels, small vertical scales, and instability processes that are demonstrably not mathematical artifacts. Indeed, Broutman et al. (personal communication, 2002) have pointed out that the dispersion relation employed by Hines [2001, 2002a] does not include the influences of mean wind shear on vertical wave structure and thus does not describe the full effects of Doppler shifting. The important issue is not whether nonlinear wave-wave (and wave-mean flow) interactions are important, as they surely are, but whether they are the dominant mechanism in constraining wave amplitudes and shaping the spectrum for $m<m_{M}$, as argued by Hines [2001] and Chunchuzov [2002], or whether they are one of several key processes acting in this fashion, which seems a more defensible perspective, given the various evidence available to date. The identified links between wave instabilities at small and large amplitudes [Sonmor and Klaassen, 1997], however, may render such distinctions obsolete.

[128] The Weinstock [1976, 1982, 1985] theory was discussed in previous reviews and has itself served as the basis for additional saturation theories by Zhu [1994] and Medvedev and Klaassen [1995] having various attributes. All of these theories view nonlinearity as spectral diffusion of one form or another. There is no clearly defined link, however, with the predictions of wave-wave interaction theory or direct numerical studies [e.g., Dunkerton, 1987; Klostermeyer, 1991; Vanneste, 1995], so testing of the physical basis for the theory has proved challenging. Recently, Hines [2002b] has raised serious objections to assumptions in the original work by Weinstock and its successors by association. However, while Weinstock's theory is clearly an approximation to a class of nonlinear effects, like Doppler spread theory, it calls attention to the importance of nonlinear interactions in shaping the wave spectrum with increasing altitude. Indeed, Hines's criticism has itself been recently challenged (Klaassen and Medvedev, personal communication, 2000). In summary, nonlinear wave-wave interactions must be regarded, at present, as a viable means of exchanging energy among gravity waves at various scales and frequencies throughout the middle atmosphere. Evidence suggests, however, that they cannot account, by themselves, for the general shape or amplitude of the gravity wave spectrum. Rather, they represent one of several nonlinear processes that act jointly to define gravity wave spectral character and evolution with altitude. Wave-wave interactions likewise do not contribute directly to energy dissipation, except through their links to specific instabilities at larger wave amplitudes [McEwan, 1971]. 


\subsection{Instability and Turbulence Dynamics}

[129] Theoretical, numerical, and observational studies have made substantial contributions to our understanding of instability and turbulence dynamics accompanying gravity waves in recent years. Perhaps the greatest advances were made possible by the continuing evolution of high-performance computers, which are now capable of direct numerical simulations (DNS) of stratified and sheared flows having resolutions of $\sim 1000^{3}$ and above. Such simulations are capable of describing both the transition to turbulence in a geophysical flow and the vorticity dynamics driving the turbulence cascade. Observational capabilities have also progressed significantly over the last decade or so and have yielded high-resolution in situ and ground-based measurements of wave and turbulence structures. Other numerical and laboratory studies have contributed to our understanding of general turbulence dynamics in applications not specific to atmospheric gravity waves.

[130] Turbulence is generally believed to arise locally within a gravity wave (or a field of superposed waves) when the flow is either convectively or dynamically unstable and the timescale for instability growth is sufficiently shorter than that describing the evolution of the wave field. More recent analyses have shown, however, that a wide spectrum of instabilities is possible, with specific instability character depending on wave amplitude and intrinsic frequency, mean shear and stability profiles, and the form and amplitude of perturbations triggering flow instability. In particular, local instability can occur at wave amplitudes below that often considered necessary for convective instability in many instances [McEwan, 1971; Hines, 1988b; Lombard and Riley, 1996; Sonmor and Klaassen, 1997]. It is also believed, based on simple stability arguments, that dynamical instability should predominate for intrinsic frequencies $\hat{\omega} \sim f$ and that convective instabilities should predominate for intrinsic frequencies $\hat{\omega} \gg f[$ Dunkerton, 1984; Fritts and Rastogi, 1985] because of the very different relative amplitude thresholds in each case. These expectations are supported by the numerical simulations performed to date [Andreassen et al., 1994, 1998; Fritts et al., 1994, 1998, 2003; LeLong and Dunkerton, 1998a, 1998b].

[131] Because local instabilities at wave amplitudes below that normally identified as the "convective" instability limit,

$$
a=u^{\prime} /(c-\bar{u})=1.0,
$$

generally exhibit slow growth, however [Lombard and Riley, 1996; C. Bizon, personal communication, 2001], we assume for our discussion that the nominal threshold amplitudes for dynamical and convective instability are those which yield $R i<1 / 4$ and $R i<0$, respectively, with the Richardson number $(R i)$ defined to be

$$
R i=\frac{N^{2}}{\bar{u}_{z}^{2}+\bar{v}_{z}^{2}},
$$

where $\bar{u}_{z}$ and $\bar{v}_{z}$ are the local mean zonal and meridional wind shears and $N$ is the local buoyancy frequency. For most intrinsic frequencies the two threshold amplitudes are nearly identical [Dunkerton, 1984; Fritts and Rastogi, 1985], departing significantly only for $\hat{\omega} \sim f$.

[132] With the high-resolution simulations now possible on current supercomputers, DNS studies of gravity wave breaking at sufficiently high intrinsic frequencies (i.e., more nearly comparable, rather than disparate, horizontal and vertical wavelengths) can capture both gravity wave scales and a broad range of turbulence scales simultaneously. On the other hand, the large disparity between inertia-gravity wave and turbulence scales $\left(\sim 10^{6}\right.$ or more) prevents simulations that span this range of scales. Instead, relevant simulations have addressed the $\mathrm{KH}$ instability of inertia-gravity waves and the turbulence arising from $\mathrm{KH}$ instability separately.

\subsubsection{Gravity Wave Breaking}

[133] A number of numerical studies addressed gravity wave breaking and the accompanying wave amplitude limits in 2-D for lack of adequate computational resources to perform full 3-D studies. Because the instability processes are inherently 3-D, however, 2-D studies are either limited in their utility (e.g., in quantifying wave amplitude limits and momentum flux divergence) or complete misrepresentations of the relevant dynamics (e.g., in modeling wave breaking and turbulence dynamics) [Andreassen et al., 1994]. Thus, we will review here only those studies addressing the 3-D character of wave instability or the occurrence and scales of such events in the atmosphere.

[134] The first 3-D numerical studies of gravity wave instability dynamics in the atmosphere were performed by Andreassen et al. [1994], Fritts et al. [1994], and Isler et al. [1994]. A parallel study examining similar dynamics in an oceanic context was performed by Winters and D'Asaro [1994]. These studies addressed gravity wave breaking via convective instability, which appears to be the preferred instability for gravity waves at relatively high intrinsic frequencies [Dunkerton, 1984; Fritts and Rastogi, 1985]. These simulations succeeded in describing the character of the primary wave instability process; this comprises counterrotating streamwise (along the flow) convective rolls or vortices (with spanwise, or normal, wave number). These convective rolls occupy the full depth of the convectively unstable region within the gravity wave, derive their eddy energy (or vorticity) from baroclinic and shear sources within the 2-D flow, and trigger a turbulence cascade via mutual vortex interactions as they interact with adjacent shear layers (or vortex sheets). Indeed, the initial study of gravity wave breaking in 3-D provided a plausible explanation for apparent streamwise instability structures observed in NLC near the summer mesopause [Fritts et al., 1993b] (see Figure 16). Additional observational evidence of such instabilities has come from recent analyses of air- 

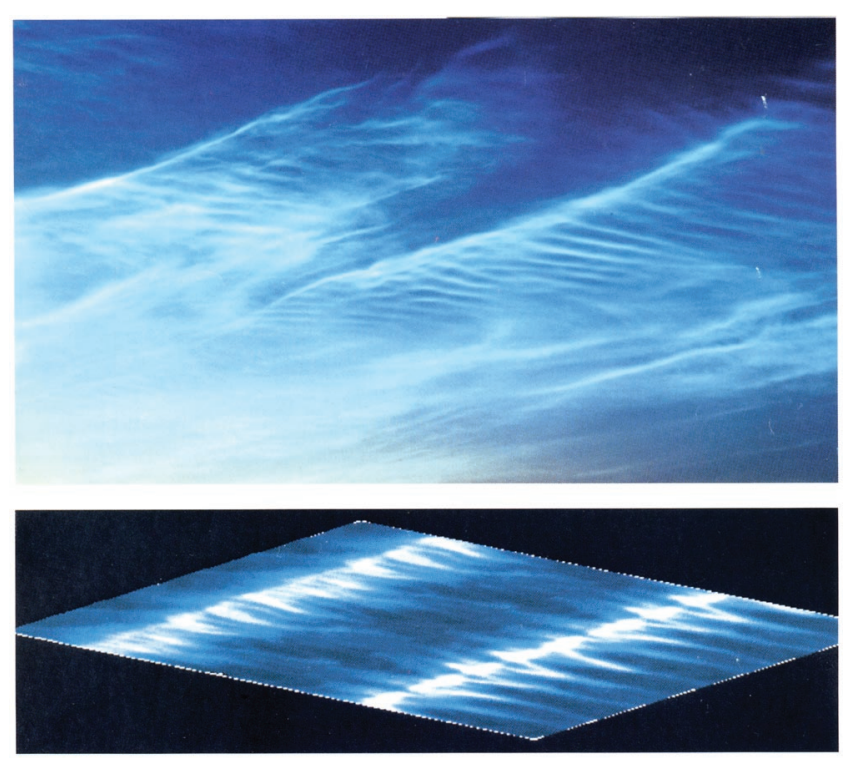

Figure 16. (top) Observed and (bottom) simulated gravity wave and instability structures in NLC. Photo was taken at Turku, Finland, on 21-22 July 1989, courtesy of Pekka Parvianien. Streamwise-aligned instability structures are believed to have accounted for the smaller-scale bright bands oriented approximately normal to the gravity wave phase fronts in the upper image. After Fritts et al. [1993b].

glow imaging data and correlative wind measurements [Swenson and Mende, 1994; Hecht et al., 1997, 2000].

[135] Subsequent numerical studies at higher resolution addressed wave breaking in streamwise and transverse shear flows [Fritts et al., 1996a], with superposed low- and high-frequency motions [Fritts et al., 1997b], and the vorticity dynamics driving the turbulence cascade [Andreassen et al., 1998; Fritts et al., 1998]. The latter studies addressed in detail the vorticity dynamics of the initial shear-aligned instabilities as well as their subsequent interactions with adjacent vorticity sheets (the mean and 2-D gravity wave shears having spanwise vorticity).

[136] The vorticity field arising because of convective instability of the gravity wave, the subsequent transition to turbulence, and the dynamics within the turbulent flow are illustrated in Figure 17 at various times throughout the simulation described by Andreassen et al. [1998] and Fritts et al. [1998, 1999]. The simulation was performed for a gravity wave of horizontal wavelength $\sim 30$ $\mathrm{km}$ and intrinsic frequency at the level of wave breaking of $\sim N / 6$. A mean shear was imposed to confine the breaking to the region below the initial critical level for the wave. The entire transition from a 2-D overturning gravity wave to quasi-isotropic turbulence occupies approximately a buoyancy period in this simulation. Vortex structures are displayed using a quantity representing the rotational or "tube-like" character of the motion field analogous to the minimum pressures within the flow (see Jeong and Hussain [1995] and Andreassen et al.
[1998] for further details). The volumes are viewed from below, with the streamwise direction and wave propagation to the right.

[137] The transition from 2-D laminar flow to 3-D quasi-isotropic turbulence accompanying wave breaking involves several distinct phases: (1) the initial shearaligned convective instability within the gravity wave (first two images in Figure 17), (2) a second phase in which divergent spanwise motions below adjacent streamwise vortices stretch and thin the adjacent spanwise vortex sheets, causing them to become locally dynamically unstable, (3) the formation of secondary, spanwise-localized KH billows on the intensified vortex sheets and their linkage to the overlying streamwise vortices to form series of intertwined vortex loops (second, third, and fourth images in Figure 17), and (4) the subsequent interactions of neighboring vortices, the excitation of twist waves on the various vortices, and the unraveling, fragmentation, and breakup of the vortices that comprises the cascade to smaller scales of motion (last four images in Figure 17). Indeed, the various stages in the transition to turbulence often occur nearly simultaneously in different (or the same) portions of the flow. Similar simulations having a spanwise mean shear component and more recent higher-resolution simulations having no mean shear confirm the general nature of the transition and turbulence dynamics described above [Fritts et al., 1996a, 2003].

[138] Various stages in the turbulence evolution noted above also parallel in important respects vortex dynamics observed in other turbulent flows. Intertwined vortex loops, or similar "hairpin" or "horseshoe" vortices, are seen to arise in sheared boundary layers [Gerz et al., 1994; Adrian et al., 2000]; twist waves, so easily excited in the wave breaking simulations, were also observed, but not recognized as such, in laboratory studies of vortex dynamics [Cadot et al., 1995]. Finally, the sequence of vortex dynamics seen in gravity wave breaking exhibits striking parallels to that observed in the transition to turbulence accompanying the $\mathrm{KH}$ instability to be discussed below [see also Arendt et al., 1997, 1998; Fritts et al., 1999]).

[139] The scales at which gravity wave breaking occurs are becoming better known based on both direct and indirect measurements. Such observations also address the current debate over the mechanisms constraining wave amplitudes and imposing saturation of the gravity wave spectrum (see sections 6.1 and 6.3). Direct measurements of density or temperature (via lidar and balloon- or rocket-borne instrumentation) reveal frequent occurrences of near-adiabatic or superadiabatic lapse rates, with occurrence frequency and vertical scale increasing with altitude. Recent examples include balloon and Rayleigh lidar measurements of large-amplitude wave motions at lower altitudes [Hauchecorne et al., 1987; Shutts et al., 1988; Wilson et al., 1991a; Whiteway and Carswell, 1995; Hoppe et al., 1999] and sodium resonance lidar measurements at greater altitudes 

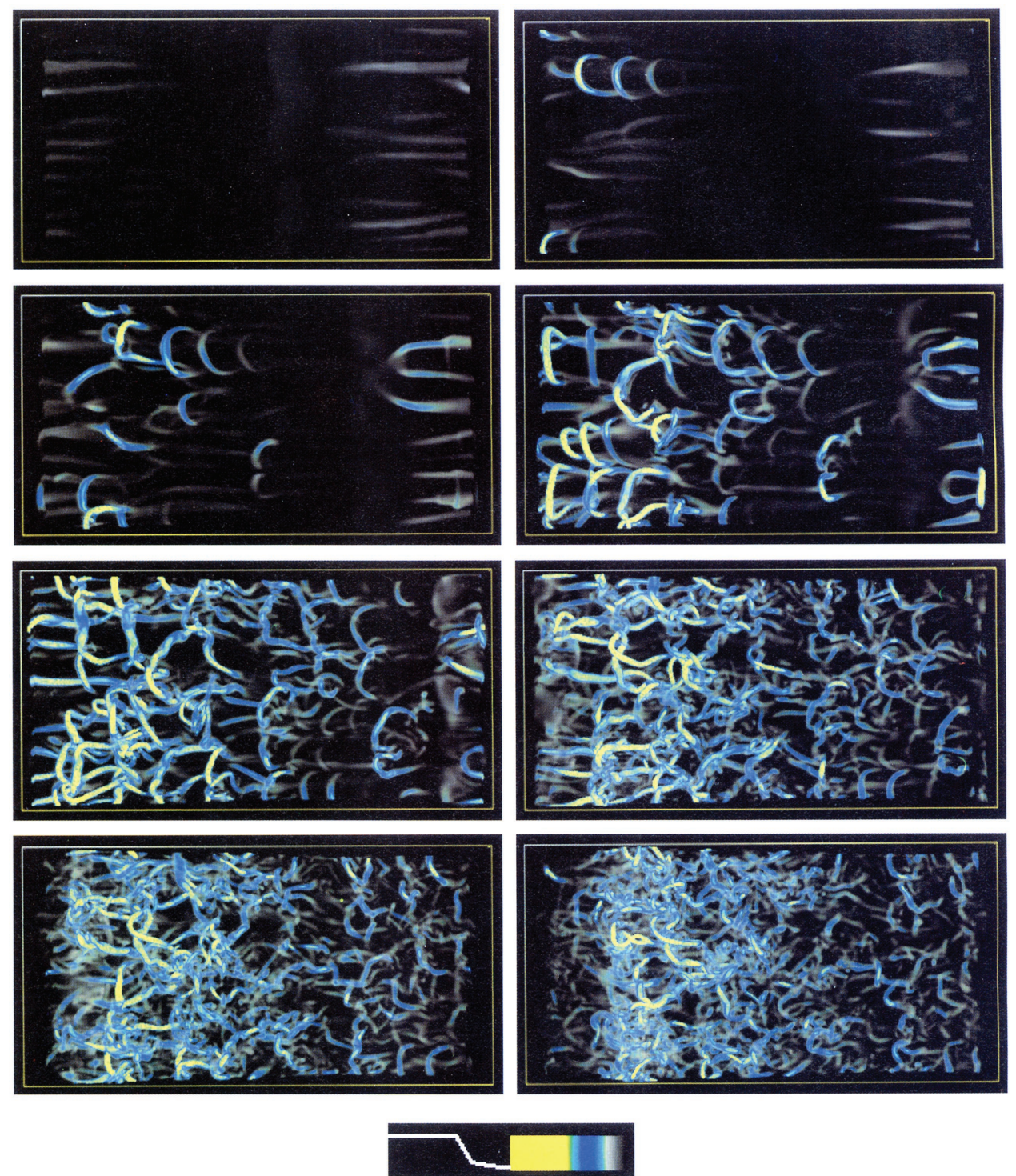

Figure 17. Vorticity structures in a breaking gravity wave viewed from below with the direction of wave propagation to the right. The images display vortex structures from left to right and top to bottom, spanning one buoyancy period following attainment of convective instability of the wave field. See text for details. After Fritts et al. [1999] (reprinted with permission of Terra Scientific Publishing Company).

[Hecht et al., 1997; Williams et al., 2002]. Rocket measurements employ various techniques, including falling spheres [Fritts et al., 1988b], chaff [Wu and Widdel, 1991], ionization gauges [Lübken, 1997], and Rayleigh lidars [Hoppe et al., 1999]. These observations reveal unstable or nearly unstable layers typically up to a few kilometers in depth, suggesting convectively unstable gravity waves having vertical wavelengths of $\sim 3-10 \mathrm{~km}$ or more, with the larger scales more prevalent near the mesopause. The recent sodium lidar measurements by Williams et al. [2002] represent a particularly striking example, with multiple superadiabatic layers a few kilometers in depth within a near-adiabatic layer extending more than $10 \mathrm{~km}$ and persisting for more than 4 hours (see Figure 18). As noted above, these observations suggest, at least in this instance, wave saturation via local convective instability, though wave superposition or wave-wave interactions, specifically the PSI displayed in Figure 13, may play a role in the vertical fine structure within the overturning wave field. While this event is exceptional, the frequency of observation of such features suggests that they are far from the infrequent or pathological cases implied by Hines [1996]. Indirect inferences of convective overturning scales are provided by NLC and airglow measurements of horizontal instability scales [Fritts et al., 1993b; Swenson and Mende, 1994; Hecht et al., 1997, 2000], where the observed roll spacing is suggested to be comparable to the unstable layer depth in numerical simulations. Additional inferences of gravity wave amplitudes and instability depths are obtained from radar wind measurements via the gravity wave dispersion relation [Muraoka et al., 1988]. 
a. Temperature $(K)$

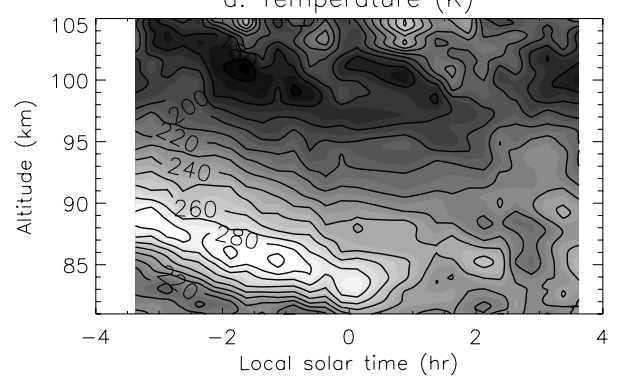

c. $\%$ variation of $\mathrm{T}$
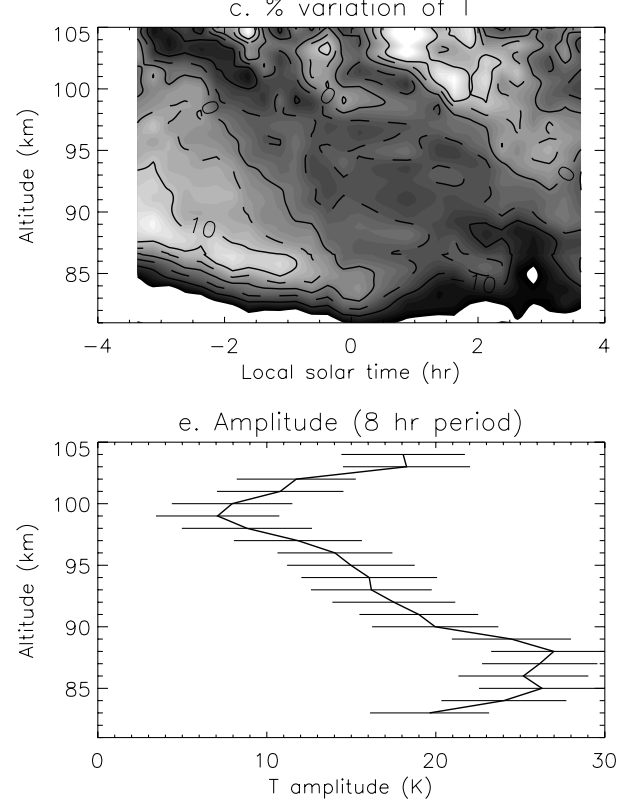

b. Sodium Density $\left(\mathrm{cm}^{-3}\right)$

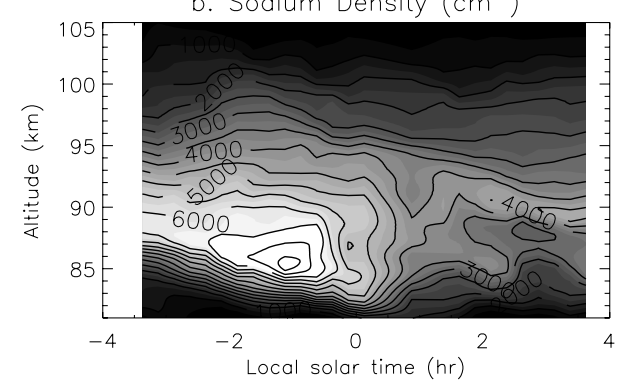

d. Mean temperature
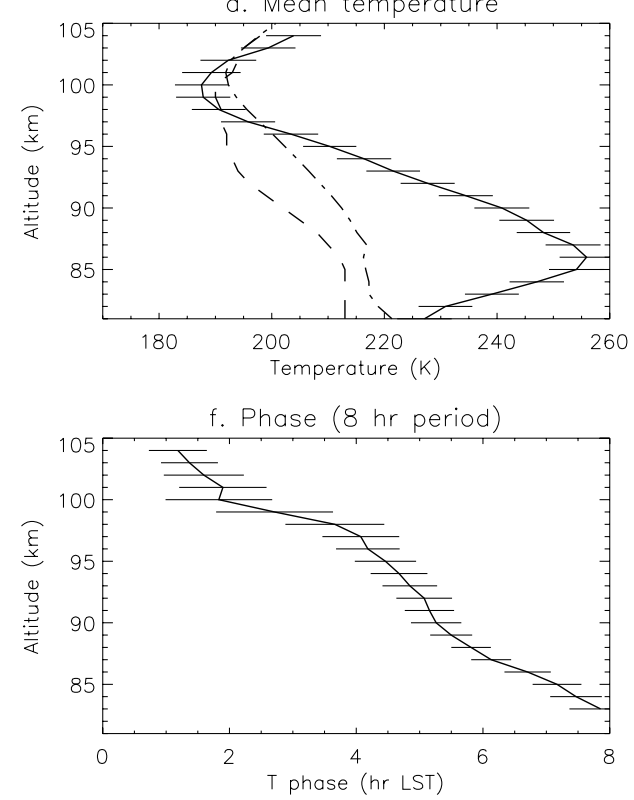

Figure 18. Contours of (a) temperature, (b) sodium density, (c) temperature perturbations as a percent of the mean value, and (d) profile of mean temperature for the 8-hour wave observation using the Fort Collins sodium lidar on day 307 of 1997 . Also shown are the amplitude of the 8-hour wave in the temperature field (e) and the phase of the maximum temperature (f). The $1 \sigma$ uncertainties are shown with horizontal bars and the dashed and dash-dotted lines in Figure 18d are the diurnal means for a 24-hour campaign in November 1998 and nightly mean for November averaged from 1990 to 1997. After Williams et al. [2002].

\subsubsection{Kelvin-Helmholtz Instability}

[140] KH instability is among the most common sources of turbulence in the atmosphere. In many instances it owes its existence, in part at least, to wind shears due to inertia-gravity waves because such motions contribute preferentially to wind shears throughout the atmosphere. Earlier evidence of $\mathrm{KH}$ instability due to inertia-gravity waves was reviewed by Fritts and Rastogi [1985]. More recent stability analyses have identified the preferred modes of instability of inertia-gravity waves with and without mean shears [Fritts and Yuan, 1989c; Yuan and Fritts, 1989; Dunkerton, 1997a]. Numerical simulations of unstable inertia-gravity waves revealed the character of the $\mathrm{KH}$ instability and its preferred orientation within the wave field [LeLong and Dunkerton, 1998a, 1998b]. The numerical studies showed a preferred direction consistent with the stability analysis by Fritts and Yuan [1989c] but with an increasing degree of isotropy as $\hat{\omega} \rightarrow f$. These simulations were also used to assess the theoretical prediction of the wave amplitude required for onset of shear instability by Dunkerton [1984] and Fritts and Rastogi [1985]. Results are displayed in Figure 19 and reveal that time dependence of the wave field increases the wave amplitude required to initiate instability, as anticipated by Lombard and Riley [1996] and Sutherland [2001] at higher intrinsic frequencies.

[141] Other evidence of the importance of $\mathrm{KH}$ instability and its relation to inertia-gravity waves comes from observations of wind and temperature profiles in the lower and middle stratosphere. Radar measurements at several sites have revealed persistent layers of enhanced radar reflectivity with spacings of a few kilometers, often exhibiting slow vertical motions and inferred unstable wave amplitudes [Sato and Woodman, 1982; Yamamoto et al., 1987]. Likewise, high-resolution balloon measurements have revealed multiple layers yielding signatures of local turbulent mixing, with near-adiabatic layers sandwiched between sharp temperature inversions [Cot and Barat, 1986; Coulman et al., 1995]. Examples of the 


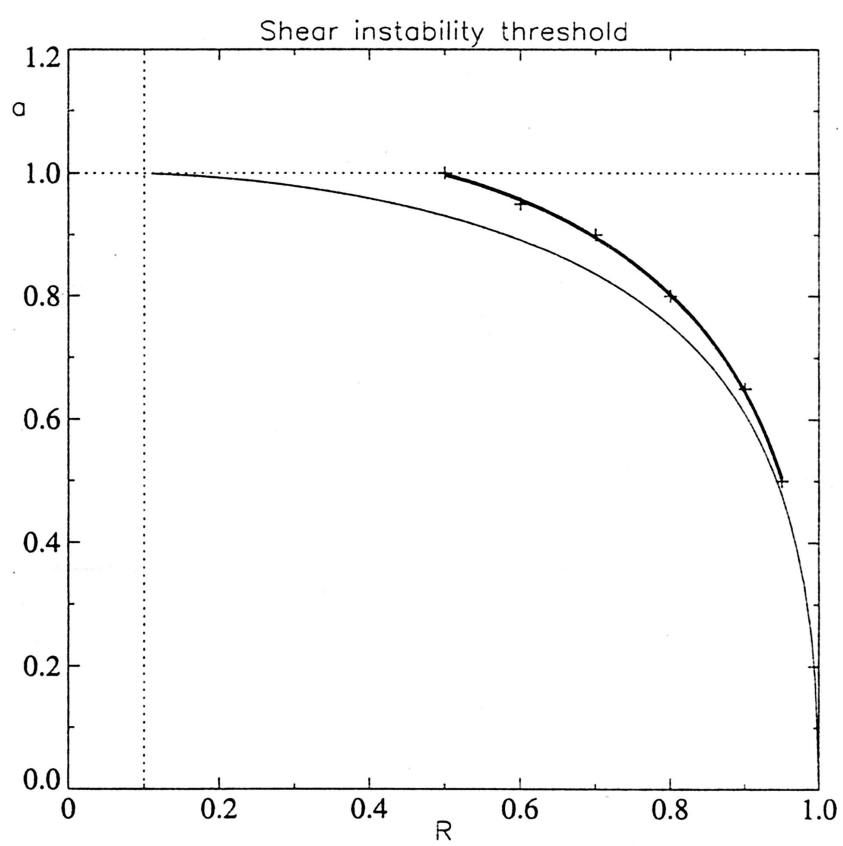

Figure 19. Threshold amplitude $a$ for shear (KH) instability of inertia-gravity waves as a function of $R=f / \hat{\omega}$ in the absence of mean shear based on theory for a time-independent flow (solid line) and DNS simulations (thick solid line with data points). After LeLong and Dunkerton [1998a] (reprinted with permission of the American Meteorological Society).

latter, with profiles of the corresponding Richardson number, are displayed in the top and middle panels of Figure 20. Note, in particular, that the observations suggest a minimum Richardson number of $\sim 1 / 4$ following instability and mixing, well below what was often assumed to accompany restratification in the past. It will be seen below that these measurements are in good agreement with recent high-resolution DNS studies of $\mathrm{KH}$ instability.

[142] While observations help define the scales at which $\mathrm{KH}$ instability and mixing occur and the consequences of mixing for the temperature and wind fields, they cannot identify the dynamics of the turbulence transition or the evolution of the shear layer as $\mathrm{KH}$ breakdown and mixing occur. The only means of understanding these aspects of inertia-gravity wave instability is via DNS studies spanning $\mathrm{KH}$ growth, breakdown, and restratification.

[143] There have been many numerical studies of KH instability in the past. Only recently, however, have computational capabilities permitted 3-D studies of high enough resolution to confirm the predictions and laboratory observations of secondary instability [Klaassen and Peltier, 1985; Thorpe, 1987; Palmer et al., 1994, 1996; Caulfield and Peltier, 1994; Fritts et al., 1996b; Smyth, 1999]. Early 3-D KH simulations were not adequate to describe the transition to turbulence, the structure and anisotropy within the inertial-range of turbulence, and the implications for dissipation and mixing. However, recent simulations are now adequate to investigate these turbulence effects [Werne and Fritts, 1999, 2001; Smyth and Moum, 2001]. The vorticity dynamics accompanying this evolution and its relation to the vorticity dynamics of wave breaking were described by Fritts and Werne [2000].

[144] The KH simulations performed by Werne and Fritts [1999, 2001] have illustrated the dynamics of the transition to turbulence, the expansion of turbulence throughout the KH billow and the shear layer, and the turbulence statistics, dissipation, anisotropy, and decay. Most striking, perhaps, is the great degree of similarity of the turbulence dynamics to those observed in previous simulations of gravity wave breaking. Common features include (1) the initial streamwise, or shear-aligned, convective instability within the outer regions of the $\mathrm{KH}$ billow, (2) the stretching and wrapping of spanwise vortex sheets around the streamwise vortices, (3) the dynamical instability and rollup of the localized and intensified vortex sheets, and (4) the subsequent vortex interactions and twist wave excitation driving the cascade to smaller scales of motion [Fritts and Werne, 2000]. Major differences in the turbulence transitions accompanying wave breaking and shear instability are the timescale and the character of mixing. As noted above, the transition due to high-frequency wave breaking requires approximately one buoyancy period for the wave parameters simulated. The transition is somewhat longer due to $\mathrm{KH}$ instability, however, because turbulence requires time to expand from the initial site of instability throughout the KH billow and the full depth of the evolving shear layer. Implications for mixing are probably very different as well since $\mathrm{KH}$ instability mixes the shear layer vigorously prior to restratification, whereas turbulence due to wave breaking is quickly advected out of the unstable phase of the wave motion, allowing vigorous turbulence to act within the stably stratified portions of the wave field.

[145] The vorticity dynamics and impact on the thermal field of KH turbulence are illustrated in Figure 21 at four times throughout the simulation described by Werne and Fritts [1999]. In this simulation, a buoyancy period corresponds to 28 time units. Note here the gradual penetration of turbulence throughout the KH billow, the rapid obliteration of thermal gradients by turbulence shortly after it occurs, the homogenization of the expanded shear layer, the very sharp thermal gradients that evolve as a result of efficient mixing within the shear layer, and the continuing small-scale dynamics and mixing within the edge regions of the mixed layer at later times. Profiles obtained in the KH DNS at $t=165$ are displayed in the fourth panel of Figure 20 and exhibit good agreement with those observed by Coulman et al. [1995] (first and second panels), despite the markedly different Reynolds numbers of the two flows. The implications for inertia-gravity waves include (1) instability, turbulence, and mixing on timescales short compared to wave periods, hence confined to the unstable phase of the wave, (2) reduced shears and wave amplitudes due to mixing, and (3) alternating layers of low and high static 

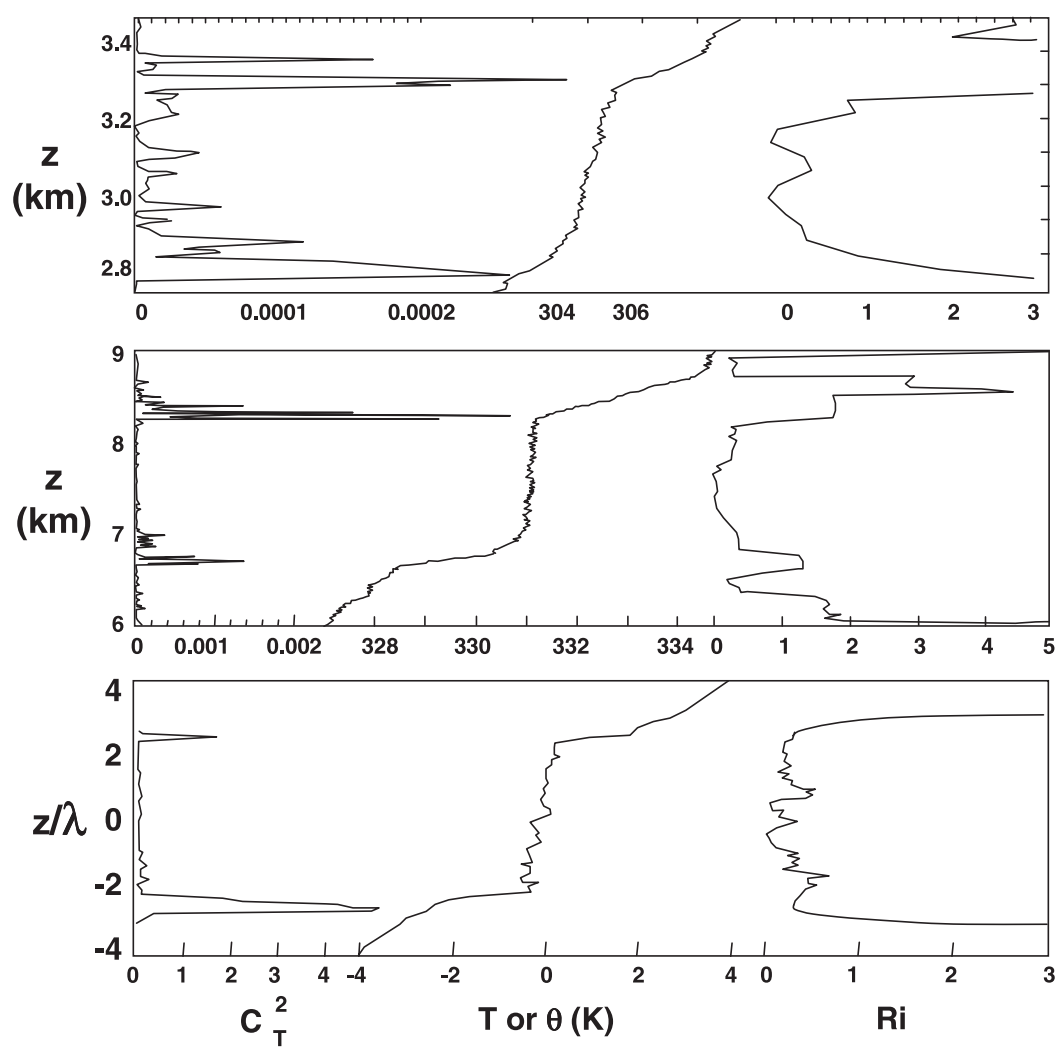

Figure 20. Profiles of $C_{T}^{2}, \theta$, and $R i$ obtained by (top and middle) high-resolution balloon measurements and (bottom) DNS showing the aftermath of $\mathrm{KH}$ instability and mixing events. Note that the magnitude of $\mathrm{C}_{T}^{2}$ and the gradient of $\theta$ maximize at the edges of the mixed region and that $R i \approx 1 / 4$ throughout the mixed region. Modified after Coulman et al. [1995] and Fritts et al. [2003].

stability that may influence subsequent instability and mixing processes.

\subsubsection{Synthesis and Other Instability and Large- Amplitude Processes}

[146] Studies cited above have expanded greatly our understanding of gravity wave instability processes over the last decade. Importantly, these studies have also suggested links between specific modes of instability of small- and large-amplitude gravity waves. Klostermeyer [1991] found the PSI to be related to a 2-D parametric instability at finite amplitude and suggested, based on his analysis, that resonance may underlie all gravity wave instability processes. Klostermeyer [1991] and Lombard and Riley [1996] also identified transverse modes of instability at finite amplitudes below those required for local convective or dynamical instability.

[147] The links between instabilities at small and large wave amplitudes were further clarified and generalized by Sonmor and Klaassen [1997] to include all instability types and all wave amplitudes and intrinsic frequencies (or phase propagation angles) assuming no rotation. Specifically, they found a link between the PSI and the "slantwise static instability" (SSI) of Hines [1971, 1988b] at small wave amplitudes and with convective instability and high wave number parametric instability at amplitudes $a>1$ (see equation (58)). Sonmor and Klaassen
[1997] further linked the "branch-C" instability of Yeh and Liu [1981] to the resonant elastic scattering and induced diffusion interactions originally identified by McComas and Bretherton [1977] but noted quite different growth rates than inferred in the earlier study. The "shear-aligned instability" (SA, having spanwise wave number, as noted in the wave breaking simulations discussed in section 6.2.1) is itself linked to higher-order resonant instabilities at smaller wave amplitudes and exhibits an amplitude threshold near the convective limit for waves at high intrinsic frequencies [Winters and Riley, 1992; Sonmor and Klaassen, 1997]. A 2-D dual-mode instability represents a generalization of the $\mathrm{KH}$ instability within an inertia-gravity wave and competes favorably with SA or convective instability at large amplitudes [Sonmor and Klaassen, 1997] but is itself stabilized by weak environmental shear [Thorpe, 1994]. Dunkerton [1997a] examined the relative roles of these instabilities in the presence of rotation, employing a steady, planeparallel approximation to the wave structure and obtained similar results concerning the dominant instabilities at large wave amplitudes.

[148] A further class of instability, "oblique instabilities" (having nonzero streamwise and spanwise wave numbers), was found by Lombard and Riley [1996] and Sonmor and Klaassen [1996, 1997] to represent the most rapidly growing instability for an important physical 

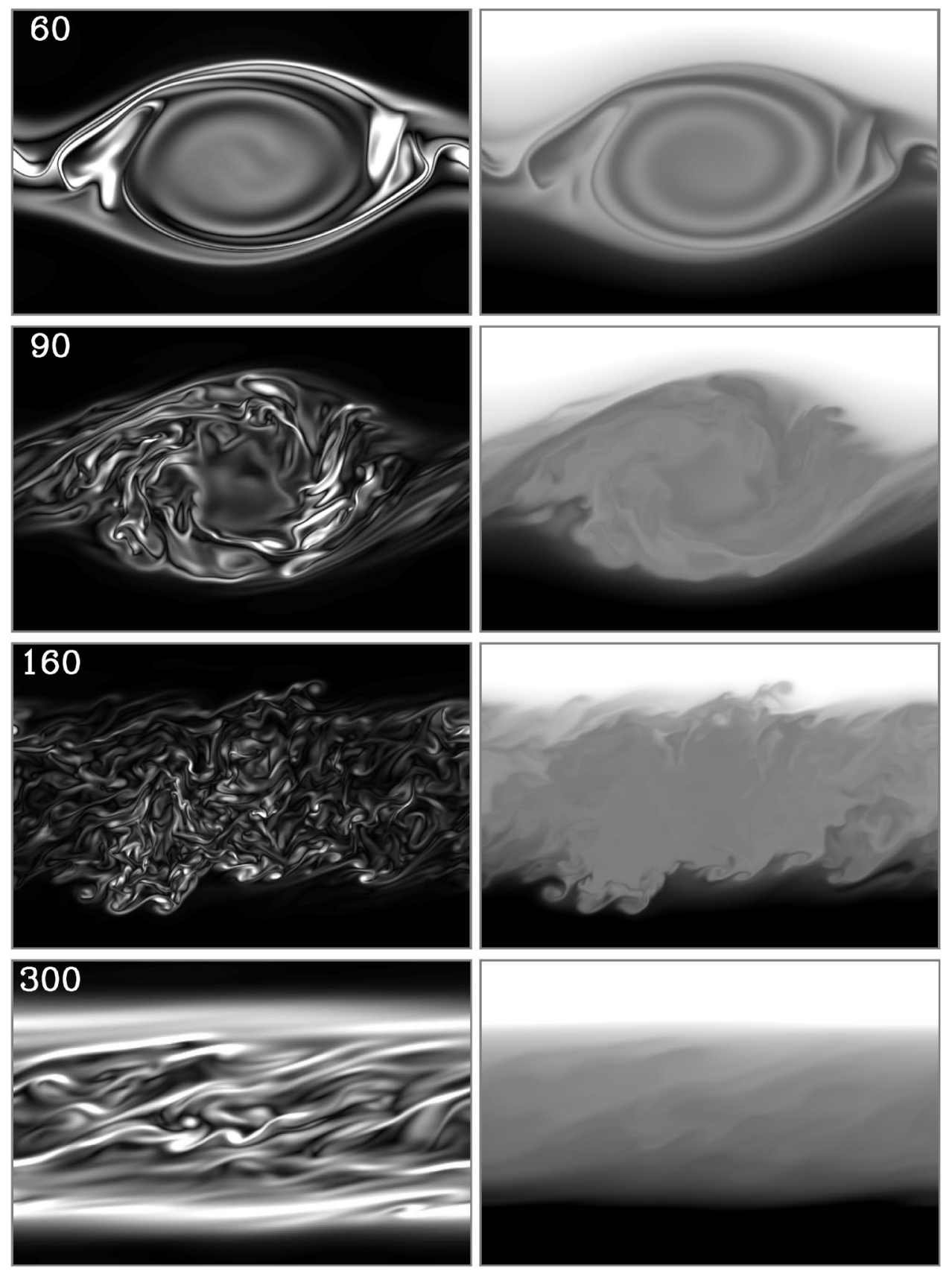

Figure 21. The 2-D streamwise-vertical cross sections of (left) 3-D vorticity magnitude and (right) temperature fields at four times throughout the turbulence transition and restratification of a KH billow. See text for details. After Werne and Fritts [2001] (reprinted with permission of Elsevier Science).

range of wave amplitudes at high intrinsic frequencies. Sonmor and Klaassen [1996, 1997] argue that oblique instabilities connect smoothly to SA instabilities at higher frequencies and larger wave amplitudes but are the preferred form when shear is the primary source of instability energy, whereas SA instabilities are preferred when buoyancy is the dominant source. The parameter ranges in which these various instabilities predominate are displayed in Figure 22. Here the dash-dotted line denotes an overturning amplitude, and high intrinsic frequencies have large phase elevation angles. We note, however, that recent DNS studies [Fritts et al., 2003] reveal SA instabilities to predominate over 2-D dualmode and oblique instabilities at finite amplitude for a wide range of the wave parameters displayed in Figure 22. This may be due either to the Sonmor and Klaassen analysis being inviscid (while the DNS studies are viscous) or to the selection of a finite-amplitude response in the DNS from among a variety of initial instabilities at infinitesimal amplitude.

[149] Other types of instabilities are enabled as a result of wave packet localization or by the induced mean flows accompanying waves of finite amplitude. Sutherland [2001] demonstrated numerically the modulational 


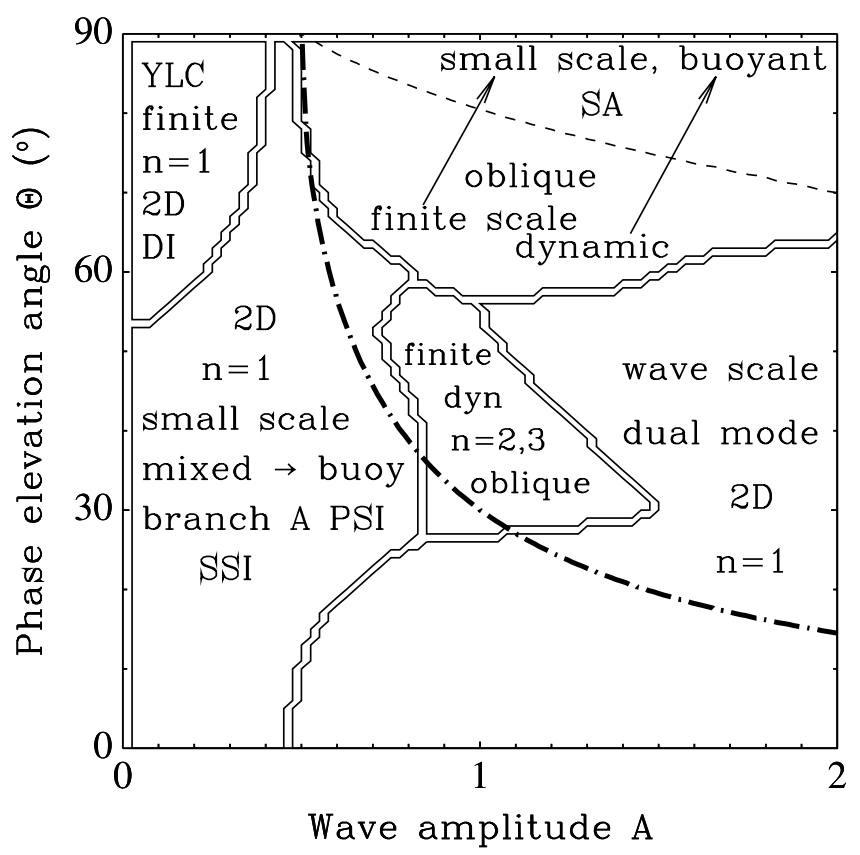

Figure 22. Fastest growing linear instabilities as a function of wave amplitude and phase elevation angle. The dash-dotted line corresponds to the amplitude of overturning ( $a=1$ in our terminology), and the dashed line represents the transition from oblique to shear-aligned instability and from shear to buoyancy as the dominant source of eddy energy. After Sonmor and Klaassen [1997] (reprinted with permission of the American Meteorological Society).

instability (and stability) of a localized wave packet anticipated by Whitham [1965, 1974] but noted that this instability does not necessarily imply wave breaking. Sutherland also argued that self-acceleration of a vertically localized wave packet can lead to local convective instability at sufficiently large amplitudes and high intrinsic frequencies, providing a means by which wave localization can contribute to instability. The mechanism by which instability occurs is the differential tilting of surfaces of potential temperature by the wave-induced mean flow. Similar effects accompany localized wave packets incident on a turning level and may lead to wave instability via either self-acceleration (as above) or as a result of enhanced wave amplitude due to incident and reflected wave superposition [Sutherland, 2000]. Interesting additional consequences of wave packet localization and finite amplitude include tendencies (1) for wave packets that are vertically localized and of large amplitude to yield permanent momentum flux divergence near a turning level and (2) for wave packets that are both horizontally and vertically localized to penetrate substantial distances beyond a turning level based on linear theory [Sutherland, 1999, 2000]. Indeed, both of these mechanisms may have important but as yet unquantified implications for gravity wave momentum transport and redistribution in the middle atmosphere. The tendency for spatially localized, large-amplitude packets to penetrate turning levels may be particularly
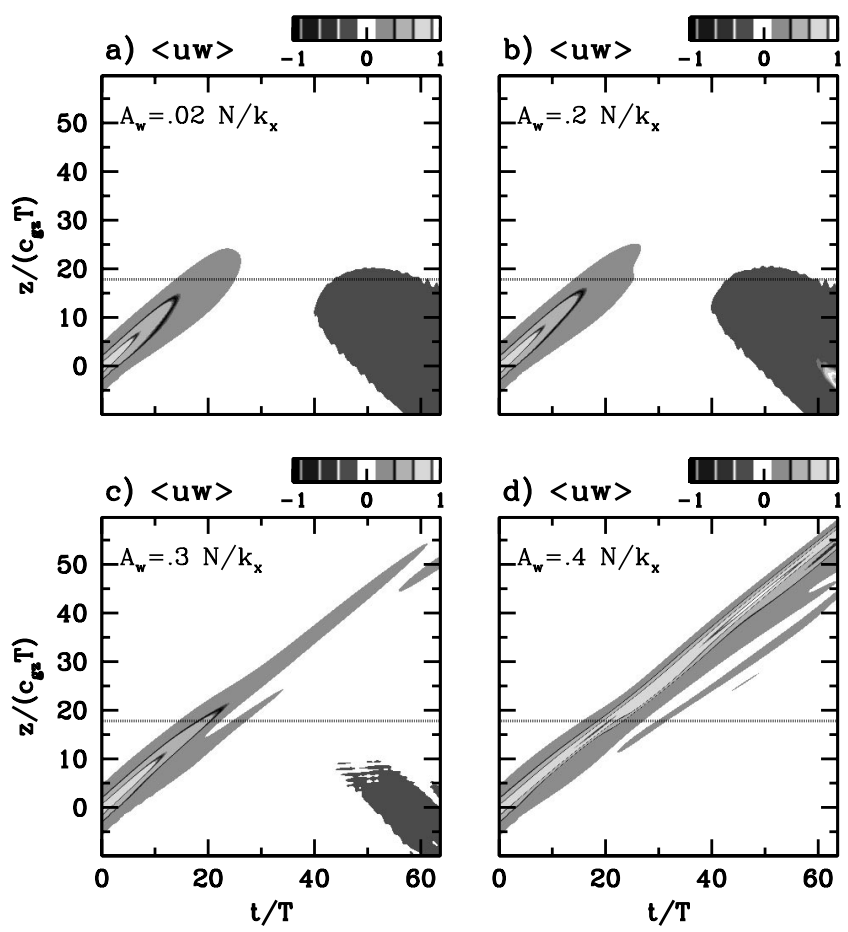

Figure 23. Time series of wave momentum flux for wave amplitudes of various vertical velocities incident on a turning level. The turning level anticipated by linear theory is shown by the horizontal line in each panel. After Sutherland [2000] (reprinted with permission of the Royal Meteorological Society).

relevant for middle atmosphere momentum transport, where such packets may be more the rule than the exception. A demonstration of the tendency for penetration of a turning level as wave amplitude increases is shown in Figure 23. In these cases, total and partial reflection occur for small and intermediate wave amplitudes, while wave packet propagation appears to be increasingly unaffected by mean shear (and increasing intrinsic phase speed and frequency) as wave amplitude increases. Wave packet transmission and reflection for the largest wave amplitude are displayed at several stages throughout the event in Figure 24. Note, in particular, the phase distortions at the leading edge of the wave packet above the turning level which exhibit the self-acceleration effects discussed by Fritts and Dunkerton [1984] and Sutherland [1999, 2000].

[150] A final topic relevant to our understanding of instability dynamics in gravity waves is the optimal perturbation theory pioneered by Farrell and Ioannou [1996a, and references therein]. This theory has provided considerable insights into the occurrence of or competition among various instability modes in Couette, Poiseuille, and other time-independent shear flows known to be asymptotically stable below a threshold (or for any) Reynolds number. However, the theory also can be applied to time-dependent flows and to more general stratified problems including secondary $\mathrm{KH}$ and gravity wave instabilities for which the equations of motion are 

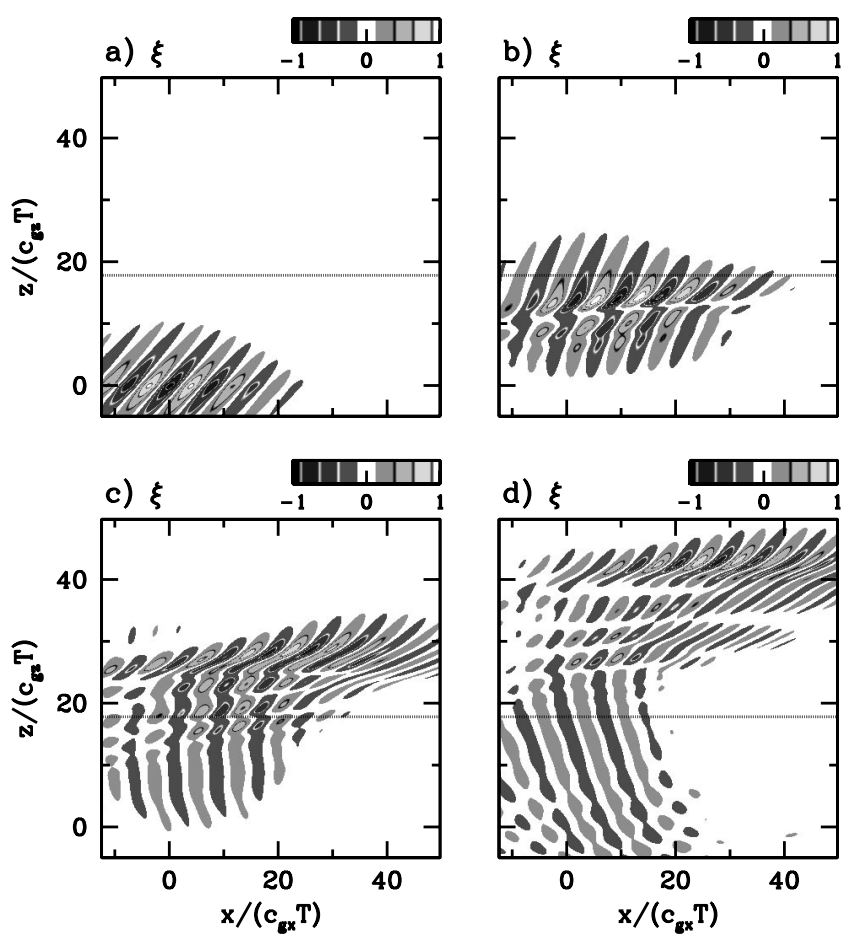

Figure 24. As in Figure 23 but showing vertical displacements at four times throughout the turning level encounter for the wave packet of largest amplitude. After Sutherland [2000] (reprinted with permission of the Royal Meteorological Society).

non-self-adjoint [Farrell and Ioannou, 1996b]. In such flows, particular superpositions of linear modes, termed "optimal perturbations", may exhibit rapid transient growth exceeding the growth rates of all eigenfunctions of the linear system. If this growth achieves a sufficient amplitude to have nonlinear consequences, either among the perturbations or for the mean flow, then it will be the optimal perturbation which dictates the finite-amplitude behavior of the nonlinear system. In applications to the $\mathrm{KH}$ instability problem, the optimal perturbation methodology has shown that the structures of both the initial 2-D instability and the secondary 3-D instabilities depend sensitively on the form and amplitude of the initial perturbations (J. Werne, personal communication, 2001). Optimal perturbation theory also reveals why the details of the turbulence transition and cascade discussed above depend so sensitively on the initial noise spectrum [Werne and Fritts, 1999; Fritts and Werne, 2000]. In the atmosphere, then, we anticipate that instability structures accompanying wave breaking or shear instability may often be dictated more by other finite amplitude perturbations than by the exact form of the most rapidly growing linear eigenmode of the unstable flow. While initial perturbations dictate the details of the transition to turbulence, they appear not to alter the statistical effects of turbulence on the larger-scale flow [Fritts and Werne, 2000].

\subsection{Saturation Theories}

[151] The dynamics underlying gravity wave saturation in the atmosphere, i.e., instability and wave-wave interaction processes, appear to impose a near-universal spectral form far from the dominant sources at lower altitudes (but subject to all of the caveats about departures from universality noted above). Our purpose in this section is to review the various theories for the shape and evolution of the wave spectrum and to identify how they are linked to the instability dynamics discussed above.

[152] The earliest wave amplitude limits attributed to instability dynamics were proposed by Hodges [1967] and involved the convective instability of a gravity wave due to its exponential growth with height. Subsequent studies by many authors considered the implications of such dissipation for momentum transports, turbulence, and mixing (see Fritts [1984a] for a review). Motivated by VanZandt's [1982] suggestion of a universal gravity wave spectrum and their own observations of near universality in stratospheric wind spectra [Dewan et al., 1984], Dewan and Good [1986] proposed the first theory of a saturated spectrum employing saturation (via local convective or shear instability) separately at each vertical wave number. Their dimensional analysis yielded a saturated range for gravity waves varying as $C N^{2} / m^{3}$ with $C$ dependent on spectral bandwidth (and a bandwidth $d m \sim m$ ). The study by Smith et al. [1987] extended this theory to account for attainment of saturated wave amplitudes by the wave spectrum as a whole, yielding a saturation spectrum amplitude of $N^{2} / 6 \mathrm{~m}^{3}$ for velocities and a natural explanation for the evolution of the dominant vertical wave number, $m_{*}$, toward smaller $m$ with increasing altitude. Further refinements of this theory yielded a corresponding saturated amplitude for gravity wave temperature spectra of $N^{4} / 10 g^{2} m^{3}$ and implications for enhanced saturation and dissipation where $N$ increases with altitude [Fritts et al., 1988a; VanZandt and Fritts, 1989]. Attributes of this "linear saturation theory" include a simple conceptual mechanism for amplitude limits for superposed waves of various scales and frequencies, general agreement of the predicted spectral form and amplitude and variations of wave energy density with altitude with observations throughout the atmosphere [Allen and Vincent, 1995], and a natural explanation for the increase in the dominant vertical wavelength with increasing altitude [Tsuda et al., 1989]. Failings of the theory include an inability of the spectral description to account for specific wave sources having character quite different from the canonical spectral form, an inability to account for amplitudes in the stratosphere below the saturation limit because of wind shear effects on vertical wavelengths [Eckermann, 1995a; Alexander, 1996], relative amplitudes of velocity and temperature spectra that depart from predictions [Tsuda et al., 1991; Nastrom et al., 1997; de la Torre et al., 1999], and the neglect of wave-wave interactions that are known to cause spectral energy transfers. 
[153] An alternative explanation of the evolution of gravity wave spectral characteristics with increasing altitude was provided by Weinstock [1982, 1985, 1990] based on "nonlinear damping." Weinstock argued that this mechanism could both constrain wave amplitudes and account for spectral broadening with increasing altitude. Accounting for nonlinear influences within the wave spectrum, even without accounting explicitly for resonant interactions, was an appealing approach and spawned a number of extensions to the theory by $Z h u$ [1994], Gardner [1994], and Medvedev and Klaassen [1995]. As with linear saturation theory, however, the various extensions to Weinstock's theory have their detractors (see section 6.1), and clarification of the mechanisms controlling spectral amplitude and shape remains a high priority. Appealing aspects of the nonlinear damping theories include a recognition of the central role of nonlinearity in shaping the wave spectrum and approximate agreement with observed spectral shape and evolution with altitude. Liabilities include an inability to account explicitly for local wave field instability and turbulence and concerns by Hines [2002b] over the legitimacy of the underlying assumptions.

[154] A further approach to gravity wave saturation and spectral evolution was taken by Hines [1991, 1993, 1996], who modeled wave-wave interactions by "Doppler spreading" of any one member of the spectrum by all of the remaining members. The Hines theory relies on the Lagrangian description of wave-wave interactions by Allen and Joseph [1989] and shares the attributes and liabilities of this formalism discussed at length in section 6.1. This approach accounts qualitatively for increasing interactions and effects with increasing altitude and yields predictions of a tail spectrum that is also in approximate agreement with observations, subject to correction of the earlier estimates of spectral slope [Hines, 2001; Chunchuzov, 2002]. The theory has also led to the development of a gravity wave parameterization that has experienced a number of successes (see section 7) but has been criticized (see section 6.1) for simplifications in the underlying assumptions that are inconsistent with the claimed cascade toward smaller vertical scales. Perhaps its major contribution has been a focusing of attention and renewed research activity on the role of wavewave interactions in gravity wave spectral evolution in the atmosphere.

[155] A final approach to describing gravity wave spectral shape and evolution in the atmosphere is the "saturated-cascade similitude" theory by Dewan [1991, 1994, 1997]. The theory, as it has developed, embodies both the linear saturation ideas of Dewan and Good [1986] and Smith et al. [1987] as amplitude constraints and the notion that wave-wave interactions drive a cascade of wave energy from larger to smaller scales analogous to that observed in inertial-range turbulence. As in turbulence theory, spectral amplitudes are controlled by the energy dissipation rate $\epsilon$, which represents both energy input at large scales via vertical wave propagation and energy dissipation at sufficiently small scales. The theory is appealing in its simplicity and its dependence on scaling arguments, and it makes a number of predictions of 1-D spectra that are either in reasonable agreement with observations or pose observational tests of the theory. The suggestion that saturation and cascade dynamics are jointly at work in shaping the gravity wave spectrum is more realistic than theories relying largely on one or the other. Dewan's theory is also more in line with recent results by Sonmor and Klaassen [1997] indicating that local wave field instability and spectral energy transfers via wave-wave interactions are manifestations of related dynamics at different wave amplitudes. As such, further advances merging or generalizing saturation and cascade processes and effects may help to unify the divergent views of wave saturation and spectral evolution prevailing at present. There are, however, a number of shortcomings or uncertainties in the present formulation, hence motivation for further improvements to the theory. It is unclear but likely not the case that wave energy is transferred from large to small scales without dissipation, while the direction and/or efficiency of the cascade are uncertain, based on the discussion in section 6.1. Finally, the current theory does not predict 2-D spectra, although these would be valuable in assessing the interaction and cascade dynamics, and the prediction of the vertical wave number spectrum of vertical velocities has a slope of +1 which differs significantly from the various observations to date of order -1 to -3 [Kuo et al., 1985; Larsen et al., 1986; Wu and Widdel, 1990; Fritts and Hoppe, 1995].

\section{GRAVITY WAVE PARAMETERIZATIONS}

[156] The effects of gravity waves on the larger-scale circulation must be parameterized in global models because the spatial resolution required to model them directly is prohibitively fine. The waves important to the circulation in the atmosphere have horizontal wavelengths ranging from approximately tens to thousands of kilometers. The largest of these can be resolved in some global models; however, the vertical wavelength of a gravity wave will vary substantially with height owing to the effects of wind shear. Vertical wavelengths as small as 1-2 km are important to resolve in the lower stratosphere. Gravity waves with periods as short as $\sim 10 \mathrm{~min}$ can carry significant momentum flux vertically. Further, the sources of these waves include processes that are also parameterized and/or poorly resolved, namely, convective heating, fine-scale topography, localized shear zones, and frontal structures. The parameterizations for convection for example are designed for purposes other than generating a realistic wave spectrum [Ricciardulli and Garcia, 2000]. Direct modeling of a realistic gravity wave spectrum in global models is therefore still not feasible at the present time nor in the near future. Two effects of gravity wave dissipation that are currently 
believed to be necessary in global models are a body force on the background flow (important at the tropopause and throughout the middle atmosphere) and mixing effects on temperature and trace constituent distributions (important in the upper stratosphere and above).

[157] The essential ingredients of gravity wave parameterizations include specification of input parameters describing the gravity wave sources and estimation of the wave dissipation as a function of height. Observational constraints on parameterization inputs are, however, still lacking in necessary detail. There is also no current consensus on how to compute the dissipation with height. Therefore middle atmosphere models currently have an uncomfortably large degree of freedom in the parameterization of gravity wave effects.

[158] There are currently at least eight parameterization schemes proposed or in various stages of implementation in global models [Lindzen, 1981; Holton, 1982; Palmer et al., 1986; McFarlane, 1987; Fritts and Lu, 1993; Medvedev and Klaasen, 1995; Hines, 1997a, 1997b; Lott and Miller, 1997; Alexander and Dunkerton, 1999; Scinocca and McFarlane, 2000; Warner and McIntyre, 2001]. McLandress [1998] reviews the approach and basic mathematics for four of these. Here we will not embark on detailed descriptions of each parameterization. We will instead discuss the basic components of a gravity wave parameterization and describe some of the commonalities and differences among the various approaches in that context. The basic components are (1) specification of the characteristics of the waves at the source level, (2) wave propagation and/or spectral evolution as a function of height, and (3) wave dissipation and calculation of the effects on the background atmosphere.

\subsection{Parameterization of Gravity Wave Sources}

[159] Parameterizations of gravity wave effects all require input of the amplitudes and propagation properties of the waves at some altitude in the troposphere or at the model lower boundary. These would ideally be related to specific gravity wave sources as in the work of Rind et al. [1988], but this approach is insufficiently constrained by present observations. Stationary gravity waves $(c=0)$ generated by flow over topography are presently parameterized in many global models [e.g., Palmer et al., 1986; McFarlane, 1987; Miller et al. 1989]; however, the properties of other nonstationary gravity waves $(c \neq 0)$ and their sources are too poorly constrained to be included in this way. Instead, nonstationary waves, when they are included at all, are input with globally uniform properties or with properties that vary only as a function of latitude [Garcia and Solomon, 1985; Holton and Schoeberl, 1988; Smith and Brasseur, 1991; McLandress and Ward, 1994; Roble and Ridley, 1994; Norton and Thuburn, 1996; Mayr et al., 1997a; Manzini and McFarlane, 1998; Medvedev et al., 1998; Scaife et al., 2000].
[160] Linear gravity wave theory tells us that the required information about gravity wave sources includes their phase speeds, horizontal wavelengths, propagation directions, and momentum fluxes. (Note that horizontal wavelength or phase speed could be replaced with vertical wavelength or frequency using the dispersion relation (23).) A factor describing gravity wave spatial and/or temporal intermittency must also be considered because the waves with the specified properties will not generally be occurring throughout the model area at all times.

[161] Palmer et al. [1986] and McFarlane [1987] defined parameterizations for topographic wave sources. Momentum fluxes are related to the subgrid-scale orographic variance, atmospheric stability, and surface wind. The waves are launched propagating opposite to the surface wind with phase speed relative to the ground $c=0$. Bacmeister [1993] further considered the alignment of mountain ridges perpendicular to the surface wind. An efficiency factor is generally included because only a fraction of the surface stress will be carried by vertically propagating waves [Schär and Durran, 1997; Lott, 1998]. Horizontal wavelength is a free parameter, generally chosen to be $\sim 100 \mathrm{~km}$ or considered to be a tunable parameter.

[162] Kershaw [1995] describes a parameterization for momentum flux due to convectively generated gravity waves by assuming a mechanism analogous to topographic waves, but the waves are assigned a phase speed equal to the wind speed at the center of the convective layer. Chun and Baik [1998] describe a convective heat source parameterization that models convective heating as a steady process that similarly generates only waves that are stationary relative to the heat source. The horizontal wavelength spectrum that results is also highly dependent on a set of assumed properties of the convective heating that are currently poorly constrained. By contrast, other model studies [e.g., Piani et al., 2000] (also see Figure 5) instead suggest that in the absence of strong shear, convection may generate a very different spectrum of gravity waves with much larger phase speeds (see section 3.1).

[163] For nonstationary gravity waves $(c \neq 0)$, there is no consensus on the value of momentum flux at the source level, so it is yet another free parameter. Many model studies have not reported the value that has been assumed, making comparisons difficult. The best constraints on the momentum flux are indirect and are derived from estimates of the zonal mean wave-driven force that must be present to explain global temperature, wind, and trace constituent patterns. These constrain the gravity wave momentum flux crossing the tropopause and are described in section 8.1. A wide variety of gravity wave parameterizations are currently in use in global models. Intercomparison of these studies will begin to be possible when distributions of the crosstropopause gravity wave flux with latitude are reported, as Manzini and McFarlane [1998] have done. 
[164] Other properties of the spectrum of nonstationary waves input to parameterizations currently vary widely, and these differences may be of fundamental importance in explaining the differences among the results obtained. Some input an isotropic energy spectrum that is separable in $\hat{\omega}$ and $m$ with power law dependencies on these variables that resemble observations in the troposphere and stratosphere (see section 4) [Fritts and VanZandt, 1993; Fritts and Lu, 1993; Hines, 1997a, 1997b; Medvedev et al., 1998; Medvedev and Klaassen, 2000; Warner and McIntyre, 2001]. Hines [1997a] argues that the fundamental difficulty with this approach is that the low- $m$ portion of the spectrum is very poorly constrained by observations, yet it is this portion that has the largest effect in the mesosphere. The assumption of isotropy is also poorly constrained and probably wrong. Alexander and Dunkerton [1999] proposed a parameterization that can employ arbitrary source spectral shapes and anisotropies that are input as momentum flux versus phase speed. This approach suffers similar uncertainty in the input spectrum, particularly at high phase speeds (corresponding to low $m$ ), although model studies and observations of high phase speed waves generated by deep convection are beginning to constrain the phase speed spectrum of this source (see section 3.1.2).

[165] For nonstationary wave sources like convection the intermittency may be a small number, $\varepsilon \ll 1$, yet there are few observational constraints for this parameter. Alexander [1996] derived estimates of intermittency as a function of frequency and horizontal wavelength for high-frequency waves above deep convection in a cloudresolving model, and Zink and Vincent [2001b] estimated intermittency for low-frequency waves in midlatitude radiosonde observations. The values will depend on the bandwidths of the wave properties considered (e.g., $c, \hat{\omega}$, etc.) [Alexander and Dunkerton, 1999].

\subsection{Treatment of Wave Propagation and Dissipation}

\subsubsection{Commonalities and Differences}

[166] All parameterizations assume a set of gravity wave properties, specified either as a continuous wave spectrum or as a collection of discrete waves at some source altitude. The spectral properties of the sources may vary considerably among the different parameterizations, and except for topographic waves (input at the ground), the source altitude for nonstationary waves will additionally vary even among different applications of the same parameterization. Differences in the specification of gravity wave sources among parameterizations may be responsible for some large part of the differences in their effects seen in models. However, no controlled comparisons have yet been made that address this issue.

[167] All parameterizations are one-dimensional in that they assume the waves considered propagate only vertically and that only vertical variations in the background atmosphere influence wave propagation. All parameterizations use linear theory in the absence of dis- sipation and use the midrange frequency approximation to the linear dispersion relation (32) for at least some portion of the calculation. Total internal reflection is included via equation (30) in one [Alexander and Dunkerton, 1999], but this is generally only important for gravity waves with horizontal wavelengths shorter than $\sim 50 \mathrm{~km}$.

[168] Aside from gravity wave source specification, the main differences among parameterizations reside in their treatment of nonlinearity and mechanisms of wave dissipation. These differences result in different vertical variations in gravity wave effects.

\subsubsection{Treatment of Nonlinearity and Wave Dissipation}

[169] Most global model studies that have included gravity wave effects parameterized nonlinear effects using a scheme related to that proposed by Lindzen [1981] and formalized by Holton [1982]. These "Lindzen-type" parameterizations specify some discrete set of wave properties at a source level, use the midrange frequency approximation to calculate wave propagation with height, and then use the linear convective instability threshold to determine where wave dissipation will occur. Wave dissipation continues above the unstable level via a saturation condition (see Fritts [1984a] for a review). This basic formula is the basis of the widely used mountain wave parameterizations by Palmer et al. [1986] and McFarlane [1987], who added a Froude number condition for finding the altitude regions where wave saturation would occur. There are also nonstationary wave analogs to these mountain wave parameterizations [e.g., Kiehl et al., 1996; Norton and Thuburn, 1999].

[170] Alexander and Dunkerton [1999] used Lindzen's convective instability criterion but assumed total wave breakdown at that level rather than employing the wave saturation assumption. This simplification reduced computation time, allowing a treatment of a detailed spectrum of waves rather than the typical small set of 5-10 waves employed in Lindzen-type schemes. They also made explicit the separation of the parameters describing local wave momentum flux magnitude (which determines breaking levels) and net wave momentum flux (which is generally smaller if there is intermittency in the wave occurrence). The parameterization by Norton and Thuburn [1999] instead locked these two parameters together so that smaller fluxes gave higher breaking levels and vice versa. This is appropriate for mountain waves where amplitude and net flux are naturally linked because the intermittency in the source is modeled directly via topography and surface wind variations but is inappropriate for nonstationary wave sources with globally averaged input properties.

[171] Warner and McIntyre [2001] used the midrange frequency approximation to the linear dispersion relation and a saturation condition to limit wave amplitudes to a "quasi-saturation" spectrum. They developed a spectral analogue to the Lindzen [1981] saturation crite- 
rion and simplified the computation with a three-part spectrum approximation. Short horizontal wavelength waves in their source spectrum [Warner and McIntyre, 1996] carry significant momentum flux, so their neglect of wave reflection is a potentially serious problem. Fritts and VanZandt [1993] and Fritts and Lu [1993] used saturation theory and empirical constraints on the gravity wave spectrum to describe the spectrally integrated energy density and momentum flux as functions of height.

[172] Hines [1991, 1997a, 1997b] used linear theory and the midrange frequency approximation to describe wave propagation with height but treated dissipation as resulting from wave-wave interactions which cause Doppler-shifting and refraction of portions of the wave spectrum to vertical wavelengths shorter than a prescribed cutoff wave number where dissipation is assumed to occur. Medvedev and Klaassen [1995, 2000] instead treated dissipation via the nonlinear diffusion ideas of Weinstock [1982, 1990]. Both of these mechanisms have, however, been questioned in the recent literature (see section 6.1).

[173] Note that although many of the spectral gravity wave parameterization schemes [Fritts and $\mathrm{Lu}, 1993$; Medvedev and Klaasen, 1995, 2000; Hines, 1997a, 1997b; Warner and McIntyre, 1996, 2001] have been constrained to reproduce the "universal" $\mathrm{m}^{-3}$ gravity wave energy spectrum observed at high $m$ (section 4), these parameterizations can produce very different results (see section 8.4). This leads to the natural conclusion that the shape and magnitude of the spectrum at high $m$ alone do not sufficiently constrain parameterizations of gravity wave effects in the middle atmosphere.

[174] For a parameterization to be effective at both forcing the stratospheric QBO winds and driving the mesopause wind reversals, dissipation of nonstationary waves must occur for increasingly large intrinsic phase speeds and increasingly long vertical wavelengths at increasing altitudes. In other words, waves may dissipate fairly close to their critical levels in shear zones in the lower stratosphere but must dissipate far from their critical levels in the upper mesosphere. Intrinsic phase speed and vertical wavelength are related via equation (33). The result is that for nonstationary waves the force will tend to accelerate the mean wind (the force will have the same sign as the wind) in the lower stratosphere but decelerate the mean wind (the force will have opposite sign as the wind) in the upper mesosphere. (Note that this trend should not hold for the large amplitude mountain waves with phase speeds $c \sim 0$ that have been observed. Such waves can break and cause decelerations at all levels.) The potential for gravity waves to accelerate the mean wind is one reason to avoid the term "gravity wave drag" since this is an example of "gravity wave push.”

[175] The vertical variation in wave dissipation described above is a natural consequence of linear instability theory for small amplitude waves propagating through exponentially decaying atmospheric density and the observed zonal mean middle atmosphere winds [ $\mathrm{Al}$ exander and Rosenlof, 1996] (see also section 4). It can thus occur using any of the parameterizations above based on convective instability for some range of source spectrum properties. That includes the Lindzen-type, Warner and McIntyre [1996, 2001], and Alexander and Dunkerton [1999] parameterizations. It can also arise naturally in the Hines [1997a, 1997b] parameterization for wave dissipation because the amplitudes of the waves that drive the dissipation in this mechanism grow with altitude. They can thus shift waves with larger and larger intrinsic phase speeds (and vertical scales) to the prescribed dissipation scale as a function of height. The Hines dissipation scale is further allowed to vary with height adding another means of tuning this effect.

[176] Vertical gradients of gravity wave energy and momentum fluxes give rise to important terms in the energy and momentum conservation equations in global models (see section 8). All of the parameterizations will drive the deceleration of the winds in the mesosphere, but only a few have been shown to be effective at driving QBO circulations in global models. In addition to mean flow forcing effects of parameterized gravity waves, vertical mixing effects in the thermal energy and constituent transport equations are also important in the mesosphere of global models. Global model studies of both these effects are described in section 8 .

\section{INFLUENCES ON ATMOSPHERIC CIRCULATION AND STRUCTURE}

\subsection{Mean Flow Forcing Effects}

[177] Around 20 years ago, gravity wave effects on the zonal mean winds in the mesosphere and lower thermosphere were becoming widely appreciated. The gravity wave zonal mean forces cause reversals of the zonal mean jets and drive a mean meridional transport circulation that leads to a warm winter mesopause, a cold summer mesopause, and a reversal of the latitudinal temperature gradient expected in the absence of wave driving [Lindzen, 1981; Holton, 1982, 1983; Garcia and Solomon, 1985]. Gravity wave effects at slightly lower altitudes such as those driving the mesopause SAO [Dunkerton, 1982b] and QBO [Lindzen and Holton, 1968] had been proposed but were not generally accepted. Since that time, gravity wave effects at even lower altitudes, such as mountain wave drag effects on the circulation in the troposphere, have become widely accepted and are now included via parameterization in most global models to provide a drag force on the jet stream [Palmer et al., 1986; McFarlane, 1987; Miller et al., 1989]. Most recently, direct gravity wave effects in the stratosphere have been inferred. These effects include contributions to driving the tropical QBO [Gray and Pyle, 1989; Takahashi and Holton, 1991; Takahashi and Boville, 1992; Alexander and Holton, 1997; Dunkerton, 


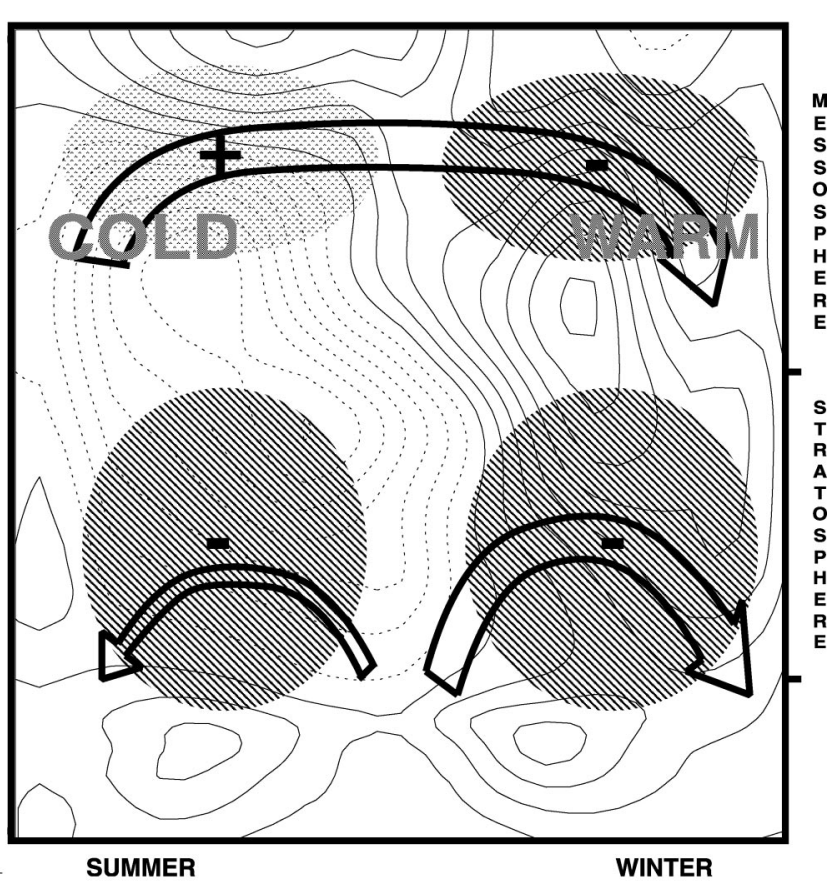

Figure 25. Schematic diagram illustrating gravity wave driving of the middle atmosphere transport circulation and effects on the zonal-mean extratropical winds and temperatures. The thin contours denote zonal mean wind at intervals of $10 \mathrm{~m} \mathrm{~s}^{-1}$ (dotted contours are for westward winds). The transport circulation is illustrated with thick arrows, and the gravity wave driving is illustrated with hatched areas with minus signs denote westward forcing and a plus sign denotes eastward forcing.

1997b; Scaife et al., 2000; Baldwin et al., 2001] and SAO (both in the stratosphere and mesosphere) [Hitchman and Leovy, 1988; Hitchman et al., 1992; Sassi and Garcia, 1994, 1997; Ray et al., 1998; Garcia and Sassi, 1999], contributions to driving the summer hemisphere equator to pole meridional transport circulation [Rosenlof, 1996; Alexander and Rosenlof, 1996], and effects on the winter polar vortex [Hitchman et al., 1989; O'Sullivan and Dunkerton, 1994; Kinnersley, 1996; Garcia and Boville, 1994]. Figure 25 illustrates these mean-flow forcing effects. The role of waves in the middle atmosphere transport circulation was reviewed by Holton and Alexander [2000]. The theory underlying wave driving of the transport circulation is embodied in the downward control principle [Haynes et al., 1991].

[178] In general, in the lower stratosphere the gravity wave mean body force will have the same sign as the local shear (and may or may not have the same sign as the local wind). Examples include the westward drag exerted by mountain waves at levels above the tropospheric jet peak winds and the QBO forcing that is eastward in eastward shear and westward in westward shear. In the mesosphere the force, in general, opposes the background winds. Wave amplitudes can grow large enough in the mesosphere that waves with large intrinsic phase speeds can break, while in the stratosphere, where wave amplitudes are smaller, breaking or dissipation will tend to occur only as waves approach their critical levels (see, e.g., equation (58)) unless the amplitudes are rather large.

[179] Several studies have estimated forces due to gravity waves in the stratosphere and mesosphere. Three basic techniques have been used: (1) Estimates of the zonal mean wave driving in the middle atmosphere, not explained by the observed planetary-scale wave driving, are presumed due to gravity waves. (2) Radar observations of zonal and meridional gravity wave momentum flux gradients employed in equation (42) give an estimate of gravity wave driving. (3) Atmospheric model studies with parameterized wave driving are constrained by observations to infer the contribution due to gravity waves.

\subsubsection{Zonal Mean Momentum Balance Studies}

[180] The first method estimates the gravity wave contribution to the wave driving through examination of the zonal mean momentum balance. The difference between the total momentum forcing and the resolved EP flux divergence contribution is presumed to be due to gravity wave driving. The total force is calculated using global satellite observations of temperature and the important radiatively active gases $\left(\mathrm{O}_{3}, \mathrm{H}_{2} \mathrm{O}\right.$, etc. $)$ input to a radiative transfer model to estimate the zonal mean radiative heating rate. This total force is what drives the zonal mean residual circulation (or transport circulation) in the middle atmosphere. Satellite temperature observations are then also used to compute global winds with a balance model, and the temperature and wind fields together are then used to define the planetary-scale wave fluxes and EP flux divergence. Examples of this method are described by Hamilton [1983], Smith and Lyjak [1985], Hitchman et al. [1989], Shine [1989], Marks [1989], Rosenlof [1995; 1996], and Fetzer and Gille [1996]. These studies have improved in accuracy over the years, partly because of improvements in the temperature and constituent measurements and partly through improvements in the models used to calculate the wind fields.

[181] Calculations of EP flux divergence give inherently noisy fields, so it is not surprising that most studies looking for the residual gravity wave term have focused on high altitudes near the stratopause and above where the gravity wave forcing becomes large [Hamilton, 1983; Smith and Lyjak, 1985; Shine, 1989; Marks, 1989; Fetzer and Gille, 1996]. At lower altitudes in the winter stratosphere the calculations of the residual gravity wave forcing have been highly variable, which could result both from errors in the calculations and true variability.

[182] Rosenlof [1996] and Alexander and Rosenlof [1996] focused on the summer stratosphere where resolved EP flux divergence is small and gravity wave driving is believed to dominate. A simple model of gravity wave propagation and dissipation was found to generate gravity wave driving in the summer strato- 
sphere that was consistent with the observational constraints. The model used a constant spectrum of nonstationary gravity waves input in the troposphere. Ray et al. [1998] applied the same methods in the equatorial stratophere and inferred that gravity wave driving is important to the eastward phase of the stratopause SAO. The gravity wave driving in the summer stratosphere is westward and below $\sim 1 \mathrm{hPa}$ has an estimated magnitude of order $\sim 1 \mathrm{~m} \mathrm{~s}^{-1} \mathrm{~d}^{-1}$. Similar westward forcing magnitudes have also been estimated near the tropopause in both summer and winter. The gravity wave driving at the onset of the stratopause SAO eastward phases (at equinoxes) has been estimated at $\sim 1-3 \mathrm{~m} \mathrm{~s}^{-1} \mathrm{~d}^{-1}$.

[183] Calculations of this type in the mesosphere extending up to $\sim 80 \mathrm{~km}$ altitude show the annual cycle in extratropical gravity wave driving of the summer-towinter pole-to-pole zonal mean circulation. The wave driving is westward in winter and eastward in summer [Shine, 1989; Marks 1989; Fetzer and Gille, 1996]. Two of these studies suggested that the mesopheric gravity wave driving in the Southern Hemisphere winter is significantly larger than that in the Northern Hemisphere winter. These estimates of the mesosphere force peak at magnitudes $\sim 30-60 \mathrm{~m} \mathrm{~s}^{-1} \mathrm{~d}^{-1}$ at solstice seasons. This hemispheric asymmetry seems opposite to that suggested by PMSE and temperature observations described in section 5 . The differences could suggest a high degree of variability or could be due to errors in the estimates.

\subsubsection{Radar Observations of Momentum Flux Convergence}

[184] Radar observations of gravity wave momentum fluxes at a number of sites have been used to estimate the body force due to gravity waves in the lower stratosphere and in the mesosphere and lower thermosphere. Mean momentum fluxes in the lower stratosphere over the MU radar (at $35^{\circ} \mathrm{N}$ latitude) were $\sim 0.1-0.3 \mathrm{~m}^{2} \mathrm{~s}^{-2}$, with peak values of $\sim 1 \mathrm{~m}^{2} \mathrm{~s}^{-2}$ and implied mean flow forcing of $\sim 1 \mathrm{~m} \mathrm{~s}^{-1} \mathrm{~d}^{-1}$ [Fritts et al., 1990b]. Momentum fluxes in the mesosphere and lower thermosphere have been measured more widely. At extratropical sites, mean values of $\sim 1-10 \mathrm{~m}^{2} \mathrm{~s}^{-2}$ have been measured with mean flow forcing in the range $\sim 10-70 \mathrm{~m} \mathrm{~s}^{-1} \mathrm{~d}^{-1}$ that generally oppose the mean winds [Reid and Vincent, 1987; Fritts and Yuan, 1989b; Tsuda et al., 1990b; Nakamura et al., 1993c]. At the tropical Jicamarca radar $\left(12^{\circ} \mathrm{S}\right)$ [Hitchman et al., 1992] fluxes of $1-4 \times 10^{-4} \mathrm{~Pa}\left(\sim 2-8 \mathrm{~m}^{2} \mathrm{~s}^{-2}\right)$ were observed with mean flow forcing $\sim 10-60 \mathrm{~m} \mathrm{~s}^{-1}$ $\mathrm{d}^{-1}$. Measurements at $35^{\circ}$ northern and southern latitudes have been compared [Nakamura et al., 1996], and like the residual force calculations, these suggest larger gravity wave driving in the Southern Hemisphere winter than in the Northern Hemisphere winter. Momentum fluxes observed in the mesosphere and lower thermosphere are highly variable, with peak values of $\sim 30-60$ $\mathrm{m}^{2} \mathrm{~s}^{-2}$ greatly exceeding the mean values. These imply correspondingly larger and possibly localized mean flow forcing and have been correlated with larger-scale motions [Fritts and Vincent, 1987; Fritts et al., 1992; Murphy and Vincent, 1998].

\subsubsection{Model Studies of Zonal Mean Wave Driving}

[185] A third method for estimating the gravity wave mean flow driving uses models of the middle atmosphere with parameterized radiative transfer and wave driving. The model results are then constrained by observations so that inferences about the gravity wave driving can be made. Holton [1982, 1983], Garcia and Solomon [1985], Holton and Schoeberl [1988], Hitchman et al. [1989], and Huang and Smith [1991, 1995] examined gravity wave driving at extratropical latitudes in the mesosphere. The gravity wave mean flow force in these models peaked at midlatitudes at solstice seasons with values ranging from $\sim 30$ to $120 \mathrm{~m} \mathrm{~s}^{-1} \mathrm{~d}^{-1}$, with more recent work tending to report smaller values.

[186] Other global and mechanistic model studies have focused specifically on the magnitude of the zonal mean transport circulation required to account for the summer mesopause thermal structure [Garcia, 1989; McIntyre, 1989; Fritts and Luo, 1995, Zhu et al., 1997]. These have suggested vertical velocities near the mesopause of $\sim 5 \mathrm{~cm} \mathrm{~s}^{-1}$ and meridional motions of $\sim 20-30$ $\mathrm{m} \mathrm{s}^{-1}$; values that are comparable to radar and satellite measurements of mean meridional winds near the polar summer mesopause. The observed meridional winds differ somewhat in their latitudinal distribution with peak magnitudes observed at higher and lower latitudes [Nastrom et al., 1982; Lieberman et al., 1998] than the model studies' inferred meridional transport circulation winds. Variability in wave driving can also account for abrupt temperature transitions that have been observed at the summer mesopause [Luo et al., 1995; Lübken, 1999].

[187] Dunkerton [1997b] modeled the QBO and SAO and inferred that gravity waves likely contribute at least half of the wave momentum flux needed to drive the QBO. His estimates of the cross-tropopause momentum flux carried by gravity waves with phase speeds in the range of QBO wind speeds were $\sim 2-3 \times 10^{-3} \mathrm{~Pa}$.

\subsection{Gravity Wave-Tidal-Planetary Wave Interactions}

[188] As discussed in section 2 and further in section 8.4, gravity waves play a central role in controlling the mean circulation and thermal structure through wave momentum transport, wave filtering, and the body forces arising from wave dissipation and momentum flux divergence. Such filtering and effects arise in response to mean shears as well as lower-frequency gravity waves (see section 6.1) and larger-scale tidal and planetary wave motions. The first studies along these lines were theoretical and addressed feedbacks on tidal structures due to selective gravity wave filtering near critical levels [Walterscheid, 1981], excitation of planetary waves at altitudes of asymmetric gravity wave body forcing imposed by variable source strengths [Holton, 1984], and 
damping of planetary waves in response to modulation of gravity wave filtering processes [Miyahara, 1985; Miyahara et al., 1986]. More recent model assessments of such interactions have revealed a number of potential influences on tidal and planetary wave structures and variability. Various studies suggest either tidal amplitude reductions [Forbes et al., 1991; Miyahara and Forbes, 1991; Lu and Fritts, 1993; Meyer, 1999a] or increases [Mayr et al., 1998], depending on which gravity wave parameterization scheme is used. However, Akmaev [2001] has argued that the Miyahara and Forbes [1991] results are due to an incorrect implementation of the Lindzen parameterization, while McLandress [2002] argues that gravity wave driving and induced diffusion are much less important in controlling tidal amplitudes than other nonlinear interaction and advection processes.

[189] Several studies considered the implications of tidal modulation of gravity wave-induced diffusion and/or momentum deposition, arguing that these may be important in minor species chemistry and the creation of temperature inversions [Forbes et al., 1991; Liu et al., 2000], in altering mean momentum deposition [ $L u$ and Fritts, 1993; McLandress and Ward, 1994], and in enhancing planetary wave excitation in the mesosphere in response to spatially localized orographic sources [McLandress and Ward, 1994]. Other studies suggested that seasonal variations in mean winds enable a gravity wave modulation of tidal amplitudes that accounts for the observed semiannual variations [Mayr et al., 1998; Meyer, 1999a] and that filtering of gravity waves by planetary waves at lower altitudes imposes a periodic forcing near the mesopause which enables 2-day and 16-day wave influences to penetrate well into the thermosphere [Meyer, 1999b; Norton and Thuburn, 1997].

[190] All of the above studies exhibited complex coupling between the imposed spectrum of gravity waves and the larger-scale motions accounting for wave filtering. In particular, tidal amplitude changes accompanying gravity wave-tidal interactions were found to be sensitive not only to geophysical parameters but also to the specific gravity wave parameterization employed. This is because tidal amplitudes (as well as any other variable wind field accounting for gravity wave filtering, i.e., mean winds, planetary waves, or the semiannual and quasi-biennial oscillations) respond to gravity wave filtering and momentum flux divergence differently depending on the phase of the gravity wave forcing relative to the phase of the filtering wind field, which can differ between parameterizations. If parameterized gravity waves are dissipated near and below the wind maxima (in the direction of gravity wave propagation), then the result is a wind acceleration and an induced downward phase motion (as discussed in section 7.2.2). If, on the other hand, parameterized gravity waves dissipate by breaking (due to amplitude growth with height where the winds are a minimum or where intrinsic phase speeds are large), then gravity wave forcing reduces wind amplitudes and the induced phase motion depends on where forcing occurs relative to the wind maxima. For these reasons, comparisons with observations may pose important constraints on the gravity wave parameterizations underlying such interactions in numerical models.

[191] Observational studies were slower to yield insights into gravity wave interactions with tidal and planetary wave motions. Nevertheless, several studies have shed light on the degree of modulation of gravity wave amplitudes and fluxes and their potential influences on the structures responsible for wave filtering. Evidence of gravity wave-tidal and gravity wave-planetary wave interactions has come primarily from various radars spanning equatorial to high latitudes. Measurements of gravity wave momentum fluxes and tidal amplitudes at Adelaide, Australia, and Poker Flat, Alaska, revealed a tendency for an out-of-phase relationship between fluxes and tidal winds [Fritts and Vincent, 1987; Wang and Fritts, 1991], a potential for very large momentum flux modulations correlated with large tidal amplitudes and shears (as large as $\sim 40 \mathrm{~m}^{2} \mathrm{~s}^{-2}$ ), and a reduction of tidal amplitudes accompanying strong gravity wave filtering [Fritts and Vincent, 1987]. An example of the modulation of gravity wave momentum fluxes by the diurnal tide for 3 days during which the tide was strong is shown in Figure 26. In this example, the flux estimates are 8-hour averages, the modulation increases with altitude, and the peak values are $\sim 10$ times as large as the mean momentum flux [Fritts and Vincent, 1987]. A similar modulation of momentum fluxes by the 2-day wave was suggested by the observations of Murphy and Vincent [1998], while Thayaparan et al. [1995], Isler and Fritts [1996], and Manson et al. [1998b] noted significant peaks in gravity wave variances at the dominant tidal and planetary wave periods, suggestive of strong mutual interactions. Finally, indirect evidence of gravity wave filtering by planetary waves and of gravity wave influences on tidal structures has also been obtained. Smith [1996] inferred gravity wave filtering by and forcing of planetary waves from out-of-phase zonal wind anomolies at stratospheric and mesospheric altitudes, respectively, while Nakamura et al. [1997] inferred gravity wave-tidal interactions from tidal structure and variability at multiple equatorial and subtropical radar sites. There is insufficient data at this stage, however, to be able to distinguish between or guide the development of more quantitative gravity wave parameterizations.

\subsection{Turbulent Mixing and Transport}

[192] The impact of turbulence arising from gravity wave instability processes is one of the least quantified aspects of gravity wave forcing of the middle atmosphere at present. In fact, there are two separate issues. One is the energy dissipation rate $\epsilon$ accompanying gravity wave instability and its spatial and temporal variability; a second is the efficiency of mixing that occurs for a fixed energy dissipation rate. The former is becoming better known as measurements and theoretical inferences are beginning to converge. In the stratosphere, both $\epsilon$ and 


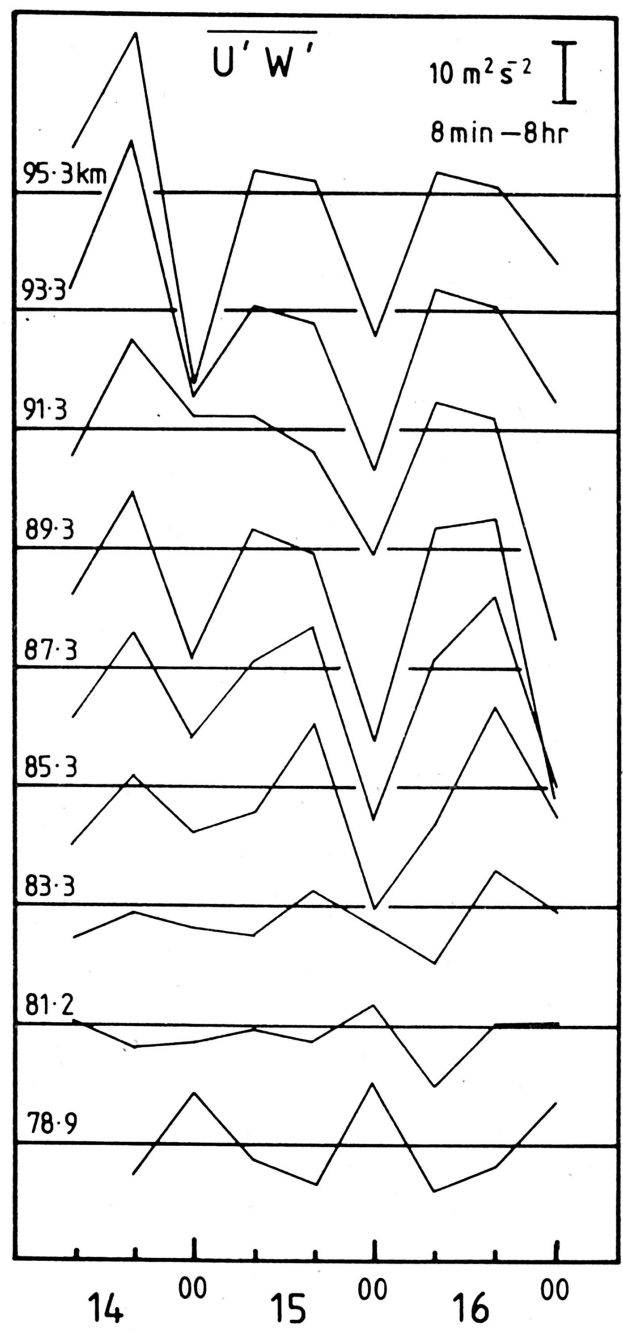

Figure 26. Zonal gravity wave momentum fluxes for 8-hour data segments during 3 days of large diurnal tidal winds over Adelaide, Australia. Note that peak momentum fluxes are $\sim 10$ times mean values. After Fritts and Vincent [1987] (reprinted with permission of the American Meteorological Society).

turbulent mixing and transport are relatively small and have minor impact on the large-scale thermal and constituent structures. Because, however, gravity wave energy flux and energy dissipation rate increase rapidly with altitude (see equations (51) and (52)), their influences are greater in the mesosphere and lower thermosphere. Early estimates of $\epsilon$ at higher altitudes based on radar spectral width and rocket trail diffusion measurements tended to be very high, but more recent estimates are typically $\sim 10-300 \mathrm{~mW} \mathrm{~kg}^{-1}$ at altitudes from $\sim 80$ to $110 \mathrm{~km}$ [CIRA, 1986; Hocking, 1990]. More recent in situ measurements have refined these estimates even further, lowering mean $\epsilon$ estimates at high latitudes in winter below previous values and suggesting a sharp peak in $\epsilon$ at the high-latitude summer mesopause [Blix et al., 1990; Lübken et al., 1993; Lübken, 1997]. Peak values in these estimates are $\sim 200 \mathrm{~mW} \mathrm{~kg}{ }^{-1}$ and are in reasonable agreement with earlier CIRA 86 estimates. Peak values are also in reasonable agreement with recent theoretical estimates based on mean gravity wave energy densities [Fritts and VanZandt, 1993; Hall and Hoppe, 1998] and with estimates based on the gravity wave forcing required to account for the summer mesopause thermal structure [Fritts and Luo, 1995]. Comparable estimates have arisen from in situ measurements at equatorial latitudes [Goldberg et al., 1997], while Fuller-Rowell [1994] attributed observed latitudinal variations in nitric oxide to a latitudinally varying turbulent transport with a maximum at midlatitudes.

[193] As noted in section 8.3, several studies have anticipated more efficient turbulent transport of momentum than of heat and constituents based on localization of turbulence within the wave field [Fritts and Dunkerton, 1985; Coy and Fritts, 1988; McIntyre, 1989; Gavrilov and Yudin, 1992] and assessments of the turbulent transport required to account for observed thermal and constituent profiles [Strobel et al., 1985, 1987; Holton and Schoeberl, 1988; Smith and Brasseur, 1991]. More efficient turbulent transport of momentum than of heat implies a turbulent Prandtl number significantly larger than unity, with expected values in the range $P r \sim 3-10$. The implication of a large $P r$ is a significantly reduced turbulent diffusivity, $K_{z z}$, relative to that anticipated from energy dissipation rates inferred from either discrete wave or spectral descriptions of gravity wave dissipation [Lindzen, 1981; Fritts and VanZandt, 1993]. These gravity wave dissipation studies together with more recent numerical modeling of wave breaking and turbulence dynamics [Fritts et al., 2003] suggest that the turbulent Prandtl number will depend on wave amplitude and intrinsic frequency, with more efficient mixing accompanying larger wave amplitudes and more vigorous turbulence. We also anticipate that turbulent mixing accompanying $\mathrm{KH}$ instability of the wave field may imply smaller $P r$ since in this case strong mixing occurs in a sheared and stratified environment. Our understanding of the details of turbulence generation and mixing is insufficient, however, to be able to quantify middle atmosphere effects more completely at this time.

\subsection{General Circulation Model Studies}

[194] Several studies have used high-resolution GCMs to examine the properties of gravity waves resolved in these models and to examine their effects on the middle atmosphere circulation. Experiments with the SKYHI GCM [Hamilton, 1995, 1996; Jones et al., 1997] have shown a tendency toward better simulation of the middle atmosphere circulation with increasing latitude and longitude resolution from $3^{\circ} \times 3.6^{\circ}$ to $0.6^{\circ} \times 0.72^{\circ}$. The spectrum of wave momentum flux versus zonal wave number in these simulations is rather flat, suggesting that even wave numbers beyond 140 would need to be resolved to capture the wave fluxes important to the middle atmosphere circulation. The conclusion of these studies is that gravity wave effects in middle atmosphere GCMs must be included via parameterization at least for the foreseeable future. 
[195] A number of studies have applied parameterizations of gravity wave effects in GCMs in order to study the role of gravity waves in various phenomena important to middle atmosphere dynamics. The results of such experiments are often difficult to interpret because they are complex in that they include many parameterized processes and modes of interaction. Insights gained from such experiments are described in this section.

\subsubsection{Mesospheric Circulation}

[196] Every gravity wave parameterization in use has been designed to yield the summer eastward and winter westward momentum forcing in the mesosphere that drives the pole-to-pole residual circulation (section 8.1). This role for gravity waves in the mesosphere was recognized decades ago [Lindzen, 1973, 1981; Holton, 1982] and has more recently been validated by full GCM studies [Roble and Ridley, 1994; Norton and Thuburn, 1996; Hamilton, 1997; Manzini and McFarlane, 1998].

[197] Two recent studies considered the effects of asymmetries in the eastward and westward gravity wave momentum fluxes input via parameterization but came to opposite conclusions. Manzini and McFarlane [1998] launched an isotropic spectrum of waves at two different source levels (at the ground and near the tropopause) in the MA/ECHAM4 model using the Hines [1997a, 1997b] parameterization. Launching the waves at the ground led to smaller eastward and larger westward momentum flux crossing the tropopause at midlatitudes because of filtering of eastward waves by the tropospheric jets (Figure 27). The larger westward fluxes in turn produced more realistic middle atmosphere zonal mean winds. Medvedev et al. [1998] applied their gravity wave parameterization [Medvedev and Klaassen, 1995] in the CMAM model. They compared results with an isotropic tropopause launch spectrum, with phase speeds in the range 0 $<c<60 \mathrm{~m} \mathrm{~s}^{-1}$ in four azimuths, to results with only the eastward propagating waves with larger fluxes. The results with eastward waves alone showed weaker and more realistic zonal mean winds in both summer and winter (Figure 28). This is an apparently paradoxical result since the westward forcing needed to decrease the winter jet strength would be expected to decrease in the anisotropic case given the physics embodied in equation (42).

[198] Norton and Thuburn [1996, 1997] applied a Lindzen-type parameterization of gravity wave forcing in the UGAMP GCM. They found the model with gravity wave forcing developed a realistic 2-day wave while no 2-day wave developed in the model with only Rayleigh friction. They concluded that gravity wave forcing in their model was essential to maintaining the unstable zonal mean state that led to the 2-day wave generation.

[199] Several studies have employed mechanistic versions of GCMs to study the effects of various gravity wave parameterization schemes in the mesosphere. A comparison of the effects of the Hines [1997a, 1997b] and Fritts and $L u$ [1993] parameterizations on the diur-
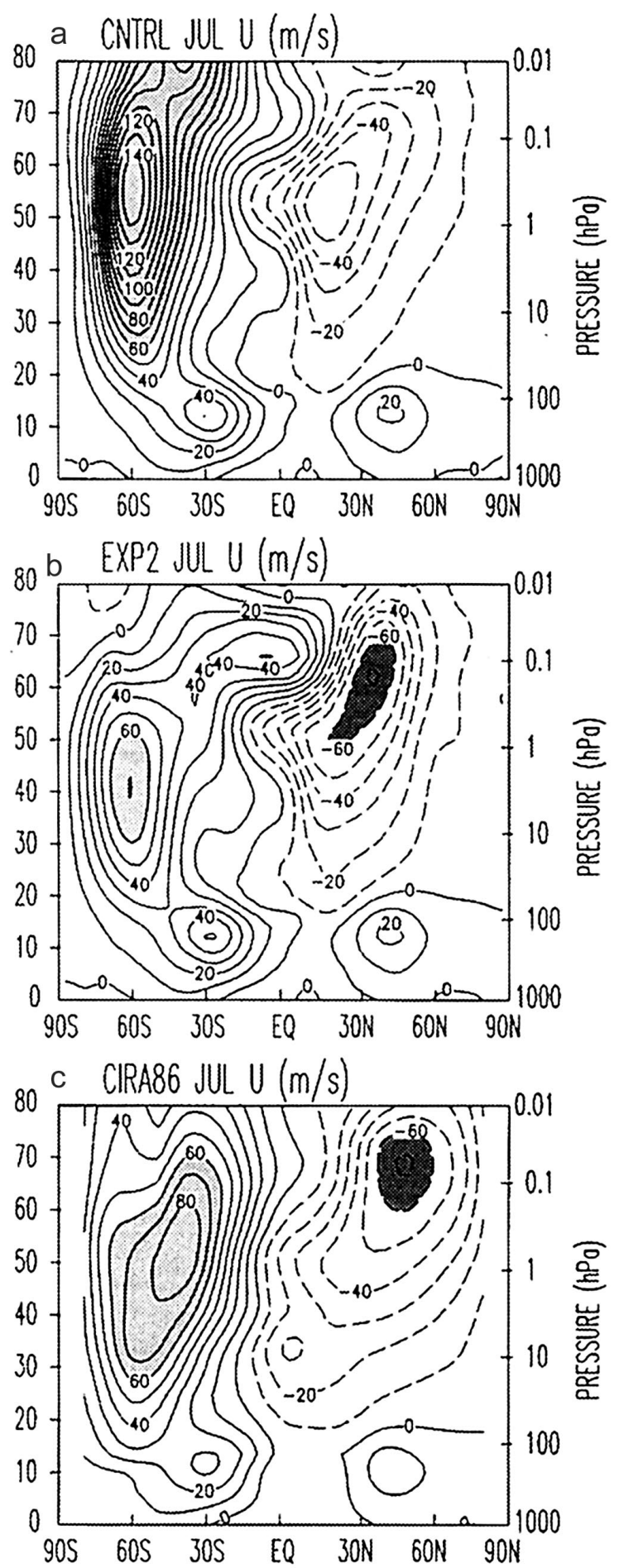

Figure 27. July zonal mean winds resulting from the ECHAM4 GCM with (a) approximately isotropic gravity wave fluxes input at the tropopause and (b) anisotropic gravity wave fluxes at the tropopause with smaller eastward gravity wave fluxes at midlatitudes. (c) The climatology from CIRA [Fleming et al., 1988]. After Manzini and McFarlane [1998].

nal tidal structure suggested that the Hines scheme tended to amplify tidal amplitudes, while the Fritts and $\mathrm{Lu}$ scheme caused excessive dissipation of the tides [McLandress, 1998]. Other mechanistic and simplified 


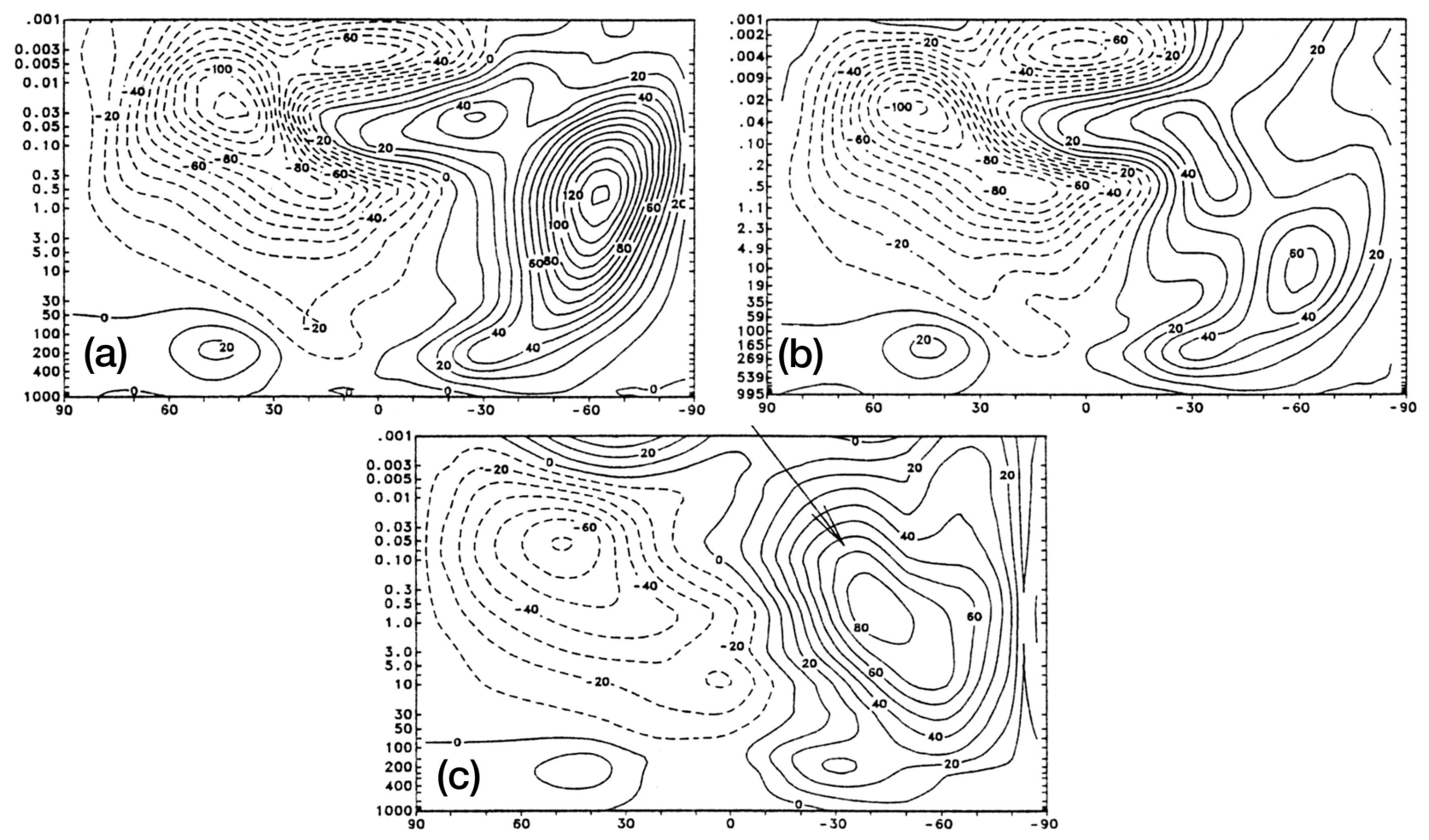

Figure 28. July zonal mean winds resulting from the CMAM GCM with (a) isotropic gravity wave sources input near the tropopause and (b) anisotropic gravity wave sources at the tropopause with larger eastward fluxes at all latitudes. (c) The climatology from CIRA [Fleming et al., 1988]. After Medvedev et al. [1998].

GCMs have been used to test gravity wave parameterization effects in the mesosphere [Hamilton, 1997].

\subsubsection{High-Latitude Stratosphere}

[200] Through the concept of "downward control" of the mean meridional circulation by wave-induced mean zonal forces [Haynes et al., 1991], Garcia and Boville [1994] stress that the winter mesospheric gravity wave forcing can have effects down to $\sim 30 \mathrm{~km}$ in the highlatitude stratosphere that can help to alleviate the "cold pole" problem that plagues GCM studies of the winter stratosphere. Hamilton [1997], however, also suggests that gravity wave forcing at stratospheric altitudes, although smaller in magnitude, is also important to the winter circulation.

[201] The role of gravity wave forcing in sudden stratosphere warming (SSW) events has been examined in several global model studies. Lawrence [1997] compared effects of Hines and Fritts and Lu parameterization on the winter stratosphere. Both parameterizations generated reasonable zonal mean mesosphere winds, but the Fritts and $\mathrm{Lu}$ parameterization was found to inhibit the occurrence of SSW events while Hines did not. Pawson [1997] found that topographic gravity wave drag in the lower stratosphere influenced the onset of SSW events but found little or no sensitivity to mesospheric drag parameterized as Rayleigh friction. Rind et al. [1988] found that gravity wave forcing experiments, designed to describe waves generated by different sources, affected both the character and existence of SSW events in the GISS GCM.

\subsubsection{Equatorial Oscillations}

[202] At the equator, where the Coriolis force vanishes, the atmospheric response to a zonal force is simply zonal acceleration. Dissipation of zonally propagating waves at tropical latitudes provides the forcing that drives or helps to drive the three dominant equatorial oscillations: (1) the QBO in the lower stratosphere, (2) the stratopause SAO, and (3) the mesopause SAO. Both gravity waves and planetary-scale waves likely participate in driving all three of these oscillations to differing degrees. What remains to be quantified is the spectrum of wave momentum flux in the tropics. Global model studies are therefore free to include adjustable parameterized gravity waves without adequate constraints either on the gravity wave fluxes or on the resolved planetary-scale wave fluxes. Mayr et al. [1997b] demonstrated the potential of the gravity-wave-driving mechanism for all three of these equatorial oscillations in a simplified global model with parameterized gravity wave fluxes.

[203] The QBO in the lower stratosphere zonal winds is a wave-driven phenomenon believed to be predominantly driven by dissipation of planetary-scale waves [Baldwin et al., 2001]. However, zonal forcing accompanying the dissipation of gravity waves is likely to contribute significantly, with recent estimates suggesting they may transport roughly half of the flux [Dunkerton, 1997; 
Piani et al., 2000]. Until very recently, obtaining a QBO in GCM studies had proved difficult. Recent GCM studies with increased horizontal and vertical resolution and decreased numerical diffusion have since developed QBO-like oscillations [Takahashi, 1996; 1999; Horinouchi and Yoden, 1998; Hamilton et al., 1999]. These successes are believed to be due to the increase in tropical wave fluxes allowed by the finer resolution and reduced numerical dissipation [Boville and Randel, 1992; Nissen et al., 2000]. Scaife et al. [2000] instead modeled a QBO in a GCM by including parameterized small-scale gravity wave fluxes.

[204] The SAO at the stratopause is driven in part by the meridional advection of summer hemisphere westward (easterly) winds across the equator and in part due to zonal wave driving particularly important during the eastward (westerly) wind phases of the SAO [Sassi et al., 1993; Hamilton et al., 1995; Müller et al., 1997; Garcia et al., 1997]. Rind et al. [1988], Jackson and Gray [1994], and Medvedev and Klaasen [2001] modeled realistic SAOs by including parameterized gravity wave fluxes, the latter with a model deep enough to include an SAO at the mesopause.

\section{SUMMARY, NEEDS, AND RECOMMENDATIONS}

[205] Our intent in this review was to provide a broad overview of the current understanding of gravity wave dynamics and effects in the middle atmosphere. Considerable research activity in this area over the past two decades made this a daunting undertaking. In choosing to be comprehensive in scope we have necessarily provided less discussion and fewer references in many areas than we would have preferred, emphasizing the more significant contributions and directing readers, where appropriate, to related reviews having more limited foci.

[206] We began our review with a derivation of the dispersion and polarization relations using linear theory and with a discussion of the simplifications and dynamics appropriate for various ranges of intrinsic frequency. These offer, in most cases, significant insights into wave structure and effects at a small cost in accuracy. Also noted were departures from the expectations of linear theory accompanying wave transience, spatial localization, or finite amplitude effects that may have important implications for the middle atmosphere. Particularly relevant here are self-acceleration effects that enable wave packets to penetrate beyond the turning and critical levels anticipated by linear theory, with corresponding implications for wave dissipation and momentum transport. The impact of such effects in the middle atmosphere is not yet known, but studies addressing these effects are underway.

[207] We then summarized what is known of the more important sources of gravity waves, dominant wave scales, and factors influencing wave propagation in variable environments. Theoretical, modeling, and observa- tional studies have yielded considerable advances in quantifying the mechanisms of and responses to wave forcing due to topography, convection, wind shear, geostrophic adjustment, body forcing, and wave-wave interaction sources. Mountain waves occur on scales dictated by the topography, may propagate to high altitudes, and have peak momentum fluxes at horizontal scales of $\sim 10-100 \mathrm{~km}$. Convection excites gravity waves having a wide range of spatial scales and phase speeds via several mechanisms that are dependent on the cloud environment and which exhibit significant temporal variability. Wind shear appears to support envelope radiation of gravity waves on the scale of patches of KelvinHelmholtz instability with attributes that favor their penetration to greater altitudes, while geostrophic adjustment favors inertia-gravity wave radiation due to the slow timescale and large spatial scale of the adjustment process. Body forcing due to local gravity wave dissipation and momentum flux divergence, in contrast, may occur on fast or slow timescales and on many spatial scales, resulting in a spectrum of radiated waves that mirror the source characteristics. Finally, wave-wave interactions are operative throughout the atmosphere, with interaction rates increasing with wave amplitude and energy transfers toward both larger and smaller scales. Dominant scales, amplitudes, and phase speeds are dictated largely by the various gravity wave sources at lower altitudes but are increasingly determined by propagation effects, filtering, and wave-wave interactions at greater altitudes. As altitude increases, so do the dominant vertical scales because wave amplitudes at smaller vertical scales are constrained by saturation processes whereas amplitudes at larger vertical scals are not. Despite significant advances, there remain many aspects of gravity wave sources and propagation that require further quantification. These are delineated below.

[208] Broad consistency in spectral shape and evolution with altitude led naturally to a spectral description of gravity wave motions. While far from the universal spectrum advocated initially, the frequency and vertical wave number spectra of horizontal velocity and temperature have near-universal forms throughout the middle atmosphere. Such descriptions constrain wave amplitudes, fluxes, and parameterizations and offer insights arising from spectral character and variability (or lack thereof). We also noted, however, that spectra often conceal, rather than reveal, the dynamics underlying spectral shape and pointed out where caution is warranted. Present unknowns include the degree to which adherence to or departures from specific spectral shapes are indicative of specific dynamics (e.g., saturation processes) or whether other spectral descriptions (e.g., wavelet spectra) might be more enlightening, given the localized nature of gravity waves in space and time that is often observed.

[209] Climatologies of gravity wave velocity and temperature perturbations have been compiled; however, these do not yet adequately constrain the inputs to 
gravity wave parameterizations for global models. What is needed are descriptions of gravity wave sources and the detailed properties of the waves generated. The gravity wave climatologies instead have primarily served to partially validate the use of linear models for describing gravity wave propagation through the middle atmosphere and interaction with the background atmosphere with height. Departures from linear theory have been treated in these models with dissipation that is tied to linear instability or saturation of wave amplitudes. These treatments, of course, have serious limitations that are underscored by the more detailed studies of wave instability and mean flow interactions summarized below. The linear theory, however, remains a valuable research tool and is the starting point for most gravity wave parameterization approaches.

[210] Patterns observed in gravity wave climatologies are also influenced by the range of gravity wave properties each observation method is capable of detecting. The climatology of gravity waves that is ultimately needed will have to be constructed from a variety of measurement techniques, with modeling tools employed to aid in the interpretation of the observations.

[211] We next surveyed recent work addressing wavewave interaction, wave instability, and wave saturation processes. These studies have yielded dramatic advances in our understanding of the myriad types of instabilities to which gravity waves are susceptible. Notable findings include the identification of wave-wave interactions that are facilitated by mean shear, the occurrence of wavewave interactions (at large wave amplitudes) on a timescale competitive with local wave field instability, and characterization of the spectral energy transfers due to wave-wave interactions. Other studies identified links between instabilities occurring at small and large wave amplitudes, instability processes due to finite amplitude wave packets via wave-mean flow interactions, and definition of the instability dynamics leading to and occurring within a turbulence cascade. Despite these advances the competition between wave-wave interactions and local instability dynamics and their respective control over spectral shape and amplitude remain major unknowns.

[212] The last two decades also saw the development of several classes of saturation theories and a number of attempts to distinguish the most relevant dynamics. The major contenders are linear saturation theory (with amplitude constraints due to local instability), various nonlinear diffusion theories (relying on wave-wave interactions for spectral transfers and wave amplitude limits), Doppler spread theory (describing nonlinear interactions as advection by other spectral members, assuming they all occupy a common volume in physical space), and saturated cascade theory (employing elements of local instability and wave-wave interactions). Deciding which theories are most realistic has proven difficult, however, because all yield estimates of spectral amplitude and shape that are arguably consistent with the various mea- surements available. Perhaps more importantly, all of these theories are at present incomplete because they all fail to incorporate quantitatively the two nonlinear effects known to occur, i.e., wave-wave interactions and local wave field instability. As such, it is likely more valuable to recognize the insights we have gained from each rather than attempt to identify the "most correct" among deficient or incomplete alternatives. The result is a recognition of the dual role of nonlinearity, via both wave-wave interactions and local instability, in causing wave saturation and in determining the spectral shapes that arise from these dynamics. Thus a complete saturation theory will almost assuredly not be analytic but will rely instead on numerical studies of the competition among or merging effects of dynamics that are now believed to be linked theoretically.

[213] Parameterizations of middle atmosphere gravity wave effects presently suffer acutely from the lack of constraints on their inputs and tunable parameters. Global modelers can use these poorly constrained parameterizations, given the wide range of free parameters available, to get almost any answer they require. Until the needed constraints on parameterization inputs are forthcoming, the differences between different methods of parameterizing gravity wave dissipation and interaction effects will be difficult or impossible to quantify. The process of tuning gravity wave parameterizations in global models to attempt to infer the properties of the gravity wave input parameters is unwise at present because the range of parameters is too broad, and the solutions are not unique. As a result, GCMs tuned for one result (and at one resolution) using a gravity wave parameterization cannot confidently be used in any prognostic way.

[214] There has been significant progress in quantifying gravity wave-mean flow effects over the last two decades. The important mechanisms of wave dissipation are, however, still being debated and likely vary with altitude through the middle atmosphere. In the case of wave breaking, the mixing effects appear to be highly sensitive to the detailed properties of the wave at the point of breakdown. Tidal and planetary wave interactions likewise prove to be highly sensitive to details of the gravity wave field that are not known. To parameterize these effects therefore requires much more extensive knowledge of the properties of gravity waves in the middle atmosphere and of their sources than was imagined 20 years ago.

[215] Gravity waves almost certainly play a role in driving the prominent $\mathrm{QBO}$ and $\mathrm{SAO}$ equatorial wind oscillations. Quantifying that role remains illusive, however, until more complete knowledge is attained of the momentum flux spectrum of tropical wave motions ranging from planetary-scale to small-scale gravity waves. Similar lack of quantification of gravity wave fluxes and other gravity wave properties at high latitudes also hinders our ability to quantify their role in extratropical 
processes such as sudden stratospheric warmings and the wave-driven transport circulation.

[216] Gravity wave interactions with larger-scale waves are strong and have been studied numerically and observationally. However, the details of the interactions appear to be highly sensitive to the relative phase of the gravity wave dissipation and the larger-scale wind variations with height. These remain poorly understood, as is evident from the fact that different gravity wave parameterizations yield very different results.

[217] Our field has experienced dramatic advances in understanding the dynamics and effects of middle atmosphere gravity waves over the last two decades. It is also clear that we require further advances in a number of areas before we can claim a comprehensive understanding of gravity wave dynamics and an ability to account for these dynamics statistically in large-scale operational or research models. We offer here our view of the further advances that are most needed to achieve these goals.

1. Observations and modeling studies that characterize and quantify the dominant gravity wave sources, especially scales, phase speeds, and momentum fluxes, as functions of meteorological conditions are a high priority, as this information underlies any attempt to quantify gravity wave influences at greater altitudes. More detailed observations and numerical studies are also required to quantify wave intermittency imposed by variable sources, propagation conditions, etc., as intermittency has major implications for wave effects at greater altitudes, including instability processes, wavewave and wave-mean flow interactions, and radiation of secondary waves.

2. Further studies are also required to define the interplay of and competition among the nonlinear processes controlling spectral evolution with altitude in various environments, as these control gravity wave propagation, dissipation, and forcing at greater altitudes. These advances are likely to require numerical studies, as current and anticipated observational capabilities are unlikely to permit complete specification of the motion fields and their evolution in three dimensions. For the same reasons, numerical studies are required to characterize fully the mixing dynamics and transport accompanying the various wave instability processes leading to turbulence, including the statistical effects of multiple waves and events.

3. Finally, the accurate parameterization of gravity wave effects remains a critical need, as these may not ever be adequately described directly in large-scale models. New parameterizations will need to describe gravity wave sources, propagation and filtering, wave-wave (including tidal and planetary wave) interactions, spectral evolution, mean and variable climatologies, and instability and mixing far more quantitatively than present schemes. It seems unlikely, in fact, that any single scheme will satisfy all of these needs and that different schemes will be required to characterize different dy- namics. Importantly, advanced parameterizations will rely to a large degree on the needed observational, numerical, and theoretical advances noted above and will advance together with successes in these areas.

[218] Recent theoretical and numerical activities have clarified key concepts, defined important dynamics and effects, and highlighted unresolved issues. Atmospheric and laboratory measurements have yielded similar advances in quantitative measurement techniques and definition of gravity wave sources, propagation characteristics, climatologies, instability processes and dissipation, mean and large-scale wave forcing, and variability. Ongoing and future research promises additional advances and further quantification of gravity wave dynamics and effects. Of the major needs identified above, there are none that seem insurmountable, given a continuation of our successes to date and the increasing awareness of the importance of the field, and we look forward to vigorous and stimulating interactions in achieving our collective objectives in the future.

[219] ACKNOWLEDGMENTS. D.C.F.'s review effort was supported by the National Science Foundation under grants ATM-9618004, ATM-9708633, ATM-9816160, the Air Force Office of Scientific Research under contracts F49620-98-C0029 and F49620-00-C-0008, and the National Aeronautics and Space Administration under contracts S-46579-G and NAG502036. Support for M.J.A. came from the National Science Foundation grant ATM-9907501 and NASA contract NASW01017. The authors would like to thank Jim Holton, Bob Vincent, and two anonymous reviewers for valuable comments on the first draft.

\section{REFERENCES}

Adrian, R. J., C. D. Meinhart, and C. D. Tomkins, Vortex organization in the outer region of the turbulent boundary layer, J. Fluid Mech., 422, 1-54, 2000.

Akmaev, R. A., Simulation of large-scale dynamics in the mesosphere and lower thermosphere with the Dopplerspread parameterization of gravity waves, 2, Eddy mixing and the diurnal tide, J. Geophys. Res., 106, 1205-1213, 2001.

Alexander, M. J., A simulated spectrum of convectively generated gravity waves: Propagation from the tropopause to the mesopause and effects on the middle Atmosphere, $J$. Geophys. Res., 101, 1571-1588, 1996.

Alexander, M. J., Interpretations of observed climatological patterns in stratospheric gavity wave variance, J. Geophys. Res., 103, 8627-8640, 1998.

Alexander, M. J., and T. J. Dunkerton, A spectral parameterization of mean-flow forcing due to breaking gravity waves, J. Atmos. Sci., 56, 4167-4182, 1999.

Alexander, M. J., and J. R. Holton, A model study of zonal forcing in the equatorial stratosphere by convectively induced gravity waves, J. Atmos. Sci., 54, 408-419, 1997.

Alexander, M. J., and L. Pfister, Gravity wave momentum flux in the lower stratosphere over convection, Geophys. Res. Lett., 22, 2029-2032, 1995.

Alexander, M. J., and K. H. Rosenlof, Nonstationary gravity wave forcing of the stratospheric zonal mean wind, J. Geophys. Res., 101, 23,465-23,474, 1996.

Alexander, M. J., and R. A. Vincent, Gravity waves in the 
tropical lower stratosphere: A model study of seasonal and interannual variability, J. Geophys. Res., 105, 22,299-22,310, 2000.

Alexander, M. J., J. R. Holton, and D. R. Durran, The gravity wave response above deep convection in a squall line simulation, J. Atmos. Sci., 52, 2212-2226, 1995.

Alexander, M. J., J. H. Beres, and L. Pfister, Tropical stratospheric gravity wave activity and relationship to clouds, $J$. Geophys. Res., 105, 22,299-22,309, 2000.

Alexander, M. J., T. Tsuda, and R. A. Vincent, Latitudinal variations observed in gravity waves with short vertical wavelengths, J. Atmos. Sci., 59, 1394-1404, 2002.

Allen, K. R., and R. I. Joseph, A canonical statistical theory of oceanic internal waves, J. Fluid Mech., 204, 185-228, 1989.

Allen, S. J., and R. A. Vincent, Gravity wave activity in the lower atmosphere: Seasonal and latitudinal variations, $J$. Geophys. Res, 100, 1327-1350, 1995.

Andreassen, Ø., C.-E. Wasberg, D. C. Fritts, and J. R. Isler, Gravity wave breaking in two and three dimensions, 1, Model description and comparison of two-dimensional evolutions, J. Geophys. Res., 99, 8095-8108, 1994.

Andreassen, Ø., P. Ø. Hvidsten, D. C. Fritts, and S. Arendt, Vorticity dynamics in a breaking gravity wave, 1, Initial instability evolution, J. Fluid Mech., 367, 27-46, 1998.

Andrews, D. G., and M. E. McIntyre, Planetary waves in horizontal and vertical shear: The generalized EliassenPalm relation and the mean zonal acceleration, J. Atmos. Sci., 33, 2031-2048, 1976.

Arendt, S., D. C. Fritts, and $\varnothing$. Andreassen, The initial value problem for Kelvin vortex waves, J. Fluid Mech., 344, 181212, 1997.

Arendt, S., D. C. Fritts, and $\varnothing$. Andreassen, Kelvin twist waves in the transition to turbulence, Eur. J. Mech., 17, 595-604, 1998.

Bacmeister, J. T., Mountain-wave drag in the stratosphere and mesosphere inferred from observed winds and a simple mountain-wave parameterization scheme, J. Atmos. Sci., 50, 377-399, 1993.

Bacmeister, J. T., S. D. Eckermann, P. A. Newman, L. Lait, K. R. Chan, M. Loewenstein, M. H. Proffitt, and B. L. Gary, Stratospheric horizontal wavenumber spectra of winds, potential temperature, and atmospheric tracers observed by high-altitude aircraft, J. Geophys. Res., 101, 9441-9470, 1996.

Bacmeister, J. T., M. R. Schoeberl, L. R. Lait, P. A. Newman, and B. L. Gary, Small-scale waves encountered during AASE, Geophys. Res. Lett., 17, 349-352, 1990a.

Bacmeister, J. T., M. R. Schoeberl, L. R. Lait, P. A. Newman, and B. L. Gary, ER-2 mountain wave encounter over Antarctica: Evidence for blocking, Geophys. Res. Lett., 17, 8184, 1990b.

Baldwin, M. P., et al., The quasi-biennial oscillation, Rev. Geophys., 39, 179-229, 2001.

Balsley, B. B., and D. A. Carter, The spectrum of atmospheric velocity fluctuations at 8 and $86 \mathrm{~km}$, Geophys. Res. Lett., 9, 465-468, 1982.

Balsley, B. B., and R. Garello, The kinetic energy density in the troposphere, stratosphere and mesosphere: A preliminary study using the Poker Flat radar in Alaska, Radio Sci., 20, 1355-1362, 1985.

Balsley, B. B., R. F. Woodman, M. Sarango, R. Rodriguez, J. Urbina, E. Ragaini, J. Carey, M. Huaman, and A. Giraldez, On the lack of Southern Hemisphere polar mesosphere summer echoes, J. Geophys. Res., 100, 11,685-11,693, 1995.

Barat, J., and C. Cot, Wind shear rotary spectra in the atmosphere, Geophys. Res. Lett., 19, 103-106, 1992.

Bergman, J. W., and M. L. Salby, Equatorial wave activity derived from fluctuations in observed convection, J. Atmos. Sci., 51, 3791-3806, 1994.
Blix, T. A., E. V. Thrane, D. C. Fritts, U. von Zahn, F.-J. Lübken, W. Hillert, S. P. Blood, J. D. Mitchell, G. A. Kokin, and S. V. Pakhomov, Small-scale structure observed in-situ during MAC/EPSILON, J. Atmos. Terres. Phys., 52, 835854, 1990.

Boville, B. A., and W. J. Randel, Equatorial waves in a strospheric GCM: Effects of vertical resolution, J. Atmos. Sci., 49, 785-801, 1992.

Broad, A. S., Linear theory of momentum fluxes in 3-D flows with turning of the mean wind with height, Q.J. R. Meteorol. Soc., 121, 1891-1902, 1995.

Broad, A. S., Do orographic gravity waves break in flows with uniform wind direction turning with height?, Q. J. R. Meteorol. Soc., 125, 1695-1714, 1999.

Broutman, D., On internal wave caustics, J. Phys. Oceanogr., 16, 1625-1635, 1986.

Broutman, D., and R. Grimshaw, The energetics of the interaction between short small-amplitude internal waves and inertial waves, J. Fluid Mech, 196, 93-106, 1988.

Broutman, D., and W. R. Young, On the interaction of smallscale internal waves with near-inertial waves, J. Fluid Mech., 166, 341-358, 1986.

Broutman, D., C. MaCaskill, M. E. McIntyre, and J. W. Rottman, On Doppler-spreading models of internal waves, Geophys. Res. Lett., 24, 2813-2816, 1997.

Bruhwiler, D. L., and T. J. Kaper, Wavenumber transport: Scattering of small-scale internal waves by large-scale wavepackets, J. Fluid Mech., 289, 379-405, 1995.

Buckley, G., D. Broutman, J. W. Rottman, and S. Eckermann, On the importance of weak steady shear in the refraction of short internal waves, Geophys. Res. Lett., 26, 2877-2880, 1999.

Bühler, O., and M. E. McIntyre, On shear-generated gravity waves that reach the mesosphere, part II, Wave propagation, J. Atmos. Sci., 56, 3764-3773, 1999.

Bühler, O., M. E. McIntyre, and J. F. Scinocca, On sheargenerated gravity waves that reach the mesosphere, part I, Wave generation, J. Atmos. Sci., 56, 3749-3763, 1999.

Cadet, D., and H. Teitelbaum, Observational evidence of internal inertia-gravity waves in the tropical stratosphere, $J$. Atmos. Sci., 36, 892-907, 1979.

Cadot, O., S. Douady, and Y. Couder, Characterization of the low-pressure filaments in a three-dimensional turbulent shear flow, Phys. Fluids, 7, 630-646, 1995.

Caulfield, C. P., and W. R. Peltier, Three dimensionalization of the stratified mixing layer, Phys. Fluids, 6, 3803-3805, 1994.

Chan, K. R., L. Pfister, T. P. Buil, S. W. Bowen, J. Dean-Day, B. L. Gary, D. W. Fahey, K. K. Kelly, C. R. Webster, and R. D. May, A case study of the mountain lee wave event of January 6, 1992, Geophys. Res. Lett., 20, 2551-2554, 1993.

Chao, W. C., and M. R. Schoeberl, On the linear approximation of gravity wave saturation in the mesosphere, J. Atmos. Sci., 41, 1893-1898, 1984.

Chimonas, G., and J. R. Grant, Shear excitation of gravity waves, part II, Upscale scattering from Kelvin-Helmholtz waves, J. Atmos. Sci., 41, 2278-2288, 1984.

Chimonas, G., and C. O. Hines, Doppler ducting of atmospheric gravity waves, J. Geophys. Res., 91, 1219-1230, 1986.

Chun, H.-Y., and J.-J. Baik, Momentum flux by thermally induced internal gravity waves and its approximation for large-scale models, J. Atmos. Sci., 55, 3299-3310, 1998.

Chunchuzov, I. P., On the high-wavenumber form of the Eulerian internal wave spectrum in the atmosphere, J. Atmos. Sci., 59, 1753-1774, 2002.

Clark, T. L., T. Hauf, and J. P. Kuettner, Convectively forced internal gravity waves: Results from two-dimensional numerical experiments, Q. J. R. Meteorol. Soc., 112, 899-925, 1986.

Collins, R. L., A. Nomura, and C. S. Gardner, Gravity waves in 
the upper mesosphere over Antarctica: Lidar observations at the South Pole and Syowa, J. Geophys. Res., 99, 54755485, 1994.

Cot, C. E., and J. Barat, Wave-turbulence interaction in the stratosphere: A case study, J. Geophys. Res., 91, 2749-2756, 1986.

Cot, C. E., and J. Barat, A "universal" wave spectrum for atmospheric temperature and velocity fluctuations in the stratosphere?, Geophys. Res. Lett., 17, 1577-1580, 1990.

Coulman, C. E., J. Vernin, and A. Fuchs, Optical seeingMechanism of formation of thin turbulent laminae in the atmosphere, Appl. Opt., 34, 5461-5474, 1995.

Coy, L., and D. C. Fritts, Gravity wave heat fluxes: A Lagrangian approach, J. Atmos. Sci., 45, 1770-1780, 1988.

Davis, P. A., and W. R. Peltier, Some characteristics of the Kelvin-Helmholtz and resonant overreflection modes of shear instability and of their interaction through vortex pairing, J. Atmos. Sci., 36, 2394-2412, 1979.

de la Torre, A., P. Alexander, and A. Giraldez, The kinetic to potential energy ratio and spectral separability from highresolution balloon soundings near the Andes mountains, Geophys. Res. Lett., 26, 1413-1416, 1999.

de la Torre, A., A. Giraldez, and P. Alexander, Saturated gravity wave spectra measured with balloons in Mendoza (Argentina), Geophys. Res. Lett., 21, 2039-2042, 1994.

de la Torre, A., H. Teitelbaum, and F. Vial, Stratospheric and tropospheric wave measurements near the Andes mountains, J. Atmos. Terres. Phys., 58, 521-530, 1996.

Dewan, E. M., Stratospheric wave spectra resembling turbulence, Science, 204, 832-835, 1979.

Dewan, E. M., Similitude modeling of internal gravity wave spectra, Geophys. Res. Lett., 18, 1473-1476, 1991.

Dewan, E. M., The saturated-cascade model for atmospheric gravity wave spectra, and the wavelength-period relations, Geophys. Res. Lett., 21, 817-820, 1994.

Dewan, E. M., Saturated-cascade similitude theory of gravity wave spectra, J. Geophys. Res., 102, 29,799-29,817, 1997.

Dewan, E. M., and R. E. Good, Saturation and the "universal" spectrum for vertical profiles of horizontal scalar winds in the atmosphere, J. Geophys. Res., 91, 2742-2748, 1986.

Dewan, E. M., N. Grossbard, A. F. Quesada, and R. E. Good, Spectral analysis of $10 \mathrm{~m}$ resolution scalar velocity profiles in the stratosphere, Geophys. Res. Lett., 11, 80-83, 1984.

Dewan, E. M., R. H. Picard, R. R. O’Neil, H. A. Gardiner, J. Gibson, J. D. Mill, E. Richards, M. Kendra, and W. O. Gallery, MSX satellite observations of thunderstorm-generated gravity waves in mid-wave infrared images of the upper stratosphere, Geophys. Res. Lett., 25, 939-942, 1998.

Dong, B., and K. C. Yeh, Resonant and nonresonant wavewave interactions in an isothermal atmosphere, J. Geophys. Res., 93, 3729-3744, 1988.

Dörnbrack, A., M. Leutbecher, R. Kivi, and E. Kyrö, Mountainwave induced record low stratospheric temperatures above northern Scandinavia, Tellus, Ser. A, 51, 951-963, 1999.

Dunkerton, T. J., Stochastic parameterization of gravity wave stresses, J. Atmos. Sci., 39, 1711-1725, 1982a.

Dunkerton, T. J., Theory of the mesopause semiannual oscillation, J. Atmos. Sci., 39, 2681-2690, 1982b.

Dunkerton, T. J., Inertia-gravity waves in the stratosphere, $J$. Atmos. Sci., 41, 3396-3404, 1984.

Dunkerton, T. J., Effect of nonlinear instability on gravity wave momentum Transport, J. Atmos. Sci., 44, 3188-3209, 1987.

Dunkerton, T. J., Theory of internal gravity wave saturation, Pure Appl. Geophys., 130, 373-397, 1989.

Dunkerton, T. J., Shear instability of internal inertia-gravity waves, J. Atmos. Sci., 54, 1628-1641, 1997a.

Dunkerton, T. J., The role of gravity waves in the quasibiennial oscillation, J. Geophys. Res., 102, 26,053-26,076, 1997b.
Durran, D. R., and J. B. Klemp, Another look at downslope winds, part II, Nonlinear amplification beneath wave overturning layers, J. Atmos. Sci., 44, 3402-3412, 1987.

Eckermann, S. D., Effects of nonstationarity on spectral analysis of mesoscale motions in the atmosphere, J. Geophys. Res., 95, 16,685-16,703, 1990.

Eckermann, S. D., Ray-tracing simulation of the global propagation of inertia gravity waves through the zonally averaged middle atmosphere, J. Geophys. Res., 97, 15,84915,866, 1992.

Eckermann, S. D., Effect of background winds on vertical wavenumber spectra of atmospheric gravity waves, J. Geophys. Res., 100, 14,097-14,112, 1995a.

Eckermann, S. D., On the observed morphology of gravitywave and equatorial-wave variance in the stratosphere, $J$. Atmos. Terr. Phys., 57, 105-134, 1995b.

Eckermann, S. D., Influence of wave propagation on the Doppler-spreading of atmospheric gravity waves, J. Atmos. Sci., 54, 2554-2573, 1997.

Eckermann, S. D., Isentropic advection by gravity waves: Quasi-universal $M^{-3}$ vertical wavenumber spectra near the onset of instability, Geophys. Res. Lett., 26, 201-204, 1999.

Eckermann, S. D., and W. K. Hocking, Effect of superposition on measurements of atmospheric gravity waves: A cautionary note and some reinterpretations, J. Geophys. Res., 94, 6333-6339, 1989.

Eckermann, S. D., and P. Preusse, Global measurements of stratospheric mountain waves from space, Science, 286, 1534-1537, 1999.

Eckermann, S. D., and R. A. Vincent, Falling sphere observations of anisotropic gravity wave motions in the upper stratosphere over Australia, Pure Appl. Geophys., 130, 509532, 1989.

Eckermann, S. D., and R. A. Vincent, ST radar observations of gravity-wave production by cold fronts over southern Australia, J. Atmos. Sci., 50, 785-806, 1993.

Eckermann, S. D., I. Hirota, and W. K. Hocking, Gravity wave and equatorial wave morphology of the stratosphere derived from long-term rocket soundings, $Q . J$. R. Meteorol. Soc., 121, 149-186, 1995.

Edmon, H. J., Jr., B. J. Hoskins, and M. E. McIntyre, EliassenPalm cross sections for the troposphere, J. Atmos. Sci., 37, 2600-2616, 1980.

Fan, Y., J. Klostermeyer, and R. Rüster, VHF radar observation of gravity wave critical levels in the mid-latitude summer mesopause region, Geophys. Res. Lett., 18, 697-700, 1991.

Farmer, D., and L. Armi, Stratified flow over topography: The role of small-scale entrainment and mixing in flow establishment, Proc. R. Soc. London, Ser. A, 455, 3221-3258, 1999.

Farrell, B. F., and P. J. Ioannou, Generalized stability theory, part I, Autonomous operators, J. Atmos. Sci., 53, 20252040, 1996a.

Farrell, B. F., and P. J. Ioannou, Generalized stability theory, part II, Nonautonomous operators, J. Atmos. Sci., 53, 20412053, 1996b.

Fels, S. B., The radiative damping of short vertical scale waves in the mesosphere, J. Atmos. Sci., 41, 1755-1764, 1984.

Fetzer, E. J., and J. C. Gille, Gravity wave variance in LIMS temperatures, part I, Variability and comparison with background winds, J. Atmos. Sci., 51, 2461-2483, 1994.

Fetzer, E. J., and J. C. Gille, Gravity wave variance in LIMS temperatures, part II, Comparison with the zonal-mean momentum balance, J. Atmos. Sci., 53, 398-410, 1996.

Fleming, E. L., S. Chandra, M. R. Schoeberl, and J. J. Barnett, Monthly mean global climatology of temperature, wind, geopotential height, and pressure for 0-120 km, NASA Tech. Memo., TM-100697, 85 pp., 1988. 
Forbes, J. M., J. Gu, and S. Miyahara, On the interactions between gravity waves and the diurnal propagating tide, Planet. Space Sci., 39, 1249-1257, 1991.

Fovell, R., D. Durran, and J. R. Holton, Numerical simulations of convectively generated stratospheric gravity waves, $J$. Atmos. Sci., 49, 1427-1442, 1992.

Fritts, D. C., Gravity wave saturation in the middle atmosphere: A review of theory and observations, Rev. Geophys., 22, 275-308, 1984a.

Fritts, D. C., Shear excitation of atmospheric gravity waves, part II, Nonlinear radiation from a free shear layer, $J$. Atmos. Sci., 41, 524-537, 1984b.

Fritts, D. C., A review of gravity wave saturation processes, effects, and variability in the middle atmosphere, Pure Appl. Geophys., 130, 343-371, 1989.

Fritts, D. C., Errant inferences of gravity wave momentum and heat fluxes using airglow and lidar instrumentation: Corrections and cautions, J. Geophys. Res., 105, 22,355-22,360, 2000.

Fritts, D. C., and H.-G. Chou, An investigation of the vertical wavenumber and frequency spectra of gravity wave motions in the lower stratosphere, J. Atmos. Sci., 44, 3610-3624, 1987.

Fritts, D. C., and T. J. Dunkerton, A quasi-linear study of gravity wave saturation and self-acceleration, J. Atmos. Sci., 41, 3272-3288, 1984.

Fritts, D. C., and T. J. Dunkerton, Fluxes of heat and constituents due to convectively unstable gravity waves, J. Atmos. Sci., 42, 549-556, 1985.

Fritts, D. C., and M. A. Geller, Viscous stabilization of gravity wave critical level flows, J. Atmos. Sci., 33, 2276-2284, 1976.

Fritts, D. C., and U.-P. Hoppe, High-resolution measurements of vertical velocity with the EISCAT VHF radar, 2, Spectral observations and model comparisons, J. Geophys. Res., 100, 16,827-16,838, 1995.

Fritts, D. C., and W. Lu, Spectral estimates of gravity wave energy and momentum fluxes, II, Parameterization of wave forcing and variability, J. Atmos. Sci., 50, 3695-3713, 1993.

Fritts, D. C., and Z. Luo, Gravity wave excitation by geostrophic adjustment of the jet stream, part I, Two-dimensional forcing, J. Atmos. Sci., 49, 681-697, 1992.

Fritts, D. C., and Z. Luo, Dynamical and radiative forcing of the summer mesopause circulation and thermal structure, 1, Mean solstice conditions, J. Geophys. Res., 100, 31193128, 1995.

Fritts, D. C., and G. D. Nastrom, Sources of mesoscale variability of gravity waves, II, Frontal, convective, and jet stream excitation, J. Atmos. Sci., 49, 111-127, 1992.

Fritts, D. C., and P. K. Rastogi, Convective and dynamical instabilities due to gravity wave motions in the lower and middle atmosphere: Theory and observations, Radio Sci., 20, 1247-1277, 1985.

Fritts, D. C., and T. E. VanZandt, Effects of Doppler shifting on the frequency spectra of atmospheric gravity waves, $J$. Geophys. Res., 92, 9723-9732, 1987.

Fritts, D. C., and T. E. VanZandt, Spectral estimates of gravity wave energy and momentum fluxes, I, Energy dissipation, acceleration, and constraints, J. Atmos. Sci., 50, 3685-3694, 1993.

Fritts, D. C., and R. A. Vincent, Mesospheric momentum flux studies at Adelaide, Australia: Observations and a gravity wave/tidal interaction model, J. Atmos. Sci., 44, 605-619, 1987.

Fritts, D. C., and D.-Y. Wang, Doppler-shifting effects on frequency spectra of gravity waves observed near the summer mesopause at high latitude, J. Atmos. Sci., 48, 15351544, 1991.

Fritts, D. C., and J. A. Werne, Turbulence dynamics and mixing due to gravity waves in the lower and middle atmo- sphere, in Atmospheric Science Across the Stratopause, Geophys. Monogr. Ser., vol. 123, edited by D. E. Siskind, S. D. Eckermann, and M. E. Summers, pp. 143-159, AGU, Washington, D. C., 2000.

Fritts, D. C., and L. Yuan, An analysis of gravity wave ducting in the atmosphere: Eckarts resonances in thermal and Doppler ducts, J. Geophys. Res., 94, 18,455-18,466, 1989a.

Fritts, D. C., and L. Yuan, Measurement of momentum fluxes near the summer mesopause at Poker Flat, Alaska, J. Atmos. Sci., 46, 2569-2579, 1989b.

Fritts, D. C., and L. Yuan, Stability analysis of inertio-gravity wave structure in the middle atmosphere, J. Atmos. Sci., 46, $1738-1745,1989 \mathrm{c}$.

Fritts, D. C., T. Tsuda, T. Sato, S. Fukao, and S. Kato, Observational evidence of a saturated gravity wave spectrum in the troposphere and lower stratosphere, J. Atmos. Sci., 45, 1741-1759, 1988a.

Fritts, D. C., S. A. Smith, B. B. Balsley, and C. R. Philbrick, Evidence of gravity wave saturation and local turbulence production in the summer mesosphere and lower thermosphere during the STATE experiment, J. Geophys. Res., 93, 7015-7025, 1988b.

Fritts, D. C., R. C. Blanchard, and L. Coy, Gravity wave structure between 60 and $90 \mathrm{~km}$ inferred from space shuttle reentry data, J. Atmos. Sci., 46, 423-434, 1989.

Fritts, D. C., U.-P. Hoppe, and B. Inhester, A study of the vertical motion field near the high-latitude summer mesopause during MAC/SINE, J. Atmos. Terr. Phys., 52, 927-938, 1990a.

Fritts, D. C., T. Tsuda, T. E. VanZandt, S. A. Smith, T. Sato, S. Fukao, and S. Kato, Studies of velocity fluctuations in the lower atmosphere using the MU radar, II, Momentum fluxes and energy densities, J. Atmos. Sci., 47, 51-66, $1990 \mathrm{~b}$.

Fritts, D. C., L. Yuan, M. H. Hitchman, L. Coy, E. Kudeki, and R. F. Woodman, Dynamics of the equatorial mesosphere observed using the Jicamarca MST radar during June and August 1987, J. Atmos. Sci., 49, 2353-2371, 1992.

Fritts, D. C., D.-Y. Wang, R. C. Blanchard, Gravity wave and tidal structures between 60 and $140 \mathrm{~km}$ inferred from space shuttle re-entry data, J. Atmos. Sci., 50, 785-806, 1993a.

Fritts, D. C., J. R. Isler, G. E. Thomas, and $\varnothing$. Andreassen, Wave breaking signatures in noctilucent clouds, Geophys. Res. Lett., 20, 2039-2042, 1993 b.

Fritts, D. C., J. R. Isler, and Ø. Andreassen, Gravity wave breaking in two and three dimensions, 2, Three-dimensional evolution and instability structure, J. Geophys. Res., 99, 8109-8123, 1994.

Fritts, D. C., J. F. Garten, and Ø. Andreassen, Wave breaking and transition to turbulence in stratified shear flows, $J$. Atmos. Sci., 53, 1057-1085, 1996a.

Fritts, D. C., T. L. Palmer, Ø. Andreassen, and I. Lie, Evolution and breakdown of Kelvin-Helmholtz billows in stratified compressible flows, I, Comparison of two- and threedimensional flows, J. Atmos. Sci., 53, 3173-3191, 1996 b.

Fritts, D. C., et al., Equatorial dynamics observed by rocket, radar, and satellite during the CADRE/MALTED campaign, 2, Mean and wave structures, coherence, and variability, J. Geophys. Res., 102, 26,11-26,216, 1997a.

Fritts, D. C., J. R. Isler, J. H. Hecht, R. L. Walterscheid, Ø. Andreassen, Wave breaking signatures in sodium densities and $\mathrm{OH}$ nightglow, part II, Simulation of wave and instability structures, J. Geophys. Res., 102, 6669-6684, 1997b.

Fritts, D. C., S. Arendt, and Ø. Andreassen, Vorticity dynamics in a breaking internal gravity wave, 2, Vortex interactions and transition to turbulence, J. Fluid Mech., 367, 47-65, 1998.

Fritts, D. C., S. Arendt, and $\varnothing$. Andreassen, The vorticity dynamics of instability and turbulence in a breaking internal gravity wave, Earth Planets Space, 51, 457-473, 1999. 
Fritts, D. C., S. L. Vadas, and Y. Yamada, An estimate of strong local body forcing and gravity wave radiation based on $\mathrm{OH}$ airglow and meteor radar observations, Geophys. Res. Lett., 29(10), 1429, doi:10.1029/2001GL013753, 2002.

Fritts, D. C., C. Bizon, J. A. Werne, and C. K. Meyer, Layering accompanying turbulence generation due to shear instability and gravity wave breaking, J. Geophys. Res., 108, doi: 10.1029/2002JD002406, in press 2003.

Fukao, S., Y. Maekawa, T. Sato, and S. Kato, Fine structure in mesospheric wind fluctuations observed by the Arecibo UHF Doppler radar, J. Geophys. Res., 90, 7547-7556, 1985.

Fuller-Rowell, T. J., The impact of gravity waves on nitric oxide in the lower Thermosphere, J. Atmos. Terr. Phys., 56, 1387-1398, 1994.

Gage, K. S., Evidence for $\mathrm{k}^{-5 / 3}$ law inertial range in mesoscale two-dimensional turbulence, J. Atmos. Sci., 36, 1950-1954, 1979.

Garcia, R. R., Dynamics, radiation, and photochemistry in the mesosphere: Implications for the formation of noctilucent clouds, J. Geophys. Res., 94, 14,605-14,615, 1989.

Garcia, R. R., and B. Boville, "Downward control" of the mean meridional circulation and temperature distribution of the polar winter stratosphere, J. Atmos. Sci., 51, 2238-2245, 1994.

Garcia, R. R., and F. Sassi, Modulation of the Mesospheric Semiannual Oscillation by the Quasibiennial Oscillation, Earth Planets Space, 51, 563-569, 1999.

Garcia, R. R., and S. Solomon, The effect of breaking gravity waves on the dynamics and chemical composition of the mesosphere and lower thermosphere, J. Geophys. Res., 90, 3850-3868, 1985.

Garcia, R. R., T. I. Dunkerton, R. S. Lieberman, and R. A. Vincent, Climatology of the semiannual oscillation of the tropical middle atmosphere, J. Geophys. Res., 102, 26,01926,032, 1997.

Gardner, C. S., Diffusive filtering theory of gravity wave spectra in the atmosphere, J. Geophys. Res., 99, 20,601-20,622, 1994.

Gardner, C. S., C. A. Hostetler, and S. J. Franke, Gravity wave models for the horizontal wave number spectra of atmospheric velocity and density fluctuations, J. Geophys. Res., 98, 1035-1049, 1993.

Gardner, C. S., M. Coble, G. C. Papen, and G. S. Swenson, Observations of the unambiguous two-dimensional horizontal wave number spectrum of $\mathrm{OH}$ intensity perturbations, Geophys. Res. Lett., 23, 3739-3742, 1996.

Gardner, C. S., K. Gulati, Y. Zhao, and G. Swenson, Measuring gravity wave momentum fluxes with airglow imagers, $J$. Geophys. Res., 104, 11,903-11,915, 1999a.

Gardner, C. S., S. J. Franke, W. Yang, X. Tao, and J. R. Yu, Interpretation of gravity waves observed in the mesopause region at Starfire Optical Range, New Mexico: Strong evidence for nonseparable intrinsic (m, omega) spectra, $J$. Geophys. Res., 103, 8699-8713, 1999 b.

Gary, B. L., Observational results using the microwave temperature profiler during the airborne antarctic ozone experiment, J. Geophys. Res., 94, 11,223-11,231, 1989.

Gavrilov, N. M., and V. A. Yudin, Model for coefficients of turbulence and effective Prandtl number produced by breaking gravity waves in the upper atmosphere, J. Geophys. Res., 97, 7619-7624, 1992.

Gavrilov, N. M., S. Fukao, T. Nakamura, T. Tsuda, M. D. Yamanaka, and M. Yamamoto, Statistical analysis of gravity waves observed with the middle and upper atmosphere radar in the middle atmosphere, 1, Method and general characteristics, J. Geophys. Res., 101, 29,511-29,521, 1996.

Gerz, T., J. Howell, and L. Mahrt, Vortex structures and microfronts, Phys. Fluids, 6, 1242-1251, 1994.
Gill, A. E., Atmosphere-Ocean Dynamics, 662 pp., Academic, San Diego, Calif., 1982.

Gille, J. C., and J. M. Russell III, The limb infrared monitor of the stratosphere: Experiment description, performance, and results, J. Geophys. Res., 89, 5125-5140, 1984.

Goldberg, R. A., G. Lehmacher, F. J. Schmidlin, D. C. Fritts, J. D. Mitchell, C. L. Croskey, M. Friedrich, and W. E. Swartz, Equatorial dynamics observed by rocket, radar, and satellite during the CADRE/MALTED campaign, 1, Programmatics and small-scale fluctuations. J. Geophys. Res., 102, 26,179-26,190, 1997.

Gray, L. J., and J. A. Pyle, A two-dimensional model of the quasi-biennial oscillation of ozone, J. Atmos. Sci., 46, 203220, 1989.

Griffiths, M., and M. J. Reeder, Stratospheric inertia-gravity waves generated in a numerical model of frontogenesis, I, Model solutions, Q. J. R. Meteorol. Soc., 122, 1153-1174, 1996.

Grimshaw, R. H. J., Resonant wave interactions in a stratified shear flow, J. Fluid Mech., 190, 357-374, 1988.

Guest, F. M., M. J. Reeder, C. J. Marks, and D. J. Karoly, Inertia-gravity waves observed in the lower stratosphere over Macquarie Island, J. Atmos. Sci., 57, 737-752, 2000.

Hall, C. M., and U.-P. Hoppe, Estimates of turbulent energy dissipation rates from determinations of characteristic vertical wavenumber by EISCAT, Geophys. Res. Lett., 25, 4075-4078, 1998.

Hamilton, K., Diagnostic study of the momentum balance in the Northern Hemisphere winter stratosphere, Mon. Weather Rev., 111, 1434-1441, 1983.

Hamilton, K., Climatological statistics of stratospheric intertiagravity waves deduced from historical rocketsonde wind and temperature data, J. Geophys. Res., 96, 20,831-20,839, 1991.

Hamilton, K., Comprehensive simulation of the middle atmospheric climate: Some recent results, Clim. Dyn., 11, 223241, 1995.

Hamilton, K., Comprehensive meteorological modelling of the middle atmosphere: A tutorial review, J. Atmos. Terr. Phys., 58, 1591-1627, 1996.

Hamilton, K. (Ed.), Gravity Wave Processes and Their Parameterization in Global Climate Models, 404 pp., SpringerVerlag, New York, 1997.

Hamilton, K., and R. A. Vincent, High-resolution radiosonde data offer new prospects for research, Eos Trans. AGU, 76, 497, 1995.

Hamilton, K., R. J. Wilson, J. D. Mahlman, and L. J. Umscheid, Climatology of the SKYHI troposphere stratosphere mesosphere general circulation model, J. Atmos. Sci., 52, 5-43, 1995.

Hamilton, K., R. J. Wilson, and R. S. Hemler, Middle atmosphere simulated with high vertical and horizontal resolution versions of a GCM: Improvements in the cold pole bias and generation of a QBO-like oscillation in the tropics, $J$. Atmos. Sci., 56, 3829-3846, 1999.

Haque, R., and G. R. Swenson, Extraction of motion parameters of gravity-wave structures from all-sky $\mathrm{OH}$ image sequences, Appl. Opt., 38, 4433-4442, 1999.

Hauchecorne, A., M. L. Chanin, and R. Wilson, Mesopheric temperature inversion and gravity wave breaking, Geophys. Res. Lett., 14, 933-936, 1987.

Haynes, P. H., C. J. Marks, M. E. McIntyre, T. G. Shephard, and K. P. Shine, On the "downward control" of extratropical diabatic circulations by eddy-induced mean zonal forces, J. Atmos. Sci., 48, 657-678, 1991.

Hecht, J. H., R. L. Walterscheid, D. C. Fritts, J. R. Isler, D. C. Senft, C. S. Gardner, and S. J. Franke, Wave breaking signatures in $\mathrm{OH}$ airglow and sodium densities and temperatures, part I, Airglow imaging, Na lidar, and MF radar 
observations, J. Geophys. Res., 102, 6655-6668, 1997.

Hecht, J. H., C. Fricke-Bergemann, and R. L. Walterscheid, Observations of the breakdown of an atmospheric gravity wave near the cold summer mesopause at 54N, Geophys. Res. Lett., 27, 879-882, 2000.

Hertzog, A., and F. Vial, A study of the dynamics of the equatorial lower atmosphere by use of ultra-long duration balloons, 2, Gravity waves, J. Geophys. Res., 106, 22,74522,761, 2001.

Hickey, M. P., M. J. Taylor, C. S. Gardner, and C. R. Gibbons, Full-wave modeling of small-scale gravity waves using Airborne Lidar and Observations of the Hawaiian Airglow (ALOHA-93) O(1S) images and coincident $\mathrm{Na}$ wind/temperature lidar Measurements, J. Geophys. Res., 103, 64396453, 1998.

Hines, C. O., Internal atmospheric gravity waves at ionospheric heights, Can. J. Phys., 38, 1441-1481, 1960.

Hines, C. O., Generalizations of the Richardson criterion for the onset of atmospheric turbulence, Q. J. R. Meteorol. Soc, 97, 429-439, 1971.

Hines, C. O., A modeling of atmospheric gravity waves and wave drag generated by isotropic and anisotropic terrain, $J$. Atmos. Sci, 45, 309-322, 1988a.

Hines, C. O., The generation of turbulence by atmospheric gravity waves, J. Atmos. Sci., 45, 1269-1278, 1988b.

Hines, C. O., The saturation of gravity waves in the middle atmosphere, part I, Critique of linear-instability theory, $J$. Atmos. Sci., 48, 1348-1359, 1991.

Hines, C. O., The saturation of gravity waves in the middle atmosphere, part IV, Cutoff of the incident wave spectrum, J. Atmos. Sci., 50, 3045-3060, 1993.

Hines, C. O., Nonlinearity of gravity wave saturated spectra in the middle atmosphere, Geophys. Res. Lett., 23, 3309-3312, 1996.

Hines, C. O., Doppler-spread parameterization of gravity-wave momentum deposition in the middle atmosphere, 1, Basic formulation, J. Atmos. Sol. Terr. Phys., 59, 371-386, 1997 a.

Hines, C. O., Doppler-spread parameterization of gravity-wave momentum deposition in the middle atmosphere, 2, Broad and quasi monochromatic spectra, and implementation, $J$. Atmos. Sol. Terr. Phys., 59, 387-400, 1997b.

Hines, C. O., Comments on paper by S.D. Eckermann, "Influence of wave propagation on the Doppler spreading of atmospheric gravity waves," J. Atmos. Sci., 56, 1094-1098, 1999.

Hines, C. O., Theory of the Eulerian tail in the spectra of atmospheric and oceanic internal gravity waves, J. Fluid Mech., 448, 289-313, 2001.

Hines, C. O., Nonlinearities and linearities in internal gravity waves of the atmosphere and oceans, Geophys. Astrophys. Fluid Dyn., 96, 1-30, 2002a.

Hines, C. O., Comments on the gravity wave theory of J. Weinstock concerning dissipation induced by nonlinear effects, J. Atmos. Sci., 59, 2024-2030, 2002b.

Hirota, I., Climatology of gravity waves in the middle atmosphere, J. Atmos. Terr. Phys., 46, 767-773, 1984.

Hirota, I., and T. Niki, A statistical study of inertia-gravity waves in the middle atmosphere, J. Meteorol. Soc. Jpn., 63, 1055-1066, 1985.

Hitchman, M. H., J. C. Gille, C. D. Rodgers, and G. Brasseur, The separated polar winter stratopause: A gravity wave driven climatological feature, J. Atmos. Sci., 46, 410-422, 1989.

Hitchman, M. H., K. W. Bywaters, D. C. Fritts, L. Coy, E. Kudeki, and F. Surucu, Mean winds and momentum fluxes over Jicamarca, Peru, during June and August 1987, J. Atmos. Sci., 49, 2372-2383, 1992.

Hitchman, M. H., and C. B. Leovy, Estimation of the Kelvin wave contribution to the semiannual oscillation, J. Atmos. Sci., 45, 1462-1475, 1988.
Hocking, W. K., Turbulence in the region $80-120 \mathrm{~km}, A d v$. Space Res., 10, 153-161, 1990.

Hocking, W. K., S. Fukao, M. Yamamoto, T. Tsuda, and S. Kato, Viscosity waves and thermal conduction waves as a cause of "specular" reflectors in radar studies of the atmosphere, Radio Sci., 26, 1281-1303, 1991.

Hodges, R. R., Jr., Generation of turbulence in the upper atmosphere by internal gravity waves, J. Geophys. Res., 72, 3455-3458, 1967.

Holton, J. R., The role of gravity wave induced drag and diffusion in the momentum budget of the mesosphere, $J$. Atmos. Sci., 39, 791-799, 1982.

Holton, J. R., The influence of gravity wave breaking on the general circulation of the middle atmosphere, J. Atmos. Sci., 40, 2497-2507, 1983.

Holton, J. R., The generation of mesospheric planetary waves by zonally asymmetric gravity wave breaking, J. Atmos. Sci., 41, 3427-3430, 1984.

Holton, J. R., An Introduction to Dynamic Meteorology, 507 pp., Academic, San Diego, Calif., 1992.

Holton, J. R., and M. J. Alexander, The role of waves in the transport circulation of the middle atmosphere, in Atmospheric Science Across the Stratopause, Geophys. Monogr. Ser., vol. 123, edited by D. E. Siskind, S. D. Eckermann, and M. E. Summers, pp. 21-35, AGU, Washington, D. C., 2000.

Holton, J. R., and M. R. Schoeberl, The role of gravity wave generated advection and diffusion in transport of tracers in the mesosphere, J. Geophys. Res., 93, 11,075-11,082, 1988.

Holton, J. R., M. J. Alexander, and M. T. Boehm, Evidence for short vertical wavelength Kelvin waves in the DOE-ARM Nauru99 radiosonde data, J. Geophys. Res., 106, 20,12520,129, 2001.

Hoppe, U.-P., and D. C. Fritts, High-resolution measurements of vertical velocity with the EISCAT VHF radar, 1, Motion field characteristics and measurement biases, J. Geophys. Res., 100, 16,813-16,826, 1995.

Hoppe, U.-P., T. Eriksen, E. V. Thrane, T. A. Blix, J. Fiedler, and F.-J. Lübken, Observations in the polar middle atmosphere by rocket-borne Rayleigh lidar: First results, Earth Planets Space, 51, 815-824, 1999.

Horinouchi, T., and S. Yoden, Wave-mean flow interaction associated with a QBO-like oscillation simulated in a simplified GCM, J. Atmos. Sci., 55, 502-526, 1998.

Huaman, M. M., and B. B. Balsley, Differences in near-mesopause summer winds, temperatures, and water vapor at northern and southern latitudes as possible causal factors for inter-hemispheric PMSE differences, Geophys. Res. Lett., 26, 1529-1532, 1999.

Huang, T. Y. W., and A. K. Smith, The mesospheric diabatic circulation and the parameterized thermal effect of gravity wave breaking on the circulation, J. Atmos. Sci., 48, 10931111, 1991.

Huang, T. Y. W., and A. K. Smith, Dynamical and chemical feedback in a two-dimensional interactive model of the middle atmosphere, J. Geophys. Res., 100, 11,085-11,104, 1995.

Isler, J. R., and D. C. Fritts, Gravity wave variability and interaction with lower-frequency motions in the mesosphere and lower thermosphere over Hawaii, J. Atmos. Sci., 53, 37-48, 1996.

Isler, J. R., D. C. Fritts, and $\varnothing$. Andreassen, Gravity wave breaking in two and three dimensions, 3, Vortex breakdown and transition to turbulence, J. Geophys. Res., 99, 81258137, 1994.

Isler, J. R., M. J. Taylor, and D. C. Fritts, Observational evidence of wave ducting and evanescence in the mesosphere, J. Geophys. Res., 102, 26,301-26,313, 1997.

Jackson, D. R., and L. J. Gray, Simulation of the semi-annual oscillation of the equatorial middle atmosphere using the 
Extended UGAMP General Circulation Model, Q. J. R. Meteorol. Soc., 120, 1559-1588, 1994.

Jasperson, W. H., G. D. Nastrom, and D. C. Fritts, Further study of terrain effects on the mesoscale spectrum of atmospheric motions, J. Atmos. Sci., 47, 979-987, 1990.

Jeong, J., and F. Hussain, On the identification of a vortex, $J$. Fluid Mech., 285, 69-94, 1995.

Jones, P. W., K. Hamilton, and R. J. Wilson, A very high resolution general circulation model simulation of the global circulation in austral winter, J. Atmos. Sci., 54, 11071116, 1997.

Karoly, D. J., G. L. Roff, and M. J. Reeder, Gravity wave activity associated with tropical convection detected in TOGA COARE sounding data, Geophys. Res. Lett., 23, 261-264, 1996.

Kennedy, P. J., and M. A. Shapiro, Further encounters with clear air turbulence in research aircraft, J. Atmos. Sci., 37, 986-993, 1979.

Kershaw, R., Parameterization of momentum transport by convectively generated gravity waves, Q. J. R. Meteorol. Soc., 121, 1023-1040, 1995.

Kiehl, J. T., J. J. Hack, G. B. Bonan, B. A. Boville, B. P. Briegleb, D. L. Williamson, and P. J. Rasch, Description of the NCAR Community Climate Model (CCM3), NCAR Tech. Note 420, 96-103, Natl. Cent. for Atmos. Res., Boulder, Colo., 1996.

Kinnersley, J. S., The climatology of the stratospheric "THIN AIR" model, Q. J. R. Meteorol. Soc., 122, 219-252, 1996.

Kitamura, Y., and I. Hirota, Small-scale disturbances in the lower stratosphere revealed by daily rawin sonde observations, J. Meteorol. Soc. Jpn., 67, 817-830, 1989.

Klaassen, G. P., and W. R. Peltier, The onset of turbulence in finite-amplitude Kelvin-Helmholtz billows, J. Fluid Mech., 155, 1-35, 1985.

Klostermeyer, J., Observations indicating parametric instabilities of internal gravity waves at thermospheric heights, Geophys. Astrophys. Fluid Dyn., 29, 117-138, 1984.

Klostermeyer, J., On the role of parametric instability of internal gravity waves in atmospheric radar observations, Radio Sci., 25, 983-995, 1990.

Klostermeyer, J., Two- and three-dimensional parametric instabilities in finite amplitude internal gravity waves, Geophys. Astrophys. Fluid Dyn., 64, 1-25, 1991.

Kuettner, J. P., P. A. Hildebrand, and T. L. Clark, Convection waves: Observations of gravity wave systems over convectively active boundary layers, Q. J. R. Meteorol. Soc., 113, 445-467, 1987.

Kuo, F.-S., H.-W. Shen, I.-J. Fu, J.-K. Chao, J. Röttger, and C. H. Liu, Altitude dependence of vertical velocity spectra observed by VHF radar, Radio Sci., 20, 1349-1354, 1985.

Kuo, F. S., H. Y. Lue, C. M. Huang, C. L. Lo, C. H. Liu, and S. Fukao, A study of velocity fluctuation spectra in the troposphere and lower stratosphere using MU radar, $J$. Atmos. Terres. Phys., 54, 31-48, 1992.

Lalas, D. P., and F. Einaudi, On characteristics of gravity waves generated by atmospheric shear layers, J. Atmos. Sci., 33, 1248-1259, 1976.

Lane, T. P., M. J. Reeder, and T. L. Clark, Numerical modeling of gravity wave generation by deep tropical convection, $J$. Atmos. Sci., 58, 1249-1274, 2001.

Larsen, M. F., R. F. Woodman, T. Sato, and M. K. Davis, Power spectra of oblique velocities in the troposphere and lower stratosphere observed at Arecibo, Puerto Rico, J. Atmos. Sci., 43, 2230-2240, 1986.

Lawrence, B. N., The effect of parameterized gravity wave drag on simulations of the middle atmosphere during northern winter 1991/1992-General evolution, in Gravity Wave Processes: Their Parameterization in Global Climate Models,
NATO ASI Ser., Ser. I, vol. 50, edited by K. Hamilton, pp. 291-307, Springer-Verlag, New York, 1997.

Lefrere, J., and C. Sidi, Distortion of gravity wave spectra measured from ground-based stations, Geophys. Res. Lett., 17, 1581-1584, 1990.

LeLong, M.-P., and T. J. Dunkerton, Inertia-gravity wave breaking in three dimensions, 1 , Convectively stable waves, J. Atmos. Sci., 55, 2473-2488, 1998 a.

LeLong, M.-P., and T. J. Dunkerton, Inertia-gravity wave breaking in three dimensions, 2, Convectively unstable waves, J. Atmos. Sci., 55, 2489-2501, 1998b.

Leutbecher, M., and H. Volkert, Propagation of mountain waves into the stratosphere: Quantitative evaluation of three-dimensional simulations, J. Atmos. Sci., 57, 30903108, 2000.

Lieberman, R. S., et al., HRDI observations of mean meridional winds at solstice, J. Atmos. Sci., 55, 1887-1896, 1998.

Lighthill, J., Waves in Fluids, 504 pp., Cambridge Univ. Press, New York, 1978.

Lilly, D. K., Stratified turbulence and the mesoscale variability of the atmosphere, J. Atmos. Sci., 40, 749-761, 1983.

Lilly, D. K., and P. J. Kennedy, Observations of a stationary mountain wave and its associated momentum flux and energy dissipation, J. Atmos. Sci., 30, 1135-1152, 1973.

Lilly, D. K., and P. F. Lester, Waves and turbulence in the stratosphere, J. Atmos. Sci., 31, 800-812, 1974.

Lindzen, R. S., Wave-mean flow interactions in the upper atmosphere, Boundary Layer Meteorol., 4, 327-343, 1973.

Lindzen, R. S., Turbulence and stress owing to gravity wave and tidal breakdown, J. Geophys. Res., 86, 9707-9714, 1981.

Lindzen, R. S., and J. R. Holton, A theory of the quasi-biennial oscillation, J. Atmos. Sci., 25, 1095-1107, 1968.

Lintelman, S. A., and C. S. Gardner, Observation and interpretation of spectra of atmospheric gravity wave perturbations with upward and downward phase progression, $J$. Geophys. Res., 99, 16,959-16,971, 1994.

Liu, H.-L., M. E. Hagan, and R. G. Roble, Local mean state changes due to gravity wave breaking modulated by the diurnal tide, J. Geophys. Res., 105, 12,381-12,396, 2000.

Lombard, P. N., and J. J. Riley, Instability and breakdown of internal gravity Waves, I, Linear stability analysis, Phys. Fluids, 8, 3271-3287, 1996.

Long, R. R., Some aspects of the flow of stratified fluids, III, Continuous density gradients, Tellus, 7, 341-357, 1955.

Lott, F., Linear mountain drag and averaged pseudo-momentum flux profiles in the presence of trapped lee waves, Tellus, Ser. A, 50, 12-25, 1998.

Lott, F., and M. J. Miller, A new subgrid-scale orographic drag parametrization: Its formulation and testing, Q. J. R. Meteorol. Soc., 123, 101-127, 1997.

Lu, W., and D. C. Fritts, Spectral estimates of gravity wave enrgy and momentum fluxes, III, Gravity wave-tidal interactions, J. Atmos. Sci., 50, 3714-3727, 1993.

Lübken, F.-J., Seasonal variations of turbulent energy dissipation rates at high latitudes as determined by in situ measurements of neutral density fluctuations, J. Geophys. Res., 102, 13,441-13,456, 1997.

Lübken, F.-J., Thermal structure of the Arctic summer mesosphere, J. Geophys. Res., 104, 9135-9149, 1999.

Lübken, F.-J., W. Hillert, G. Lehmacher, and U. von Zahn, Experiments revealing small impact of turbulence on the energy budget of the mesosphere and lower thermosphere, J. Geophys. Res., 98, 20,369-20,384, 1993.

Lübken, F.-J., M. J. Jarvis, and G. O. L. Jones, First in situ temperature measurements at the Antarctic summer mesopause, Geophys. Res. Lett., 26, 3581-3584, 1999.

Luo, Z., and D. C. Fritts, Gravity-wave excitation by geostrophic adjustment of the jet stream, part II, Three-dimensional forcing, J. Atmos. Sci., 50, 105-115, 1993. 
Luo, Z., D. C. Fritts, R. W. Portmann, and G. E. Thomas, Dynamical and radiative forcing of the summer mesopause circulation and thermal structure, 2, Seasonal variations, $J$. Geophys. Res., 100, 3129-3137, 1995.

Maekawa, Y., S. Fukao, and S. Kato, Vertical propagation characteristics of internal gravity waves around the mesopause observed by the Arecibo UHF radar, J. Atmos. Terr. Phys., 49, 73-80, 1987.

Manson, A. H., and C. E. Meek, Gravity wave propagation characteristics $(60-120 \mathrm{~km})$ as determined by the Saskatoon MF radar (Gravnet) system: $1983-85$ at 52N, $107 \mathrm{~W}, J$. Atmos. Sci., 45, 932-946, 1988.

Manson, A. H., and C. E. Meek, Characteristics of gravity waves $(10$ min- 6 hours $)$ at Saskatoon $\left(52^{\circ} \mathrm{N}, 107^{\circ} \mathrm{W}\right)$ : Observations by the phase coherent medium frequency radar, J. Geophys. Res., 98, 20,357-20,367, 1993.

Manson, A. H., C. E. Meek, J. Qian, and C. S. Gardner, Spectra of gravity wave density and wind perturbations observed during Arctic Noctilucent Cloud (ANLC-93) campaign over the Canadian Prairies: Synergistic airborne $\mathrm{Na}$ lidar and MF radar observations, J. Geophys. Res., 103, 6455-6565, 1998a.

Manson, A. H., C. E. Meek, and G. E. Hall, Correlations of gravity waves and tides in the mesosphere over Saskatoon, J. Atmos. Sol. Terr. Phys., 60, 1089-1107, 1998b.

Manzini, E., and K. Hamilton, Middle atmospheric traveling waves forced by latent and convective heating, J. Atmos. Sci., 50, 2180-2200, 1993.

Manzini, E., and N. A. McFarlane, The effect of varying the source spectrum of a gravity wave parameterization in a middle atmosphere general circulation model, J. Geophys. Res., 103, 31,523-31,539, 1998.

Marks, C. J., Some features of the climatology of the middle atmosphere revealed by Nimbus 5 and 6, J. Atmos. Sci., 46, 2485-2508, 1989.

Marks, C. J., and S. D. Eckermann, A three-dimensional nonhydrostatic ray-tracing model for gravity waves: Formulation and preliminary results for the middle atmosphere, $J$. Atmos. Sci., 52, 1959-1984, 1995.

Marsh, A. K. P., N. J. Mitchell, and L. Thomas, Lidar studies of stratospheric gravity-wave spectra, Planet. Space Sci., 39, 1541-1548, 1991.

Mastrantonio, G., F. Einaudi, D. Fua, and D. P. Lalas, Generation of gravity waves by jet streams in the atmosphere, $J$. Atmos. Sci., 33, 1730-1738, 1976.

Matsuno, T., A quasi one-dimensional model of the middle atmosphere circulation interacting with internal gravity waves, J. Meteorol. Soc. Jpn., 60, 215-226, 1982.

Mayr, H. G., J. G. Mengel, C. O. Hines, K. L. Chan, N. F. Arnold, C. A. Reddy, and H. S. Porter, The gravity wave Doppler spread theory applied in a numerical spectral model of the middle atmosphere, 1, Model and global scale seasonal variations, J. Geophys. Res., 102, 26,077-26,091, 1997a.

Mayr, H. G., J. G. Mengel, C. O. Hines, K. L. Chan, N. F. Arnold, C. A. Reddy, and H. S. Porter, The gravity wave Doppler spread theory applied in a numerical spectral model of the middle atmosphere, 2, Equatorial oscillations, J. Geophys. Res., 102, 26,093-26,105, $1997 \mathrm{~b}$.

Mayr, H. G., J. G. Mengel, K. L. Chan, and H. S. Porter, Seasonal variations of the diurnal tide induced by gravity wave filtering, Geophys. Res. Lett., 25, 943-946, 1998.

McComas, C. H., and F. P. Bretherton, Resonant interaction of oceanic internal waves, J. Geophys. Res., 82, 1397-1412, 1977.

McEwan, A. D., Degeneration of resonantly-excited standing internal gravity waves, J. Fluid Mech., 50, 431-448, 1971.

McEwan, A. D., and R. M. Robinson, Parametric instability of internal gravity waves, J. Fluid Mech., 67, 667-687, 1975.
McFarlane, N. A., The effect of orographically excited gravity wave drag on the general circulation of the lower stratosphere and troposphere, J. Atmos. Sci., 44, 1775-1800, 1987.

McIntyre, M. E., On dynamics and transport near the polar mesopause in summer, J. Geophys. Res., 94, 14,617-14,628, 1989.

McLandress, C., On the importance of gravity waves in the middle atmosphere and their parameterization in general circulation models, J. Atmos. Sol. Terr. Phys., 60, 1357-1383, 1998.

McLandress, C., The seasonal variation of the propagating diurnal tide in the mesosphere and lower thermosphere, part I, The role of gravity waves and planetary waves, $J$. Atmos. Sci., 59, 893-906, 2002.

McLandress, C., and W. E. Ward, Tidal/gravity wave interactions and their influence on the large-scale dynamics of the middle atmosphere: Model results, J. Geophys. Res., 99, 8139-8156, 1994.

McLandress, C., M. J. Alexander, and D. L. Wu, Microwave Limb Sounder observations of gravity waves in the stratosphere: A climatology and interpretation, J. Geophys. Res., 105, 11,947-11,967, 2000.

Medvedev, A. S., and G. P. Klaassen, Vertical evolution of gravity wave spectra and the parameterization of associated wave drag, J. Geophys. Res., 100, 25,841-25,853, 1995.

Medvedev, A. S., and G. P. Klaasen, Parameterization of gravity wave momentum deposition based on nonlinear wave interactions: Basic formulation and sensitivity tests, $J$. Atmos. Sol. Terr. Phys., 62, 1015-1033, 2000.

Medvedev, A. S., and G. P. Klaasen, Realistic semiannual oscillation simulated in a middle atmosphere general circulation model, Geophys. Res. Lett., 28, 733-736, 2001.

Medvedev, A. S., G. P. Klaassen, and S. R. Beagley, On the role of an anisotropic gravity wave spectrum in maintaining the circulation of the middle atmosphere, Geophys. Res. Lett., 25, 509-512, 1998.

Meek, C. E., I. M. Reid, and A. H. Manson, Observations of mesospheric wind velocities, 2, Cross sections of power spectral density for 48-8 hours, $8-1$ hours, and 1 hour to 10 min over 60-110 km for 1981, Radio Sci., 20, 1383-1402, 1985.

Meyer, C. K., Gravity wave interactions with the diurnal propagating tide, J. Geophys. Res., 104, 4223-4239, 1999a.

Meyer, C. K., Gravity wave interactions with mesospheric planetary waves: A mechanism for penetration into the thermosphere-ionosphere system, J. Geophys. Res., 104, 28,181-28,196, 1999 b.

Meyer, W., R. Siebenmorgen, and H.-U. Widdel, Estimates of gravity wave momentum fluxes in the winter and summer high mesosphere over northern Scandinavia, J. Atmos. Terr. Phys., 51, 311-319, 1989.

Mied, R. P., The occurrence of parametric instabilities in finite amplitude internal gravity waves, J. Fluid Mech., 78, 763784, 1976.

Miller, M. J., T. N. Palmer, and R. Swinbank, Parameterization and influence of subgridscale orography in general circulation and numerical weather prediction models, $M A P$ Handbk., 40, 84-109, 1989.

Mitchell, N. J., and V. S. C. Howells, Vertical velocities associated with gravity waves measured in the mesosphere and lower thermosphere with the EISCAT VHF radar, Ann. Geophys., 16, 1367-1379, 1998.

Mitchell, N. J., L. Thomas, and A. K. P. Marsh, Lidar observations of long-period gravity waves in the stratosphere, Ann. Geophys., 9, 588-596, 1991.

Mitchell, N. J., L. Thomas, and I. T. Prichard, Gravity waves in the stratosphere and troposphere observed by lidar and MST radar, J. Atmos. Terr. Phys., 56, 939-947, 1994.

Mitchell, N. J., A. J. McDonald, S. J. Reid, and J. D. Price, 
Observations of gravity waves in the upper and lower stratosphere by lidar and ozonesondes, Ann. Geophys., 14, 309314, 1996

Miyahara, S., Suppression of stationary planetary waves by internal gravity waves in the mesosphere, J. Atmos. Sci., 42, $100-107,1985$.

Miyahara, S., and J. M. Forbes, Interactions between gravity waves and the diurnal tide in the mesosphere and lower thermosphere, J. Meteorol. Soc. Jpn., 69, 523-531, 1991.

Miyahara, S., Y. Hayashi, and J. D. Mahlman, Interactions between gravity waves and planetary-scale flow simulated by the GFDL "SKYHI" general circulation model, J. Atmos. Sci., 43, 1844-1861, 1986.

Müller, K. M., U. Langematz, and S. Pawson, The stratopause semiannual oscillation in the Berlin Troposphere-Stratosphere-Mesosphere GCM, J. Atmos. Sci., 54, 2749-2759, 1997.

Müller, P., G. Holloway, F. Henyey, and N. Pomphrey, Nonlinear interactions among internal gravity waves, Rev. Geophys., 24, 493-536, 1986.

Muraoka, Y., T. Sugiyama, K. Kawahira, T. Sato, T. Tsuda, S. Fukao, and S. Kato, Cause of monochromatic inertia-gravity wave breaking observed by the MU radar, Geophys. Res. Lett., 15, 1349-1352, 1988.

Murayama, Y., T. Tsuda, and S. Fukao, Seasonal variation of gravity wave activity in the lower atmosphere observed with the MU radar, J. Geophys. Res., 99, 23,057-23,069, 1994.

Murphy, D. J., and R. A. Vincent, Mesospheric momentum fluxes over Adelaide during the 2-day wave: Results and interpretation, J. Geophys. Res., 103, 28,627-28,636, 1998.

Nakamura, T., T. Tsuda, S. Fukao, and S. Kato, Comparison of the mesospheric gravity waves observed with the MU radar $(35 \mathrm{~N})$ and the Adelaide MF radar (35S), Geophy. Res. Lett., 20, 803-806, 1993a.

Nakamura, T., T. Tsuda, S. Fukao, S. Kato, A. H. Manson, and C. E. Meek, Comparative observations of short-period gravity waves $(10-100 \mathrm{~min})$ in the mesosphere in 1989 by Saskatoon $\mathrm{MF}$ radar $\left(52^{\circ} \mathrm{N}\right)$, Canada and the $\mathrm{MU}$ radar $\left(35^{\circ} \mathrm{N}\right)$, Japan, Radio Sci., 28, 729-746, $1993 \mathrm{~b}$.

Nakamura, T., T. Tsuda, M. Yamamoto, S. Fukao, and S. Kato, Characteristics of gravity waves in the mesosphere observed with the middle and upper atmosphere radar, 1, Momentum flux, J. Geophys. Res., 98, 8899-8910, 1993c.

Nakamura, T., T. Tsuda, M. Yamamoto, S. Fukao, and S. Kato, Characteristics of gravity waves in the mesosphere observed with the middle and upper atmosphere radar, 2, Propagation direction, J. Geophys. Res., 98, 8911-8923, 1993d.

Nakamura, T., T. Tsuda, S. Fukao, A. H. Manson, C. E. Meek, R. A. Vincent, and I. M. Reid, Mesospheric gravity waves at Saskatoon $\left(52^{\circ} \mathrm{N}\right)$, Kyoto $\left(35^{\circ} \mathrm{N}\right)$, and Adelaide $\left(35^{\circ} \mathrm{S}\right), J$. Geophys. Res., 101, 7005-7012, 1996.

Nakamura, T., D. C. Fritts, J. R. Isler, T. Tsuda, and R. A. Vincent, Short-period fluctuations of the diurnal tide observed with low-latitude MF and meteor radars during CADRE: Evidence for gravity wave/tidal interactions, $J$. Geophys. Res., 102, 26,225-26,238, 1997.

Nakamura, T., T. Tsuda, H. Miyagawa, Y. Matsushita, H. Fukunishi, Y. Takahashi, and Y. Yamada, Propagation directions of gravity wave patterns observed in OH CCD images during the SEEK campaign, Geophys. Res. Lett., 25, 1793-1796, 1998.

Nance, L. B., and D. D. Durran, A modeling study of nonstationary trapped mountain lee waves, part II, Nonlinearity, $J$. Atmos. Sci., 55, 1429-1445, 1998.

Nastrom, G. D., and D. C. Fritts, Sources of mesoscale variability of gravity waves, part I, Topographic excitation, $J$. Atmos. Sci., 49, 101-110, 1992.

Nastrom, G. D., and K. S. Gage, A climatology of atmospheric wavenumber spectra of wind and temperature observed by commercial aircraft, J. Atmos. Sci., 42, 950-960, 1985.

Nastrom, G. D., B. B. Balsley, and D. A. Carter, Mean meridional winds in the mid- and high-latitude summer mesosphere, Geophys. Res. Lett., 9, 139-142, 1982.

Nastrom, G. D., D. C. Fritts, and K. S. Gage, An investigation of terrain effects on the mesoscale spectrum of atmospheric motions, J. Atmos. Sci., 44, 3087-3096, 1987.

Nastrom, G. D., T. E. VanZandt, and J. M. Warnock, Vertical wavenumber spectra of wind and temperature from highresolution balloon soundings in the lower atmosphere over Illinois, J. Geophys. Res., 102, 6685-6702, 1997.

Nissen, K. M., P. Braesicke, and U. Langematz, QBO, SAO, and tropical waves in the Berlin TSM GCM: Sensitivity to radiation, vertical resolution, and convection, J. Geophys. Res., 105, 24,771-24,790, 2000.

Norton, W. A., and J. Thuburn, The two-day wave in a middle atmosphere GCM, Geophys. Res. Lett., 23, 2113-2116, 1996.

Norton, W. A., and J. Thuburn, The mesosphere in the extended UGAMP GCM, in Gravity Wave Processes: Their Parameterization in Global Climate Models, NATO ASI Ser., Ser. I, vol. 50, edited by K. Hamilton, pp. 383-401, Springer-Verlag, New York, 1997.

Norton, W. A., and J. Thuburn (Eds.), Sensitivity of mesospheric mean flow, planetary waves, and tides to strength of gravity wave drag, J. Geophys. Res., 104, 30,897-30,911, 1999.

Ogino, S., M. D. Yamanaka, and S. Fukao, Meridional variation of lower stratospheric gravity wave activity: A quick look at Hakuho-Maru J-COARE cruise rawinsonde data, $J$. Meteorol. Soc. Jpn., 73, 407-413, 1995.

O’Sullivan, D., and T. J. Dunkerton, Seasonal development of the extratropical QBO in a numerical model of the middle atmosphere, J. Atmos. Sci., 51, 3706-3721, 1994.

O'Sullivan, D., and T. J. Dunkerton, Generation of inertiagravity waves in a simulated life cycle of baroclinic instability, J. Atmos. Sci., 52, 3695-3716, 1995.

Palmer, T. L., D. C. Fritts, Ø. Andreassen, and I. Lie, Threedimensional evolution of Kelvin-Helmholtz billows in stratified compressible flow, Geophys. Res. Lett., 21, 2287-2290, 1994.

Palmer, T. L., D. C. Fritts, and Ø. Andreassen, Evolution and breakdown of Kelvin-Helmholtz billows in stratified compressible flows, II, Instability structure, evolution, and energetics, J. Atmos. Sci., 53, 3192-3212, 1996.

Palmer, T. N., G. J. Shutts, and R. Swinbank, Alleviation of a systematic westerly bias in general circulation and numerical weather prediction models through an orographic gravity wave drag parameterization, Q. J. R. Meteorol. Soc., 112, 1001-10039, 1986.

Pandya, R. E., and M. J. Alexander, Linear stratospheric gravity waves above convective thermal forcing, J. Atmos. Sci., 56, 2434-2446, 1999.

Pawson, S., Effects of gravity wave drag in the Berlin troposphere-stratosphere-mesosphere GCM, in Gravity Wave Processes: Their Parameterization in Global Climate Models, NATO ASI Ser., Ser. I, vol. 50, edited by K. Hamilton, pp. 327-336, Springer-Verlag, New York, 1997.

Pfister, L., W. Starr, R. Craig, and M. Loewenstein, Small-scale motions observed by aircraft in the tropical lower stratosphere: Evidence for mixing and its relationship to largescale flows, J. Atmos. Sci., 43, 3210-3225, 1986.

Pfister, L., S. Scott, M. Loewenstein, S. Bowen, and M. Legg, Mesoscale disturbances in the tropical stratosphere excited by convection: Observations and effects on the stratospheric momentum budget, J. Atmos. Sci., 50, 1058-1075, 1993a.

Pfister, L., K. R. Chan, T. P. Bui, S. Bowen, M. Legg, B. Gary, K. Kelly, M. Proffitt, and W. Starr, Gravity waves generated by a tropical cyclone during the STEP tropical feild pro- 
gram: A case study, J. Geophys. Res., 98, 8611-8638, 1993 b.

Piani, C., D. Durran, M. J. Alexander, and J. R. Holton, A numerical study of three-dimensional gravity waves triggered by deep tropical convection and their role in the dynamics of the QBO, J. Atmos. Sci., 57, 3689-3702, 2000.

Picard, R. H., R. R. O’Neil, H. A. Gardiner, J. Gibson, J. R. Winick, W. O. Gallery, A. T. Stair Jr., P. P. Wintersteiner, E. R. Hegblom, and E. Richards, Remote sensing of discrete stratospheric gravity-wave structure at 4.3-micron from the MSX satellite, Geophys. Res. Lett., 25, 2809-2812, 1998.

Preusse, P., B. Schaeler, J. T. Bacmeister, and D. Offermann, Evidence for gravity waves in CRISTA temperatures, $A d v$. Space Res., 24, 1601-1604, 1999.

Preusse, P., S. D. Eckermann, and D. Offermann, Comparison of global distributions of zonal-mean gravity wave variance inferred from different satellite instruments, Geophys. Res. Lett., 27, 3877-3880, 2000.

Preusse, P., A. Drnbrack, S. D. Eckermann, M. Riese, B. Schaeler, J. T. Bacmeister, D. Broutman, and K. U. Grossmann, Space-based measurements of stratospheric mountain waves by CRISTA, 1, Sensitivity, analysis method, and a case study, J. Geophys. Res., 107(D23), 8178, doi:10.1029/ 2001JD000699, 2002.

Quinn, E. P., and R. H. Holzworth, Quasi-Lagrangian measurements of density surface fluctuations and power spectra in the stratosphere, J. Geophys. Res., 92, 10,926-10,932, 1987.

Ray, E. A., M. J. Alexander, and J. R. Holton, An analysis of the structure and forcing of the equatorial semiannual oscillation in zonal wind, J. Geophys. Res., 103, 1759-1774, 1998.

Reeder, M. J., and M. Griffiths, Stratospheric inertia-gravity waves generated in a numerical model of frontogenesis, II, Wave sources and generation mechanisms, Q. J. R. Meteorol. Soc., 122, 1175-1195, 1996.

Reid, I. M., and R. A. Vincent, Measurements of mesospheric gravity wave momentum fluxes and mean flow accelerations at Adelaide, Australia, J. Atmos. Terr. Phys., 49, 443-460, 1987.

Reid, I. M., R. Rüster, P. Czechowsky, and G. Schmidt, VHF radar measurements of momentum flux in the summer polar mesosphere over Andenes $\left(69^{\circ} \mathrm{N}, 16^{\circ} \mathrm{E}\right)$, Norway, Geophys. Res. Lett., 15, 1263-1266, 1988.

Ricciardulli, L., and R. R. Garcia, The excitation of equatorial waves by deep convection in the NCAR Community Climate Model (CCM3), J. Atmos. Sci., 57, 3461-3487, 2000.

Riggin, D. M., D. C. Fritts, C. D. Fawcett, E. Kudeki, and M. H. Hitchman, Radar observations of gravity waves over Jicamarca, Peru during the CADRE campaign, J. Geophys. Res., 102, 26,263-26,281, 1997.

Rind, D., R. Suozzo, and N. K. Balachandran, The GISS global climate-middle atmosphere model, part II, Model variability due to interactions between planetary waves, the mean circulation and gravity wave drag, J. Atmos. Sci., 45, 371386, 1988.

Roble, R. G., and E. C. Ridley, A thermosphere-ionospheremesosphere- electrodynamics general circulation model (time-GCM): Equinox solar cycle minimum simulations (30-500 km), Geophys. Res. Lett., 21, 417-420, 1994.

Rocken, C., et al., Analysis and validation of GPS/MET data in the neutral atmosphere, J. Geophys. Res., 102, 29,849-29,866, 1997.

Rosenlof, K. H., Seasonal cycle of the residual mean meridional circulation in the stratosphere, J. Geophys. Res., 100, 5173-5191, 1995.

Rosenlof, K. H., Summer hemisphere differences in temperature and transport in the lower stratosphere, J. Geophys. Res., 101, 19,129-19,136, 1996.
Salby, M. L., and R. R. Garcia, Transient response to localized episodic heating in the tropics, part I, Excitation and shorttime near-field behaviour, J. Atmos. Sci., 44, 458-498, 1987.

Sassi, F., and R. R. Garcia, A one-dimensional model of the semiannual oscillation driven by convectively forced gravity waves, J. Atmos. Sci., 51, 3167-3182, 1994.

Sassi, F., and R. R. Garcia, The role of equatorial waves forced by convection in the tropical semiannual oscillation, $J$. Atmos. Sci., 54, 1925-1942, 1997.

Sassi, F., R. R. Garcia, and B. A. Boville, The stratopause semiannual oscillation in the NCAR Community Climate Model, J. Atmos. Sci., 50, 3608-3624, 1993.

Sato, K., Vertical wind disturbances in the afternoon of midsummer revealed by the MU radar, Geophys. Res. Lett., 19, 1943-1946, 1992.

Sato, K., Small-scale wind disturbances observed by the MU radar during the passage of typhoon Kelly, J. Atmos. Sci., 50, 518-537, 1993.

Sato, K., A statistical study of the structure, saturation and sources of inertio-gravity waves in the lower stratosphere observed with the MU radar, J. Atmos. Terr. Phys., 56, 755-774, 1994.

Sato, K., and T. J. Dunkerton, Estimates of momentum flux associated with equatorial Kelvin and gravity waves, J. Geophys. Res., 102, 26,247-26,261, 1997.

Sato, T., and R. F. Woodman, Fine altitude resolution radar observations of stratospheric turbulent layers by the Arecibo $430 \mathrm{MHz}$ radar, J. Atmos. Sci., 39, 2546-2552, 1982.

Sato, K., and M. Yamada, Vertical structure of atmospheric gravity waves revealed by the wavelet analysis, J. Geophys. Res., 99, 20,623-20,631, 1994.

Sato, K., F. Hasegawa, and I. Hirota, Short-period disturbances in the equatorial lower stratosphere, J. Meteorol. Soc. Jpn., 72, 859-872, 1994.

Sato, K., H. Hashiguchi, and S. Fukao, Gravity waves and turbulence associated with cumulus convection observed with the UHF/VHF clear-air Doppler radars, J. Geophys. Res., 100, 7111-7120, 1995.

Sato, K., D. O'Sullivan, and T. J. Dunkerton, Low-frequency inertia-gravity waves in the stratosphere revealed by threeweek continuous observation with the MU radar, Geophys. Res. Lett., 24, 1739-1742, 1997.

Sato, K., T. Kumakura, and M. Takahashi, Gravity waves appearing in a high-resolution GCM simulation, J. Atmos. Sci., 56, 1005-1018, 1999.

Scaife, A. A., N. Butchart, C. D. Warner, D. Stainforth, and W. Norton, Realistic quasi-biennial osciallations in a simulation of the global climate, Geophys. Res. Lett., 27, 34813484, 2000.

Schar, C., and D. R. Durran, Vortex formation and vortex shedding in continuously stratified flows past isolated topography, J. Atmos. Sci., 54, 534-554, 1997.

Scheffler, A. O., and C. H. Liu, The effects of Doppler shift on the gravity wave spectra observed by MST radar, J. Atmos. Terr. Phys., 48, 1225-1231, 1986.

Schoeberl, M. R., Dynamics of the middle atmosphere, Rev. Geophys., 25, 501-507, 1987.

Scinocca, J. F., and R. Ford, The nonlinear forcing of largescale internal gravity waves by stratified shear instability, $J$. Atmos. Sci., 57, 653-672, 2000.

Scinocca, J. F. and N. A. McFarlane, Anisotropy in the parameterization of drag due to freely propagating gravity waves and low-level dynamics, Q. J. R. Meteorol. Soc., 126, 23532393, 2000.

Senft, D. C., and C. S. Gardner, Seasonal variability of gravity wave activity and spectra in the mesopause region at Urbana, J. Geophys. Res., 96, 17,229-17,264, 1991. 
Shimizu, A., and T. Tsuda, Characteristics of Kelvin waves and gravity waves observed with radiosondes over Indonesia, $J$. Geophys. Res., 102, 26,159-26,171, 1997.

Shine, K., Sources and sinks of zonal momentum in the middle atmosphere diagnosed using the diabatic circulation, Q. J. R. Meteorol. Soc., 115, 265-292, 1989.

Shutts, G. J., Gravity-wave parameterization over complex terrain: The effect of critical level absorption in directional wind shear, Q. J. R. Meteorol. Soc., 121, 1005-1021, 1995.

Shutts, G. J., Stationary gravity-wave structure in flows with directional wind Shear, Q. J. R. Meteorol. Soc., 124, 14211442, 1998.

Shutts, G. J., M. Kitchen, and P. H. Hoare, A large amplitude gravity wave detected by radiosonde, Meteorol. Mag., 117, 306-310, 1988.

Sidi, C., J. Lefrere, F. Dalaudier, and J. Barat, An improved atmospheric buoyancy wave model, J. Geophys. Res., 93, 774-790, 1988.

Siskind, D. E., S. Eckermann, J. McCormack, M. J. Alexander, and J. Bacmeister, Hemispheric differences in the temperature of the summertime stratosphere and mesosphere, $J$. Geophys. Res., 108, doi:10.1029/2002JD002095, in press, 2003.

Smith, A. K., Longitudinal variations in mesospheric winds: Evidence for gravity wave filtering by planetary waves, $J$. Atmos. Sci., 53, 1156-1173, 1996.

Smith, A. K., and G. P. Brasseur, Numerical simulation of the seasonal variation of mesospheric water vapor, J. Geophys. Res., 96, 7553-7563, 1991.

Smith, A. K., and L. V. Lyjak, An observational estimate of gravity wave drag from the momentum balance in the middle atmosphere, J. Geophys. Res., 90, 2233-2241, 1985.

Smith, R. B., A measurement of mountain drag, J. Atmos. Sci., 35, 1644-1654, 1978.

Smith, R. B., On severe downslope winds, J. Atmos. Sci., 42, 2597-2603, 1985.

Smith, S. A., D. C. Fritts, and T. E. VanZandt, Comparison of mesospheric wind spectra with a gravity wave model, Radio Sci., 20, 1331-1338, 1985.

Smith, S. A., D. C. Fritts, and T. E. VanZandt, Evidence for a saturated spectrum of atmospheric gravity waves, J. Atmos. Sci., 44, 1404-1410, 1987.

Smyth, W. D., Dissipation-range geometry and scalar spectra in sheared stratified turbulence, J. Fluid Mech., 401, 209242, 1999.

Smyth, W. D., and J. N. Moum, Anisotropy of turbulence in stably stratified mixing layers, Phys. Fluids, 12, 1343-1362, 2000.

Sonmor, L. J., and G. P. Klaassen, Higher-order resonant instabilities of internal gravity waves, J. Fluid Mech., 324, 1-23, 1996.

Sonmor, L. J., and G. P. Klaassen, Toward a unified theory of gravity wave stability, J. Atmos. Sci., 54, 2655-2680, 1997.

Sonmor, L. J., and G. P. Klaassen, Mechanisms of gravity wave focusing in the middle atmosphere, J. Atmos. Sci., 57, 493510, 2000.

Staquet, C., and J. Sommeria, Internal waves, turbulence and mixing in stratified flows: A report on Euromech Colloquium 339, J. Fluid Mech., 314, 349-371, 1996.

Strobel, D. F., J. P. Apruzese, and M. R. Schoeberl, Energy balance constraints on gravity wave induced eddy diffusion in the mesosphere and lower thermosphere, J. Geophys. Res., 90, 13,067-13,072, 1985.

Strobel, D. F., M. E. Summers, R. M. Bevilacqua, M. T. DeLand, and M. Allen, Vertical constituent transport in the mesosphere, J. Geophys. Res., 92, 6691-6698, 1987.

Sutherland, B. R., Propagation and reflection of internal waves, Phys. Fluids, 11, 1081-1090, 1999.

Sutherland, B. R., Internal wave reflection in uniform shear,
Q. J. R. Meteorol. Soc., 126, 3255-3287, 2000.

Sutherland, B. R., Finite-amplitude internal wavepacket dispersion and breaking, J. Fluid Mech., 429, 343-380, 2001.

Swenson, G. R., and P. J. Espy, Observations of two-dimensional airglow structure and $\mathrm{Na}$ density from the ALOHA, October 9, 1993, "Storm Flight", Geophys. Res. Lett., 22, 2845-2848, 1995.

Swenson, G. R., and S. B. Mende, $\mathrm{OH}$ emission and gravity waves (including a breaking wave) in all-sky imagery from Bear Lake, UT, Geophys. Res. Lett., 21, 2239-2242, 1994.

Swenson, G. R., R. Haque, W. Yang, and C. S. Gardner, Momentum and energy fluxes of monochromatic gravity waves observed by an $\mathrm{OH}$ imager at Starfire Optical Range, New Mexico, J. Geophys. Res., 104, 6067-6080, 1999.

Swenson, G. R., M. J. Alexander, and R. Haque, Dispersion imposed limits on atmospheric gravity waves in the mesosphere: Observations from $\mathrm{OH}$ airglow, Geophys. Res. Lett., 27, 875-878, 2000.

Takahashi, M., Simulation of the stratospheric quasi-biennial oscillation using a general circulation model, Geophys. Res. Lett., 23, 661-664, 1996.

Takahashi, M., The first realistic simulation of the stratosphereic quasi-biennial oscillation in a general circulation model, Geophys. Res. Lett., 26, 1307-1310, 1999.

Takahashi, M., and B. A. Boville, A three-dimensional simulation of the equatorial quasi-biennial oscillation, J. Atmos. Sci., 49, 1020-1035, 1992.

Takahashi, M., and J. R. Holton, The mean zonal flow response to Rossby wave and gravity wave forcing in the equatorial lower stratosphere: Relationship to the QBO, $J$. Atmos. Sci., 48, 2078-2087, 1991.

Taylor, M. J., and F. J. Garcia, A two-dimensional spectral analysis of short period gravity waves imaged in the $\mathrm{OI}(557.7 \mathrm{~nm})$ and near infrared $\mathrm{OH}$ nightglow emissions over Arecibo, Puerto Rico, Geophys. Res. Lett., 22, 24732476, 1995.

Taylor, M. J., P. J. Espy, D. J. Baker, R. J. Sica, P. C. Neal, and W. R. Pendleton Jr., Simultaneous intensity, temperature and imaging measurements of short period wave structure in the OH nightglow emission, Planet. Space Sci., 39, 11711188, 1991.

Taylor, M. J., Y. Y. Gu, X. Tao, C. S. Gardner, and M. B. Bishop, An investigation of intrinsic gravity wave signatures using coordinated lidar and nightglow image measurements, Geophys. Res. Lett., 22, 2853-2856, 1995.

Taylor, M. J., W. R. Pendleton Jr., S. Clark, H. Takahashi, D. Gobbi, and R. A. Goldberg, Image measurements of shortperiod gravity waves at equatorial latitudes, J. Geophys. Res., 102, 26,283-26,299, 1997.

Taylor, M. J., S. H. Seo, T. Nakamura, T. Tsuda, H. Fukunishi, and Y. Takahashi, Long base-line measurements of shortperiod mesospheric gravity waves during the SEEK campaign, Geophys. Res. Lett., 25, 1797-1800, 1998.

Thayaparan, T., W. K. Hocking, and J. MacDougall, Observational evidence of tidal/gravity wave interactions using the UWO 2 MHz radar, Geophys. Res. Lett., 22, 373-376, 1995.

Thomas, L., I. T. Prichard, and I. Astin, Radar observations of an inertia-gravity wave in the troposphere and lower stratosphere, Ann. Geophys., 10, 690-697, 1992.

Thompson, R. O. R. Y., Observations of inertial gravity waves in the stratosphere, Q. J. R. Meteorol. Soc., 104, 691-698, 1978.

Thorpe, S. A., Transitional phenomena and the development of turbulence in stratified fluids: A review, J. Geophys. Res., 92, 5231-5248, 1987.

Thorpe, S. A., Observations of parametric instability and breaking waves in an oscillating tilted tube, J. Fluid Mech., 261, 33-45, 1994. 
Tsuda, T., T. Inoue, D. C. Fritts, T. E. VanZandt, S. Kato, T. Sato, and S. Fukao, MST radar observations of a saturated gravity wave spectrum, J. Atmos. Sci., 46, 2440-2447, 1989.

Tsuda, T., S. Kato, T. Yokoi, T. Inoue, M. Yamamoto, T. E. VanZandt, S. Fukao, and T. Sato, Gravity waves in the mesosphere observed with the middle and upper atmosphere radar, Radio Sci., 25, 1005-1018, 1990a.

Tsuda, T., Y. Murayama, M. Yamamoto, S. Kato, and S. Fukao, Seasonal variation of momentum flux in the mesosphere observed with the MU radar, Geophys. Res. Lett., 17, 725-728, 1990b.

Tsuda, T., T. E. VanZandt, M. Mizumoto, S. Kato, and S. Fukao, Spectral analysis of temperature and Brunt-Vaisala frequency fluctuations observed by radiosondes, J. Geophys. Res., 96, 17,265-17,278, 1991.

Tsuda, T., Y. Murayama, K.-I. Oyama, F. J. Schmidlin, M. Bittner, H. Kanzawa, T. Nakamura, M. D. Yamanaka, S. Fukao, and S. Kato, Rocketsonde observations of the middle atmosphere dynamics at Uchinoura (31N, 131E) during the DYANA campaign, part II, Characteristics of gravity waves, J. Geomag. Geoelectr., 44, 1009-1023, 1992.

Tsuda, T., Y. Murayama, H. Wiryosumarto, S. W. B. Harijono, and S. Kato, Radiosonde obserations of equatorial atmosphere dynamics over Indonesia, 2, characteristics of gravity waves, J. Geophys. Res., 99, 10,507-10,516, 1994a.

Tsuda, T., Y. Murayama, T. Nakamura, R. A. Vincent, A. H. Manson, C. E. Meek, and R. L. Wilson, Variations of the gravity wave characteristics with height, season, and latitude revealed by comparative observations, J. Atmos. Terr. Phys., 56, 555-568, 1994 b.

Tsuda, T., M. Nishida, and C. Rocken, A global morphology of gravity wave activity in the stratosphere revealed by the GPS occultation data (GPS/MET), J. Geophys. Res., 105, 7257-7274, 2000.

Vadas, S. L., and D. C. Fritts, Gravity wave radiation and mean responses to local body forces in the atmosphere, J. Atmos. Sci., 58, 2249-2279, 2001.

Vanneste, J., The instability of internal gravity waves to localized disturbances, Ann. Geophys., 13, 196-210, 1995.

VanZandt, T. E., A universal spectrum of buoyancy waves in the atmosphere, Geophys. Res. Lett., 9, 575-578, 1982.

VanZandt, T. E., and D. C. Fritts, A theory of enhanced saturation of the gravity wave spectrum due to increases in atmospheric stability, Pure Appl. Geophys., 130, 399-420, 1989.

VanZandt, T. E., G. D. Nastrom, and J. L. Green, Frequency spectra of vertical velocity from flatland VHF radar data, $J$. Geophys. Res., 96, 2845-2855, 1991.

Vincent, R. A., MF/HF radar measurements of the dynamics of the mesopause region-A review, J. Atmos. Terr. Phys., 46, 961-974, 1984a.

Vincent, R. A., Gravity wave motions in the mesosphere, $J$. Atmos. Terr. Phys., 46, 119-128, 1984b.

Vincent, R. A., and M. J. Alexander, Gravity waves in the tropical lower stratosphere: An observational study of seasonal and interannual variability, J. Geophys. Res., 105, 17,971-17,982, 2000.

Vincent, R. A., and S. D. Eckermann, VHF radar observations of mesoscale motions in the troposphere: Evidence for gravity wave Doppler shifting, Radio Sci., 25, 1019-1037, 1990.

Vincent, R. A., and D. C. Fritts, A climatology of gravity waves in the mesosphere and lower thermosphere over Adelaide, Australia, J. Atmos. Sci., 44, 748-760, 1987.

Vincent, R. A., and I. M. Reid, HF Doppler measurements of mesospheric momentum fluxes, J. Atmos. Sci., 40, 13211333, 1983.

Vincent, R. A., S. J. Allen, and S. D. Eckermann, Gravity-wave parameters in the lower stratosphere, in Gravity Wave Pro- cesses: Their Parameterization in Global Climate Models, NATO ASI Ser., Ser. I, vol. 50, edited by K. Hamilton, pp. 7-25, Springer-Verlag, New York, 1997.

Wada, K., T. Nitta, and K. Sato, Equatorial inertia-gravity waves in the lower stratosphere revealed by TOGACOARE IOP data, J. Meteorol. Soc. Jpn., 77, 721-736, 1999.

Walterscheid, R. L., Inertio-gravity wave induced accelerations of mean flow having an imposed periodic component: Implications for tidal observations in the meteor region, $J$. Geophys. Res., 86, 9698-9706, 1981.

Walterscheid, R. L., Propagation of small-scale gravity waves through larg-scale internal wave fields: Eikonal effects at low-frequency approximation critical levels, J. Geophys. Res., 105, 18,027-18,037, 2000.

Walterscheid, R. L., J. H. Hecht, R. A. Vincent, I. M. Reid, J. Woithe, and M. J. Hickey, Analysis and interpretation of airglow and radar observations of quasi-monochromatic gravity waves in the upper mesosphere and lower thermosphere over Adelaide, Australia $\left(35^{\circ} \mathrm{S}, 138^{\circ} \mathrm{E}\right), \mathrm{J}$. Atmos. Sol. Terr. Phys., 61, 461-478, 1999.

Wang, D.-Y., and D. C. Fritts, Mesospheric momentum fluxes observed by the MST Radar at Poker Flat, Alaska, J. Atmos. Sci., 47, 1511-1521, 1990.

Wang, D.-Y., and D. C. Fritts, Evidence of gravity wave-tidal interaction observed near the summer mesopause at Poker Flat, Alaska, J. Atmos. Sci., 48, 572-583, 1991.

Warner, C. D., and M. E. McIntyre, On the propagation and dissipation of gravity wave spectra through a realistic middle atmosphere, J. Atmos. Sci., 53, 3213-3235, 1996.

Warner, C. D., and M. E. McIntyre, An ultra-simple spectral parameterization for non-orographic gravity waves, J. Atmos. Sci., 58, 1837-1857, 2001.

Weinstock, J., Nonlinear theory of acoustic-gravity waves, 1, Saturation and enhanced diffusion, J. Geophys. Res., 81, 633-652, 1976.

Weinstock, J., Nonlinear theory of gravity waves: Momentum deposition, generalized Rayleigh friction, and diffusion, $J$. Atmos. Sci., 39, 1698-1710, 1982.

Weinstock, J., Simplified derivation of an algorithm for nonlinear gravity waves, J. Geophys. Res., 89, 345-350, 1984.

Weinstock, J., Theoretical gravity wave spectra in the atmosphere: Strong and weak interactions, Radio Sci., 20, 12951300, 1985.

Weinstock, J., Saturated and unsaturated spectra of gravity waves and scale-dependent diffusion, J. Atmos. Sci., 47, 2211-2225, 1990.

Werne, J., and D. C. Fritts, Stratified shear turbulence: Evolution and statistics, Geophys. Res. Lett., 26, 439-442, 1999.

Werne, J. A., and D. C. Fritts, Turbulence and mixing in a stratified shear layer: 3D K-H simulations at $R e=24,000$, Phys. Chem. Earth, 26, 263-268, 2001.

Whiteway, J. A., and A. I. Carswell, Lidar observations of gravity wave activity in the upper stratosphere over Toronto, J. Geophys. Res., 100, 14,113-14,124, 1995.

Whiteway, J. A., and T. J. Duck, Evidence for critical level filtering of atmospheric gravity waves, Geophys. Res. Lett., 23, 145-148, 1996.

Whiteway, J. A., T. J. Duck, D. P. Donovan, J. C. Bird, S. R. Pal, and A. I. Carswell, Measurements of gravity wave activity within and around the Arctic stratospheric vortex, Geophys. Res. Lett., 24, 1387-1390, 1997.

Whitham, G. B., A general approach to linear and nonlinear dispersive waves using a Lagrangian, J. Fluid Mech., 22, 273-283, 1965.

Whitham, G. B., Linear and Nonlinear Waves, John Wiley, New York, 1974.

Williams, B. P., M. A. White, D. A. Krueger, and C. Y. She, Observation of a large amplitude wave and inversion layer leading to convective instability in the mesopause region 
over Fort Collins, $\mathrm{CO}\left(41^{\circ} \mathrm{N}, 105^{\circ} \mathrm{W}\right)$, Geophys. Res. Lett., 29(17), 1850, doi:10.1029/2001GL014514, 2002.

Wilson, R., M.-L. Chanin, and A. Hauchecorne, Gravity waves in the middle atmosphere observed by Rayleigh lidar, 1, Case studies, J. Geophys. Res., 96, 5153-5167, 1991a.

Wilson, R., M.-L. Chanin, and A. Hauchecorne, Gravity waves in the middle atmosphere observed by Rayleigh lidar, part 2, Climatology, J. Geophys. Res., 96, 5169-5183, 1991b.

Winters, K. B., and E. A. D Asaro, 3D wave breaking near a critical level, J. Fluid Mech., 272, 255-284, 1994.

Winters, K. B, and J. J. Riley, Instability of internal waves near a critical level, Dyn. Atmos. Oceans, 16, 249-278, 1992.

Woodman, R. F., B. B. Balsley, F. Aquino, L. Flores, E. Vazquez, M. Sarango, M. M. Huaman, and H. Soldi, First observations of polar summer mesosphere echoes in Antarctic, J. Geophys. Res., 104, 22,577-22,590, 1999.

Worthington, R. M., and L. Thomas, Radar measurements of critical-layer absorption in mountain waves, Q. J. R. Meteorol. Soc., 122, 1263-1282, 1996.

Wu, D. L., and J. W. Waters, Satellite observations of atmospheric variances: A possible indication of gravity waves, Geophys. Res. Lett., 23, 3631-3634, 1996a.

Wu, D. L., and J. W. Waters, Gravity-wave-scale temperature fluctuations seen by the UARS MLS, Geophys. Res. Lett., 23, 3289-3292, 1996b.

Wu, D. L., and J. W. Waters, Observations of gravity waves with the UARS Microwave Limb Sounder, in Gravity Processes: Their Parameterization in Global Climate Models, NATO ASI Ser., Ser. I, vol. 50, edited by K. Hamilton, pp. 103-120, Springer-Verlag, New York, 1997.

Wu, Y.-F., and H.-U. Widdel, Observational evidence of a saturated gravity wave spectrum in the mesosphere, $J$. Atmos. Terr. Phys., 51, 991-996, 1989.

Wu, Y.-F., and H.-U. Widdel, Spectral analysis of atmospheric velocity fluctuations in the mesosphere, J. Atmos. Terr. Phys., 52, 23-33, 1990.

Wu, Y.-F., and H.-U. Widdel, Further study of a saturated gravity wave spectrum in the mesosphere, J. Geophys. Res., 96, 9263-9272, 1991.

Yamada, Y., H. Fukunishi, T. Nakamura, and T. Tsuda, Breakdown of small-scale quasi-stationary gravity wave and transition to turbulence observed in $\mathrm{OH}$ airglow, Geophys. Res. Lett., 28, 2153-2156, 2001.
Yamamoto, M., T. Tsuda, S. Kato, T. Sato, and S. Fukao, A saturated inertia gravity wave in the mesosphere observed by the middle and upper atmosphere radar, J. Geophys. Res., 92, 11,993-11,999, 1987.

Yeh, K. C., and B. Dong, The nonlinear interaction of a gravity wave with the vortical modes, J. Atmos. Terr. Phys., 51, 45-50, 1989.

Yeh, K. C., and C. H. Liu, The instability of atmospheric gravity waves through wave-wave interactions, J. Geophys. Res., 86, 9722-9728, 1981.

Yoshiki, M., and K. Sato, A statistical study of gravity waves in the polar regions based on operational radiosonde data, $J$. Geophys. Res., 105, 17,995-18,011, 2000.

Yuan, L., and D. C. Fritts, Influence of a mean shear on the dynamical instability of an inertio-gravity wave, J. Atmos. Sci., 46, 2562-2568, 1989.

Zhong, L., L. J. Sonmor, A. H. Manson, and C. E. Meek, The influence of time-dependent wind on gravity-wave propagation in the middle atmosphere, Ann. Geophys., 13, 375394, 1995.

Zhu, X., A new theory of saturated gravity wave spectrum in the middle atmosphere, J. Atmos. Sci., 51, 3615-3626, 1994.

Zhu, X., and J. R. Holton, Mean fields induced by local gravity-wave forcing in the middle atmosphere, J. Atmos. Sci., 44, 620-630, 1987.

Zhu, X., P. K. Swaminathan, J.-H. Yee, D. F. Strobel, and D. Anderson, A globally balanced two-dimensional middle atmosphere model: dynamical studies of mesopause meridional circulation and stratosphere-mesosphere exchange, $J$. Geophys. Res., 102, 13,095-13,112, 1997.

Zink, F., and R. A. Vincent, Wavelet analysis of stratospheric gravity wave packets over Macquarie Island, 1, Wave parameters, J. Geophys. Res., 106, 10,275-10,288, 2001 a.

Zink, F., and R. A. Vincent, Wavelet analysis of stratospheric gravity wave packets over Macquarie Island, 2, Intermittency and mean-flow accelerations, J. Geophys. Res., 106, 10,289-10,297, 2001b.

M. J. Alexander and D. C. Fritts, Colorado Research Associates Division, Northwest Research Associates, 3380 Mitchell Lane, Boulder, CO 80301, USA. (dave@co-ra.com) 